\title{
Catalogue of the Cephalopoda Specimens in the Zoology Department of Tokyo University Museum
}

\author{
Ian G. GLEADALL ${ }^{1, *}$ and M. Alejandro SALCEDO-VARGAS ${ }^{2}$ \\ Department of Zoology, University Museum, University of Tokyo, \\ 7-3-1 Hongo, Bunkyo-ku, Tokyo 113-0033, Japan
}

\author{
${ }^{1}$ Graduate School of Information Sciences, Tohoku University, Sendai 980-8579, Japan \\ ${ }^{2}$ Departamento de Biologia, Facultad de Ciencias, Universidad Nacional Autonoma de Mexico, \\ Apartado postal 70-371, Mexico 04510, D.F. Mexico \\ E-mail: asalcedo@netmex.com
}

Received August 28, 2003; final version accepted September 3, 2004

\begin{abstract}
A catalogue of the extant specimens of recent Cephalopoda in the Museum of Tokyo University is here presented including 50 lots of recently designated type material, mostly from species described by Sasaki. Also identified are the specimens of many other species described by Sasaki in his monograph of 1929. An Appendix provides an English summary of a largely ignored nineteenth century list of the oldest specimens in the ZUMT collection, published in Japanese, for comparison with the present collection.
\end{abstract}

\section{Introduction}

A project has been completed recently to curate and identify the Cephalopoda specimens deposited in the Zoology Department of Tokyo University Museum (ZUMT). One of the main objectives was to find the type specimens of Madoka Sasaki (reported recently by Gleadall, 2003a). The present catalogue provides identifications for the entire collection, much of which was used by Sasaki in compiling his monograph on the Cephalopoda (Sasaki, 1929). These specimens were contributed by Aoki, Dean, Hara, Hibino, Iizuka, Ijima, Ikeda, Ishida, Ishikawa, Mitsukuri, Nomura, Okada, Oshima, Owston, Sakamoto, Sasaki, Shimizu, Tago, Takahashi, Terasaki, Tsuchida, Uchida, Wakiya and Watase. Many of these people were involved in collecting or teaching at Tokyo Science College (the forerunner of Tokyo University Faculty of Science) and the Marine Research Station at Misaki (Isono, 1988).

The specimens formerly belonged to the Zoology Department of Tokyo Imperial University (TIU), as it was known prior to World War II. Most were in formalin in their original containers stored in the museum basement and clearly had not been opened since Sasaki's era. The museum building was modernized in 1995 and the specimens are now preserved in $75 \%$ ethanol in a new spirit store on the fourth floor. The types are stored separately in a locked room and the specimen register is available in the Zoology research office nearby.

The preserved specimens of Cephalopoda collected in the early days of TIU were reported in a series of short articles towards the end of the nineteenth century (Ikeda, 1890a, $b, 1891 a-f$ ). However, Ikeda's account seems to have been largely forgotten and was only mentioned in part even in Sasaki's comprehensive monograph. The lots listed by Ikeda are here summarized in an Appendix (see also comments in the main catalogue).

Included in the present catalogue are three lots of specimens to which manuscript names have been assigned but not published. They are here listed as unidentified octopod 'species 1' (sp. 1) and 'sp. 2.' The names probably were not published because of the state of immaturity of the specimens: there are no obvious features to distinguish them from other octopus species. A recent mention of one of the names (Okutani, 2002: 77) does not constitute publication of a new species name according to the International Code of Zoological Nomenclature (ICZN, 1999, Art. 16).

It is clear from the following catalogue that Sasaki numbered his specimens, probably in sequential lots as he inspected them. These numbers (usually prefixed ' $\mathrm{Sa}$ ') appear on the labels of specimens in both the Hokkaido University Fisheries Museum, Hakodate (HUFM), and ZUMT. Attempts to trace Sasaki's family and original notes have been unsuccessful. Two other sets of numerical identification now accompany the specimens: register codes used during the present study (based on the date of inspection); and a three-digit code that is part of a database of all the specimens in the ZUMT collections (presently incomplete for the Cephalopoda at the time of going to press and not yet recorded on any permanent labels with the specimens).

The identifications in the catalogue presented here include some newly revised genus designations, such as Loliolus, Heterololigo and Uroteuthis for species formerly identified as Loligo (based on Anderson, 2000); and Amphioctopus for

\footnotetext{
* Present address and address for correspondence: Tohoku Bunka Gakuen University, 6-45-1 Kunimi, Sendai 981-8551, Japan.

E-mail: octopus@pm.tbgu.ac.jp
} 
some species formerly identified as Octopus (based on Gleadall, 2002, 2003a, b). In some instances, species generally regarded as synonyms have been listed separately (e.g., Sepia andreana and S. sasakii; and Uroteuthis edulis and U. kensaki) to emphasize the type specimens that have recently become accessible for the first time since the original descriptions (Gleadall, 2003a). The dorsal mantle length is recorded for all specimens to facilitate their recognition in comparison with earlier publications on this collection (Ikeda, 1890a, b, 1891 $a-f$; Ijima and Ikeda, 1895a, $b$, 1902; Sasaki, 1909, 1910, 1913a-c, 1914a, b, 1915, 1916, 1917a, b, 1920, 1929; Wakiya and Ishikawa, 1921).

\section{Dates of collection and receipt: Opisthoteuthis depressa}

It can be demonstrated that some of the specimen labels bear the date of receipt by the researcher who later published the specimen identification, rather than the actual date of collection of the specimens. This means that where published dates and the dates on labels are fairly close but do not match exactly, it is inappropriate to exclude such specimens extant in the collection from consideration as type material. The two specimens of Opisthoteuthis depressa are a good example about which there is little confusion. Each has labels mentioning both April and May. With specimen 996.5.45, one label states '22 April, 1909', and another states (in Japanese) 'received May 24.' The holotype (996.5.46) bears a label with the type locality ('Okinose') and the collector's name ('Kuma') and is dated May, 1895. In the original description, Ijima and Ikeda (1895a: 211) gave the collection date as the end of April; but in their later paper the same year (Ijima and Ikeda, 1895b: 323) they mention only that it was brought to them in May, as stated on the only remaining label. This holotype was previously reported to be no longer extant ( $c f$. Sweeney and Roper, 1998: 577; Sweeney in Vecchione et al., 2002: 85).

\section{Sepia longipes}

The syntype of Sepia longipes has with it a label stating that it was purchased by Mr. Shimizu in April, 1906. However, the original publication states that 'it is recorded that Prof. Watase purchased it in August, 1906' (Sasaki, 1913a), and subsequently Sasaki (1914a) gives the exact date as the 18th of August, 1906. The extant ZUMT specimen is closely similar in dorsal mantle length to the largest of the six syntypes and it is the only specimen in the collection from the locality Choshi, Chiba Pref. It is presumed that Shimizu purchased the specimens in April, 1906, and sold them on to Prof. Watase (of TIU) in August the same year.

\section{Moroteuthis loennbergii}

Sasaki (1929) mentioned inspecting seven specimens of Moroteuthis loennbergii, of which it is presumed that five were the original syntypes as described by Ishikawa and Wakiya (1914). Of the three specimens found in the ZUMT collection, one is probably a recent specimen but it could not be confirmed that the remaining specimens are syntypes (although they are from the type locality). The original description provided a large number of measurements of the specimens but the morphology of this species and the soft condition of the specimens are such that the accuracy of measurement possible is too low to enable favourable comparisons. Measurements of specimen 996.5.61 show some resemblance to those of specimen no. 3 of Ishikawa and Wakiya (1914) but specimen 996.5.61A seems quite different from any of the syntypes. It is concluded that these two older specimens are the two non-type specimens mentioned by Sasaki.

\section{Acknowledgements}

We thank Dr. Kazuo Sakamoto, Dr. Ryôichi Arai, Dr. Rei Ueshima and the late Dr. Tokiharu Abe of ZUMT for their support and encouragement, and for access to the Cephalopoda collection. This work was supported in part by a grant to IGG from the Fujiwara Natural History Foundation.

\section{Annotated Catalogue of the Cephalopoda Specimens in the Zoology Department of Tokyo University Museum}

Listed alphabetically within major groups. Reg. No.: Code entered in the Cephalopoda register, with any type material listed first (numbers in square brackets are those assigned during an ongoing computerization of the specimen catalogues at ZUMT: currently there are no permanent labels bearing these numbers). Specimens: figures in parenthesis are dorsal mantle length (DML) measurements in mm. Collection data: Loc., locality (Japan, Honshu, Pacific coast, unless stated otherwise); clarification/modern names in square brackets; Pref. Prefecture. Coll., collected/collector: dates have mostly been converted from the Japanese Meiji, Taisho or Showa years (given in parentheses as M $x$, Tx or $\mathrm{S} x$, respectively) to the corresponding Gregorian year; Collector 'Kuma' is the nickname of Kumakichi Aoki (collector for the Misaki Marine Experimental Station; Isono, 1988); square brackets denote corrections of or additions to information on labels or in original publications. Comments: Type material indicated in bold type; Sa, Sasaki's personal registration numbers included on some labels (corresponding notes of Sasaki have not been found); verbatim comments and alternate identifications in quotes are from original specimen labels (those in parentheses are from temporary labels left with the specimens by T. Okutani in the summer of 2002). Other abbreviations: ALO, descending order of arm lengths. 


\section{Octobrachia: Octopoda: Cirrata}

Opisthoteuthis depressa Ijima and Ikeda, 1895.

\begin{tabular}{|c|c|c|c|}
\hline Reg. No. & Specimen(s) & Collection data & Comments \\
\hline $\begin{array}{l}996.5 .46 \\
{[241]}\end{array}$ & $\begin{array}{l}1 \text { male }(19 \mathrm{~mm} \\
\text { across eyes; eye } \\
\text { diam. } 9 \mathrm{~mm}) .\end{array}$ & $\begin{array}{l}\text { Loc. [Sagami Bay], Okinose, } \\
250 \mathrm{fm}\left[457 \mathrm{~m} \text { ]. Coll. 'May' [April }{ }^{1} \text { ], }\right. \\
\text { 1895, Kuma. }\end{array}$ & $\begin{array}{l}\text { Holotype by monotypy. A small, } \\
\text { thoroughly dissected specimen. Label 'May, 1895' } \\
\text { records date of receipt by Ijima and Ikeda.' }\end{array}$ \\
\hline $\begin{array}{l}996.5 .45 \\
{[008]}\end{array}$ & $\begin{array}{l}1 \text { ?female }(43 \mathrm{~mm} \\
\text { across eyes; eye } \\
\text { diam. } 16 \mathrm{~mm}) .\end{array}$ & $\begin{array}{l}\text { Loc. Yodomi, Sagami Sea. } \\
\text { Coll. 22.iv.1909, Alan Owston. } \\
\text { Yokohama: Owston Collection. }\end{array}$ & 'Recd. 24 May.' $c f$. Pl. VII fig. 7 in Sasaki (1929). \\
\hline
\end{tabular}

${ }^{1}$ Ijima and Ikeda, 1895a: 211, text figs. 1-3. ${ }^{2}$ Ijima and Ikeda, 1895b: 323, Pl. XXXIII figs. 1-9. See also Sasaki, 1929: 10, text fig. 4, Pl. VII figs. $7,8 \mathrm{a}, \mathrm{b}, 9$.

\section{Octopoda: Incirrata}

Haliphron pacificum (Ijima in Ijima and Ikeda, 1902) (originally as Alloposus pacificus).

\begin{tabular}{|c|c|c|c|}
\hline Reg. No. & Specimen(s) & Collection data & Comments \\
\hline $\begin{array}{l}996.5 .48 \\
{[231]}\end{array}$ & 1 spec. (22) & $\begin{array}{l}\text { Loc. Miura [Peninsula, Kanagawa } \\
\text { Pref., Koajiro Bay], Mito, Hasse } \\
\text { Village. Coll. 29.iii.1902 (M 35). }\end{array}$ & $\begin{array}{l}\text { Syntype. } c f . \text { specimen of total length } 54 \mathrm{~mm} \text {. } \\
\text { 'Alloposus pacificus Ij. n. sp. M.S.' Illustr. Sasaki, } \\
\text { 1929, Pl. VIII fig. 6. ('Haliphron atlanticus'*). }\end{array}$ \\
\hline $\begin{array}{l}996.5 .49 \\
{[232]}\end{array}$ & 1 spec. (35) & $\begin{array}{l}\text { Loc. Dokesoba [Sagami Sea, off } \\
\text { Misaki]. Coll. 20.vi.1910, 'hooked up' by K. Aoki. }\end{array}$ & $\begin{array}{l}\text { ?Syntype. } c f \text {. specimen of total length } 114 \mathrm{~mm} \text { (see } \\
\text { Gleadall, 2003a: } 376 \text { ). }\end{array}$ \\
\hline
\end{tabular}

Ref. Ijima and Ikeda, 1902: 87 (footnote by Ijima); see also Sasaki, 1929: 18, text fig. 5 (labelled Alloposus pelagicus in error), Plate VIII figs. 6-8. *cf. identification of Norman et al. 1997: 375 (based on an immature female Haliphron atlanticum of $73 \mathrm{~mm}$ mantle length reported from Indonesian waters; identified with reference to Thore, 1949; $c f$. also Alvarino and Hunter, 1981).

Amphitretus pelagicus Hoyle, 1885.

\begin{tabular}{lccc}
\hline Reg. No. & Specimen(s) & Collection data & \multicolumn{1}{c}{ Comments } \\
\hline 996.5.47 & 1 specimen (93) & (no data) & Poor condition: in several pieces; most internal \\
{$[230]$} & & organs missing. See text. cf. Sasaki, 1929, Pl. VIII \\
& & fig. 5. ('Alloposus pacificus'). \\
\hline
\end{tabular}

Argonauta argo Linnaeus, 1758.

\begin{tabular}{lccc}
\hline Reg. No. & Specimen(s) & Collection data & Comments \\
\hline $\mathbf{9 9 6 . C O - 4 6}$ & 1 female $(23)$ & (no data) & Shell missing. \\
{$[\mathbf{0 0 4}]$} & & & \\
\hline
\end{tabular}

Argonauta hians Lightfoot, 1786.

\begin{tabular}{lccl}
\hline Reg. No. & Specimen $(\mathrm{s})$ & Collection data & \multicolumn{1}{c}{ Comments } \\
\hline $\mathbf{9 9 4 . C O - 0 2}$ & 3 females $(23,9,7)$ & (no data) & Part of egg mass in separate jar. Shell of large female \\
{$[\mathbf{0 3 3}]$} & & is intact $(50 \mathrm{~mm}$ max. diam.). \\
\hline
\end{tabular}

Ocythoe tuberculata Rafinesque, 1814.

\begin{tabular}{|c|c|c|c|}
\hline Reg. No. & Specimen(s) & Collection data & Comments \\
\hline $\begin{array}{l}\text { 994.CO-01 } \\
{[032]}\end{array}$ & 2 specs. $(400,200)$ & $\begin{array}{l}\text { Loc. Awa [Chiba Pref.?], } \\
\text { Kashiwazaki. Coll. Nov., } 1885 .\end{array}$ & $\begin{array}{l}c f . \text { locs. of Sasaki 1929: 28. } c f . \text { lot. \#2 of Ikeda, 1891a: } \\
26 \text { (giving date as Sept., 1885). }\end{array}$ \\
\hline $\begin{array}{l}\text { 996.CO-40 } \\
{[010]}\end{array}$ & 1 female (135) & $\begin{array}{l}\text { Loc. not given. Coll. 27.vii.1911, } \\
\text { S. Hibino. }\end{array}$ & 'ibodako.' \\
\hline $\begin{array}{l}\text { 996.CO-41 } \\
{[011]}\end{array}$ & 1 spec. (95) & (no data) & cf. measurements of Sasaki, 1929: 27. \\
\hline $\begin{array}{l}996 . \mathrm{CO}-42 \\
{[012]}\end{array}$ & 1 spec. $(81)$ & $\begin{array}{l}\text { Loc. [Kanagawa Pref.], Misaki. } \\
\text { Coll. 2.v.1898 (M31), Kuma. }\end{array}$ & cf. measurements of Sasaki, 1929: 27. \\
\hline $\begin{array}{l}\text { 996.CO-47 } \\
{[096]}\end{array}$ & $\begin{array}{l}2 \text { ?females } \\
(143,103)\end{array}$ & $\begin{array}{l}\text { Loc. Boshu [Chiba Pref.], Fuku-ura. } \\
\text { Coll. 7.xi.1883. }\end{array}$ & $\begin{array}{l}\text { Several labels in good condition (good examples for } \\
\text { records of handwriting) } c f \text {. lot. \#1 of Ikeda, 1891a: } 26 .\end{array}$ \\
\hline
\end{tabular}

Ref. Sasaki, 1929: 26, text fig. 8, Pl. III figs. 13, 14, Pl. VIII figs. 12-16. 
Tremoctopus violaceus gracilis (Eydoux and Souleyet, 1852).

\begin{tabular}{|c|c|c|c|}
\hline Reg. No. & Specimen(s) & Collection data & Comments \\
\hline $\begin{array}{l}996.5 .59 \\
{[168]}\end{array}$ & 1 female $(120)$ & $\begin{array}{l}\text { Loc. [Japan Sea], Tango Kuni [Kyoto Pref.], } \\
\text { Tsukino-gun, Taiza [Tango] Village. } \\
\text { Coll. 30.v.1903, Ushikubo. }\end{array}$ & Sa 126. cf. locs. of Sasaki, 1929: 32. \\
\hline $\begin{array}{l}\text { 996.CO-43 } \\
{[013]}\end{array}$ & 1 female (129) & Loc. [Kyushu], Kagoshima. Coll. Aug. 1903 (M36). & - \\
\hline $\begin{array}{l}\text { 996.CO-44 } \\
{[002]}\end{array}$ & 1 female $(83)$ & Coll. 29.ix.1900. & - \\
\hline $\begin{array}{l}002.12 .4 .1 \\
{[109]}\end{array}$ & 1 female $(26)$ & Coll. April, 1902 (M35). & Attached to a white board for display. \\
\hline
\end{tabular}

Ref. Sasaki, 1929: 29, text figs. 9, 10, Pl. VIII figs. 17-19.

\section{Incirrata: Octopodidae}

Amphioctopus cf. aegina (Gray, 1849).

(Genus Amphioctopus Fischer, 1832, revived by Gleadall, 2002, 2003a, $b$; redescription submitted).

\begin{tabular}{llll}
\hline Reg. No. & \multicolumn{1}{c}{ Specimen(s) } & \multicolumn{1}{c}{ Collection data } & \multicolumn{1}{c}{ Comments } \\
\hline $\mathbf{9 9 6 . 9 . 2}$ & 8 males $(37-45)$, & Loc. Tokyo, Tsukiji market.* & All specimens have a thin chevron head bar. \\
{$[\mathbf{1 0 2}]$} & 4 females (40-49). & Coll. 17.ix.1996, I.G.Gleadall. & Pseudophallus type C (Gleadall, 2002). \\
\hline
\end{tabular}

*Probably not from Japanese waters. Condition fair, despite prompt fixation: probably deep-frozen and thawed (imported from S.E. Asia?).

Amphioctopus fangsiao (d'Orbigny, 1841).

\begin{tabular}{|c|c|c|c|}
\hline Reg. No. & Specimen(s) & Collection data & Comments \\
\hline $\begin{array}{l}\text { 994.CO-03 } \\
{[275]}\end{array}$ & 1 male $(41)$ & Loc. [Kyushu], Nagasaki. & $\begin{array}{l}\text { Syntype of Octopus fangsiao fangsiao (origi- } \\
\text { nally as Polypus fangsiao typicus Sasaki, 1929: } \\
\text { 53). Illustrated Pl. III figs. 19, } 20 \text { of Sasaki, } \\
\text { 1929.* }\end{array}$ \\
\hline $\begin{array}{l}\text { 994.CO-04 } \\
{[270]}\end{array}$ & $\begin{array}{l}4 \text { males }(49,38,36, \\
31), 6 \text { females }(50, \\
48,44,43,42,38)\end{array}$ & $\begin{array}{l}\text { Loc. Tokyo Bay, Ohmori [nr. Haneda]. } \\
\text { Coll. 7.v.1906, Kitaro Shimizu. }\end{array}$ & Syntypes of Octopus fangsiao fangsiao. \\
\hline $\begin{array}{l}\text { 994.CO-05 } \\
{[271]}\end{array}$ & $\begin{array}{l}4 \text { females }(51,50,48, \\
22): 3 \text { mature with } \\
\text { vitellogenic eggs, } \\
1 \text { immature }\end{array}$ & $\begin{array}{l}\text { Loc. Tokyo market. } \\
\text { Coll. 7.xii.1882 (M 15). }\end{array}$ & $\begin{array}{l}\text { Syntypes of } O \text {. fangsiao fangsiao. Octopus } \\
\text { ocellatus lot \#1 of Ikeda (1891a: } 25 \text {; as } 2 \\
\text { males, } 2 \text { females). }\end{array}$ \\
\hline $\begin{array}{l}\text { 994.CO-06 } \\
{[272]}\end{array}$ & $\begin{array}{l}1 \text { female ( } 38 \text {, mature } \\
\text { with eggs). }\end{array}$ & $\begin{array}{l}\text { Loc. [Kyushu], Bungo [Oita Pref.], Beppu. } \\
\text { Coll. March, 13.iii.1899 (M 32), Terasaki. }\end{array}$ & $\begin{array}{l}\text { Syntype of } O \text {. fangsiao fangsiao. Originally } \\
\text { with } O \text {. minor } 994 . \mathrm{CO}-07 \text {. }\end{array}$ \\
\hline $\begin{array}{l}996.3 .1 \\
{[041]}\end{array}$ & 2 males $(38,36)$ & $\begin{array}{l}\text { Loc. [Kyushu, Ariake Sea, Fukuoka Pref., } \\
\text { Yanagawa City], Okinohata fish market. } \\
\text { Coll. 8.x.1902? }\end{array}$ & $\begin{array}{l}\text { ?Syntypes of } O \text {. fangsiao fangsiao ( } c f \text {. Sasaki's } \\
\text { lot 'Fukuoka'). }\end{array}$ \\
\hline $\begin{array}{l}\text { 994.CO-08 } \\
{[034]}\end{array}$ & 1 ?female (18) & Loc. not stated. Coll. 1879 (M 12). & $\begin{array}{l}\text { '1 small specimen } O \text {. ocellatus.' Poor condition. } \\
\text { With two specimens of 'Octopus' minor. }\end{array}$ \\
\hline $\begin{array}{l}996.3 .2 \\
{[042]}\end{array}$ & $\begin{array}{l}1 \text { female } \\
(49, \text { mature })\end{array}$ & 'Oshima Research Laboratory, 1950’. & Recent material. \\
\hline $\begin{array}{l}996.3 .3 \\
{[043]}\end{array}$ & 1 male (37). & $\begin{array}{l}\text { Coll. 1-5.iv.1960, East China Sea Agriculture, } \\
\text { Forestry and Fisheries. Loc. Area 319-320. }\end{array}$ & $\begin{array}{l}\text { Recent material. ('Octopus areolatus, } \\
\text { yotsumedako'). }\end{array}$ \\
\hline $\begin{array}{l}\text { 996.3.6.1 } \\
{[047]}\end{array}$ & $\begin{array}{l}3 \text { males }(51,37,35) \text {, } \\
1 \text { female }(29) \text {. }\end{array}$ & (no data) & Recent material? \\
\hline $\begin{array}{l}996.3 .7 \\
{[050]}\end{array}$ & 1 male (20.5), juv. & (no data) & $\begin{array}{l}\text { Very small but each of arms } 2 \text { and } 3 \text { has two } \\
\text { massive suckers. }\end{array}$ \\
\hline $\begin{array}{l}996.3 .13 .2 \\
{[280-2]}\end{array}$ & 1 ? female $(21)$ & $\begin{array}{l}\text { Loc. Kanagawa. Coll. 12.vi.1906 } \\
\text { (M 39) }\end{array}$ & $\begin{array}{l}\text { Sa } 39 \text {. Poor condition. Originally with } 6 \mathrm{Eu} \text { - } \\
\text { prymna berryi. }\end{array}$ \\
\hline $\begin{array}{l}997.6 .1 \\
{[175]}\end{array}$ & 1 female $(46)$ & Loc. [Hokkaido, Hakodate?]. & $\begin{array}{l}\text { Poor condition. (Recent material?). Originally } \\
\text { with Octopus sp. 996.5.42. }\end{array}$ \\
\hline $\begin{array}{l}000.3 .31 .1 \\
{[219]}\end{array}$ & 1 female (c. 55). & (no data) & $\begin{array}{l}\text { A lot including also one 'Octopus' longispadi- } \\
\text { ceus and two 'Octopus' minor. }\end{array}$ \\
\hline
\end{tabular}


Amphioctopus fangsiao, continued

\begin{tabular}{llll}
\hline \multicolumn{1}{c}{ Reg. No. } & \multicolumn{1}{c}{ Specimen(s) } & \multicolumn{1}{c}{ Collection data } \\
\hline $\mathbf{0 0 0 . 3 . 3 1 . 4 . 1}$ & 1 male & Loc. [Ishikawa Pref.], Togi. & (not part of Sasaki's collection). Originally with \\
{$[\mathbf{2 2 2}-1]$} & (38, submature). & Coll. 24.9.1927, Uchida. & a Loliolus japonicus. \\
$\mathbf{0 0 2 . 1 2 . 4 . 4}$ & 1 female & (no data) & ('Octopus sp.').
\end{tabular}

(37, submature)

Sent by postal freight to I. Gleadall by Y. Yamazaki of Tsukiji market, Tokyo. Delivered to Tokyo by Yamaharu Co., Urayasu, Chiba 12 males (34-58, mature)

Pref., in boxes labelled 'Fukushima Pref.,

Iwaki.' (imported?).

Ref. Sasaki, 1929: 49, text figs. 18, 19, Pl. X figs. 1a, b, 2a-c, 3a,b. *Polypus is a suppressed name (ICZN, 1954). The legend to Pl. III figs. 19 and 20 describes this specimen as subspecies Polypus fangsiao etchuanus but Sasaki qualified this identification on p. 55 as 'with great doubt', so this specimen is excluded as type material for variety [subspecies] etchuanus. Sasaki included the locality 'Nagasaki' among specimens inspected by him for both subspecies but this is the only known specimen of Sasaki's from Nagasaki. It therefore appears that Sasaki included it among the specimens by which he defined $O$. fangsiao fangsiao (as P. f. typicus).

Amphioctopus cf. kagoshimensis* (Ortmann, 1888) (Octopus granulatus Lamarck sensu Sasaki, 1929).

\begin{tabular}{|c|c|c|c|}
\hline Reg. No. & Specimen(s) & Collection data & Comments \\
\hline $\begin{array}{l}\text { 994.CO-23.1 } \\
{[099-1]}\end{array}$ & $\begin{array}{l}1 \text { male }(84) \\
1 \text { female }(102)\end{array}$ & (no data) & $\begin{array}{l}\text { Female is distorted and reproductive system has } \\
\text { been removed. Originally with two } O \text {. vulgaris. }\end{array}$ \\
\hline $\begin{array}{l}\text { 994.CO-32.2 } \\
{[023-2]}\end{array}$ & 1 male ( 40, mature) & $\begin{array}{l}\text { Loc. [Kyushu] Kagoshima fish } \\
\text { market. Coll. 29.iii.1896, } \\
\text { Mitsukuri and Hara, via Mr. Miyajima. }\end{array}$ & $\begin{array}{l}\text { cf. Sasaki, 1929: } 41 \text { 'with great hesitation.' } \\
\text { Illustrated by Sasaki, 1929: Pl. III fig. 18. Male } \\
\text { reproductive system illustrated in Pl. IX fig. } 13 . \\
\text { ('Octopus vulgaris'). } \\
\text { Included with one Octopus cf. vulgaris. }\end{array}$ \\
\hline $\begin{array}{l}\text { 994.CO-36 } \\
{[027]}\end{array}$ & $\begin{array}{l}3 \text { males }(69,59,53, \\
\text { mature }) ; 2 \text { females } \\
(52,42, \text { submature })\end{array}$ & $\begin{array}{l}\text { Loc. Kagoshima. Coll. 29.iii. } 1896 \\
\text { (M 29), Mitsukuri and Hara. }\end{array}$ & $\begin{array}{l}\text { 'Polypus granulatus Lam.' Loc. listed by Sasaki, } \\
\text { 1929: } 40 .\end{array}$ \\
\hline $\begin{array}{l}\text { 994.CO-37 } \\
{[028]}\end{array}$ & $\begin{array}{l}5 \text { males }(83,72,70, \\
67,63) .\end{array}$ & $\begin{array}{l}\text { Loc. Boshu [Chiba Pref.]. Coll. May, 1906, } \\
\text { bought from Kintaro Shimizu. }\end{array}$ & (ditto) \\
\hline $\begin{array}{l}001.10 .2 .2 \\
{[053]}\end{array}$ & 1 male (45) & Coll. $1960 ?$ & $\begin{array}{l}\text { Very small ligula }(4 \mathrm{~mm}) \text {. Originally with one } \\
\text { M. loennbergii, two chiroteuthids and a loligi- } \\
\text { nid. "' } 60-355 \text { " [coll. 1960?; Lot \#355?]. }\end{array}$ \\
\hline
\end{tabular}

Ref. Sasaki, 1929: 40, Pl. III fig. 18, Pl. IX figs. 11-13, Pl. XXIX figs. 2, 3. See also present paper under Octopus cf. vulgaris. *Some recent labels use the spelling 'kagoshimaensis' but the final 'a' of Kagoshima should not be included (cf. China and species group name chinensis).

Amphioctopus marginatus (Taki, 1964).

\begin{tabular}{llcc}
\hline Reg. No. & Specimen(s) & Collection data & Comments \\
\hline 994.CO-39 & 1 male $(81)$ & 1 old field label: ' $34^{\circ} 9^{\prime} 23^{12}$ [Area] 330. tako.' & Arms IV truncated. Colour very dark. \\
[100] & & & \\
\hline
\end{tabular}

cf. Taki, 1964: 304, text figs. 47-48, Pl. 5 figs. 1, 2.

\begin{tabular}{|c|c|c|c|}
\hline Reg. No. & Specimen(s) & Collection data & Comments \\
\hline $\begin{array}{l}\text { 994.CO-30 } \\
{[021]}\end{array}$ & $\begin{array}{l}1 \text { male }(21) \\
1 \text { juv. }(7.5)\end{array}$ & $\begin{array}{l}\text { Loc. Chôsen Kaikyô [Straits of Korea]. Coll. Mr. } \\
\text { Kumada (undated). }\end{array}$ & - \\
\hline $\begin{array}{l}996.3 .6 .2 \\
{[048]}\end{array}$ & 1 female (46) & (no data) & (recent material?) \\
\hline
\end{tabular}

cf. Sasaki, 1917b: 364; 1929: 55, text figs. 20, 21, Pl. X figs. 11-15 (as Polypus ovulum).

\begin{tabular}{lcll}
\multicolumn{2}{l}{ Amphioctopus robsoni (Adam, 1941). } \\
\hline Reg. No. & Specimen(s) & \multicolumn{1}{c}{ Collection data } & Comments \\
\hline $\mathbf{9 9 6 . 9 . 1}$ & 15 males (36-45), & Loc. Tokyo, Tsukiji market. & Probably not from Japanese waters. Condition \\
{$[101]$} & 2 females (44, 42, & Coll. 17.ix.1996, I.G.Gleadall. & $\begin{array}{l}\text { fair, despite prompt fixation: probably deep- } \\
\text { frozen and thawed (imported from S.E. Asia?). }\end{array}$
\end{tabular}

These were purchased in an attempt to obtain more specimens of A. ovulum, since 'Tokyo market' was the source of the type specimens listed by Sasaki $(1917 b, 1929)$. Although recorded in the scientific literature as a species apparently confined to the Red Sea, A. robsoni is in fact abundant throughout the tropical Indo-West Pacific region (Gleadall, 2003b.; redescription submitted.; cf. Gleadall, 2002: 72). 
Amphioctopus sp. (without ocelli).

\begin{tabular}{|c|c|c|c|}
\hline Reg. No. & Specimen(s) & Collection data & Comments \\
\hline $\begin{array}{l}\text { 994.CO-22 } \\
{[014]}\end{array}$ & $\begin{array}{l}1 \text { female }(30) \text {, } \\
2 \text { juv. }(17,9) \text {. }\end{array}$ & $\begin{array}{l}\text { Loc. [Seto Sea], Bingo [Hiroshima } \\
\text { Pref.] Tomonotsu. Coll. summer, } \\
1882 \text { [Mitsukuri, fide Ikeda]. }\end{array}$ & $\begin{array}{l}\text { cf. Octopus octopodia lot. \#2 of Ikeda, 1890a: } 481 . \\
\text { ('Octopus kagoshimensis'). }\end{array}$ \\
\hline $\begin{array}{l}\text { 994.CO-35 } \\
{[026]}\end{array}$ & 1 female (57) & Loc. [Kyushu], Kagoshima market. & Originally with three Octopus cf. vulgaris. \\
\hline
\end{tabular}

Hapalochlaena fasciata (Hoyle, 1886).

\begin{tabular}{|c|c|c|c|}
\hline Reg. No. & Specimen(s) & Collection data & Comments \\
\hline $\begin{array}{l}\text { 994.CO-17 } \\
{[273]}\end{array}$ & 1 female $(37)$ & $\begin{array}{l}\text { Loc. Aburatsubo Bay, Misaki. } \\
\text { Coll. 4.i.1898, Kumakichi. }\end{array}$ & Illustrated in Sasaki (1929) Pl. XI fig. 1. \\
\hline $\begin{array}{l}\text { 994.CO-18 } \\
{[274]}\end{array}$ & 2 females $(20,8)$. & $\begin{array}{l}\text { Loc. Kanagawa Pref. Misaki. } \\
\text { Coll. Aug. 1897. (M.30) }\end{array}$ & $\begin{array}{l}\text { Sasaki, 1929: 58; and cf. } 1 \text { male (VML 26), } 1 \text { juv. (13), } \\
\text { fide Sasaki, 1929: 60. Found dried out (VML 16, 7). }\end{array}$ \\
\hline
\end{tabular}

Inspection of the holotype of $H$. fasciata provides no reason to doubt that these Japanese specimens are the same species, despite the punctuated distribution of known specimens in Honshu (Japan), Hong Kong and eastern Australia (cf. Norman and Hochberg, 1994; Gleadall, 1997). Ref. Sasaki, 1929: 58, Pl. XI figs. 1-3 (as Polypus fasciatus). cf. Hoyle, 1886: 94, Pl. VIII fig. 3; Roper and Hochberg, 1988: 167, figs. 11, 12, Table 5.

'Octopus' ${ }^{\text {' }}$ minor (Sasaki, 1920) (Subspecies distinctions not supported). (O. macropus group)

\begin{tabular}{|c|c|c|c|}
\hline Reg. No. & Specimen(s) & Collection data & Comments \\
\hline $\begin{array}{l}\text { 994.CO-07 } \\
{[035]}\end{array}$ & 1 female $(65)$ & $\begin{array}{l}\text { Loc. [Kyushu], Bungo [Oita Pref.], } \\
\text { Beppu. Coll. 13.iii.1899 (M 32), } \\
\text { Terasaki. }\end{array}$ & $\begin{array}{l}\text { Syntype of Polypus variabilis typicus Sasaki, } 1929 \\
\text { (= Octopus minor variabilis). 'Octopus macropus Risso.' } \\
\text { Originally with A. fangsiao 994.CO-06. }\end{array}$ \\
\hline $\begin{array}{l}\text { 994.CO-09 } \\
{[237]}\end{array}$ & $\begin{array}{l}1 \text { male }(45) \text {, } \\
1 \text { female (49). } \\
\text { (both immature). }\end{array}$ & $\begin{array}{l}\text { Loc. [Seto Sea] Bitchu } \\
\text { [Okayama Pref.], } \\
\text { Yukashima. Coll. summer, } 1882 \\
\text { (M 15), Kikuchi. }\end{array}$ & $\begin{array}{l}\text { Syntypes of } O \text {. minor variabilis (see above) 'Octopus } \\
\text { cuvier Orbigny.' Ikeda, } 1890 \mathrm{~b}: 529, \text { O. macropus lot \#5. }\end{array}$ \\
\hline $\begin{array}{l}\text { 994.CO-10 } \\
{[036]}\end{array}$ & 6 males $(61-72)$ & $\begin{array}{l}\text { Loc. Tokyo Bay, Haneda. } \\
\text { Coll. 21.vi.1882. }\end{array}$ & $\begin{array}{l}\text { Syntypes of } O . \text { m. variabilis (see above). } c f . \text { O. macropus } \\
\text { lot \#1 of Ikeda, 1890b: } 529 \text {. }\end{array}$ \\
\hline $\begin{array}{l}\text { 994.CO-11 } \\
{[037]}\end{array}$ & $\begin{array}{l}1 \text { male }(72, \text { mature }) \text {, } \\
2 \text { females }(60,56) .\end{array}$ & $\begin{array}{l}\text { Loc. Tokyo market. } \\
\text { Coll. 7.xii.1882. }\end{array}$ & $\begin{array}{l}\text { Syntypes of } O . \text { m. variabilis (see above). 'O. pusillus } \\
\text { Gould.' 'Polypus macropus Risso.' O. macropus lot \#2 of } \\
\text { Ikeda, 1890b: } 528-529 \text {. }\end{array}$ \\
\hline $\begin{array}{l}\text { 994.CO-16 } \\
{[045]}\end{array}$ & $\begin{array}{l}2 \text { females } \\
(63,50, \text { submature }) .\end{array}$ & $\begin{array}{l}\text { Loc. [Seto Sea, Okayama } \\
\text { Pref.], Kojima Bay. } \\
\text { Coll. 26.xii.1906. }\end{array}$ & $\begin{array}{l}\text { Syntypes of } O . \text { m. variabilis (see above). 'Polypus } \\
\text { macropus Risso.' Small ovary and small, developing } \\
\text { eggs. }\end{array}$ \\
\hline $\begin{array}{l}996.3 .9 \\
{[055]}\end{array}$ & $\begin{array}{l}2 \text { males }(84,76), \\
1 \text { female }(96)\end{array}$ & $\begin{array}{l}\text { Loc. Tokyo Bay. } \\
\text { Coll. 26.iv.1906. }\end{array}$ & Syntypes of $O . m$. variabilis (see above). \\
\hline $\begin{array}{l}\text { 994.CO-08 } \\
{[034]}\end{array}$ & 2 ?females $(31,26)$ & $\begin{array}{l}\text { Loc. not stated. } \\
\text { Coll. } 1879 \text { (M 12). }\end{array}$ & $\begin{array}{l}\text { 'O. pusillus' (cf. Ikeda, 1890b: 528). With one specimen } \\
\text { of A. fangsiao. }\end{array}$ \\
\hline $\begin{array}{l}\text { 994.CO-15 } \\
{[040]}\end{array}$ & 4 males $(85,77,75,69)$. & Loc. Haneda. Coll. 25.iv.1906. & $\begin{array}{l}\text { Dissected. }{ }^{2} \text { 'Polypus macropus.' 'Octopus macropus } \\
\text { Risso.' }\end{array}$ \\
\hline $\begin{array}{l}\text { 994.CO-45 } \\
{[003]}\end{array}$ & 1 male ( 85, mature). & (no data) & - \\
\hline $\begin{array}{l}997.7 .1 \\
{[176]}\end{array}$ & $\begin{array}{l}1 \text { female } \\
\text { (83, mature with eggs). }\end{array}$ & (no data) & $\begin{array}{l}\text { Eggs are vitellogenic (length approx. } 11 \mathrm{~mm} \text { ). Label ' } 24 \text { ' } \\
\text { tied to specimen. }\end{array}$ \\
\hline $\begin{array}{l}000.3 .31 .1 \\
{[219]}\end{array}$ & 2 ?females $(80,56)$ & (no data) & $\begin{array}{l}\text { Poor condition. With one A. fangsiao and one 'Octopus' } \\
\text { longispadiceus. }\end{array}$ \\
\hline $\begin{array}{l}000.4 .28 .3 .1 \\
{[223-1]}\end{array}$ & 2 males $(69,68)$. & (no data) & $\begin{array}{l}\text { From a mixed lot with two species of Sepia, Sepiella } \\
\text { japonica and Loliolus japonicus. }\end{array}$ \\
\hline
\end{tabular}

Ref. Sasaki, 1929: 90, text figs. 47, 48, Pl. I fig. 9, Pl. III fig. 21, Pl. XII figs. 13, 14a, b. ${ }^{1}$ Eledonenta filholiana Rochebrune, 1884b, was identified by Norman, 1993: 306, as a member of the 'Octopus' macropus group, based upon which Eledonenta is a candidate genus name for this group of octopuses. However, subsequent papers by Norman have not used this identification (e.g., Norman and Sweeney, 1997) and no alternative name has been published. ${ }^{2}$ Reproductive organs removed from two specimens. Only one has an intact ligula (others still have distinct copulatory groove down arm 3 ). 
'Octopus' oshimai (Sasaki, 1929). (O. macropus group)

\begin{tabular}{|c|c|c|c|}
\hline Reg. No. & Specimen(s) & Collection data & Comments \\
\hline $\begin{array}{l}\text { 994.CO-13 } \\
{[038]}\end{array}$ & $\begin{array}{l}1 \text { female } \\
(50, \text { mature with eggs) }\end{array}$ & $\begin{array}{l}\text { Loc. [Taiwan Straits], Hôkôto (Hôkô Islands, } \\
=\text { P'eng-hu Lieh-tao, = Pescadores). } \\
\text { Coll. } 1897 \text { ? }\end{array}$ & $\begin{array}{l}\text { Poor condition. 'Polypus macropus Risso?' } \\
\text { ('Octopus minor'). }\end{array}$ \\
\hline
\end{tabular}

Ref. Sasaki, 1929: 44, text fig. 16, Pl. XXVIII figs. 1-5, Pl. XXX fig. 12.

‘Octopus’ pardalis (Sasaki, 1929). (O. macropus group)

\begin{tabular}{llll}
\hline Reg. No. & \multicolumn{1}{c}{ Specimen(s) } & \multicolumn{1}{c}{ Collection data } & Comments \\
\hline 994.CO-19 & 1 male & Loc. Awa Province [Chiba Pref.], Kominato. & Holotype by monotypy. Ligula missing. \\
{$[\mathbf{2 3 8}]$} & $(51$, submature). & Coll. Apr., 1885 (M.18), Ishikawa. & O. macropus lot \#4 of Ikeda, 1890b: 529. \\
\hline
\end{tabular}

Ref. Sasaki, 1929: 94; Pl. XII figs. 15, 16 (as Polypus variabilis pardalis).

'Octopus' sp. (O. macropus group)

\begin{tabular}{ccll}
\hline Reg. No. & \multicolumn{1}{c}{ Specimen(s) } & \multicolumn{1}{c}{ Collection data } & \multicolumn{1}{c}{ Comments } \\
\hline $\begin{array}{l}\text { 994.CO-14 } \\
{[\mathbf{0 3 9}]}\end{array}$ & 1 male $(121)$. & Loc. Misaki, 130 hiro $(\mathrm{fm})$ & $c f$. O. minor but ligula is different (regenerating?) \\
& & {$[238 \mathrm{~m}]$. Coll. Kuma. } & and no papillae above eyes. \\
\hline
\end{tabular}

'Octopus' longispadiceus (Sasaki, 1917) (not genus Octopus Cuvier or the 'Octopus macropus group')*.

\begin{tabular}{cccc}
\hline Reg. No. & Specimen(s) & Collection data & \multicolumn{1}{c}{ Comments } \\
\hline $\mathbf{0 0 0 . 3 . 3 1 . 1}$ & 1 male $(50)$. & (no data) & Poor condition. With a female A. fangsiao and two \\
{$[219]$} & & & 'Octopus' minor. \\
\hline
\end{tabular}

Ref. Sasaki, 1917b: 366; 1929: 79, text fig. 38, Pl. XI figs. 17-19; Gleadall, 1993: 153. *In size, gross morphology and in the anatomy of the pseudophallus, this species most closely resembles the inkless Atlantic species Muusoctopus januarii (Hoyle): see Gleadall (2004).

Octopus parvus (Sasaki, 1917).

\begin{tabular}{lccc}
\hline Reg. No. & Specimen(s) & Collection data & \multicolumn{1}{c}{ Comments } \\
\hline $\mathbf{9 9 6 . 3 . 5}$ & 1 female (18, mature). & (no data) & '- teaching lab. specimen' (in Japanese). Labels \\
[046] & & illegible. '5' ('Octopus berenice').* &
\end{tabular}

Ref. Sasaki, 1917b: 365; 1929: 38 (as Polypus parvus). *Different from O. berenice Gray, 1849.

Octopus cf. vulgaris Cuvier, 1797 (cf. O. sinensis d'Orbigny, 1841).

\begin{tabular}{|c|c|c|c|}
\hline Reg. No. & Specimen(s) & Collection data & Comments \\
\hline $\begin{array}{l}\text { 994.CO-23.2 } \\
{[099-2]}\end{array}$ & $\begin{array}{l}1 \text { male }(48) \\
1 \text { female }(63)\end{array}$ & (no data) & $\begin{array}{l}\text { Originally with two A. cf. } \\
\text { kagoshimensis (see 994.CO-23.1). }\end{array}$ \\
\hline $\begin{array}{l}\text { 994.CO-24 } \\
{[015]}\end{array}$ & 1 ?female (35). & $\begin{array}{l}\text { Loc. Echizen [Fukui Pref.], Sakai Port. } \\
\text { Coll. July, } 1885 \text { (M 18), [Okada, fide Ikeda]. }\end{array}$ & $\begin{array}{l}\text { This locality (and 'Ikeda') listed by Sasaki, } \\
\text { 1929. Ikeda, 1890a: } 479 \text {, Octopus octopodia lot } \\
\text { \#6. Beaks removed. }\end{array}$ \\
\hline $\begin{array}{l}\text { 994.CO-26 } \\
{[017]}\end{array}$ & 1 female (52). & $\begin{array}{l}\text { Loc. Boshu [Chiba Pref.]. Coll. 7.v.1906 M 39), } \\
\text { Kentaro Shimizu. }\end{array}$ & Body twisted to right side. \\
\hline $\begin{array}{l}\text { 994.CO-27 } \\
{[018]}\end{array}$ & 1 male ( 90, mature). & Loc. [Kyushu], Kagoshima. (undated). & $\begin{array}{l}\text { 'Octopus granulatus Lam.' cf. Sasaki, 1929: } 41 \text {, } \\
\text { Pl. IX fig. } 2 \text {. Dissected male organs included in a } \\
\text { separate container. }\end{array}$ \\
\hline $\begin{array}{l}\text { 994.CO-28 } \\
{[019]}\end{array}$ & 1 male (49). & $\begin{array}{l}\text { Loc. [Japan Sea], Tango Kuni [Kyoto Pref.], } \\
\text { Miyazu Bay. Coll. 6.vii.1903 (M 36), Iizuka. }\end{array}$ & Sa 66. This locality listed by Sasaki, 1929. \\
\hline $\begin{array}{l}\text { 994.CO-29 } \\
{[020]}\end{array}$ & 1 female $(70)$ & $\begin{array}{l}\text { Loc. [Kanagawa Pref.], nr. Misaki Marine } \\
\text { Biological Station. } \\
\text { Coll. Mid-Aug., } 1902 \text { (M 35). }\end{array}$ & $\begin{array}{l}\text { 'octopodia?' 'Purchased from near the Misaki } \\
\text { Experimental [Marine Biological] Station.' } \\
\text { Good condition. Arm L1 regenerating at base. }\end{array}$ \\
\hline $\begin{array}{l}\text { 994.CO-32.1 } \\
{[023-1]}\end{array}$ & 1 male ( 37, submature) & $\begin{array}{l}\text { Loc. [Kyushu] Kagoshima fish } \\
\text { market. Coll. 29.iii.1896, Mitsukuri and Hara, } \\
\text { via Mr. Miyajima. }\end{array}$ & $\begin{array}{l}\text { Originally with one } A \text {. cf. } \\
\text { kagoshimensis. }\end{array}$ \\
\hline $\begin{array}{l}\text { 994.CO-35 } \\
{[026]}\end{array}$ & $\begin{array}{l}1 \text { male }(47), 2 \text { females } \\
(51,49) . \text { Submature. }\end{array}$ & Loc. [Kyushu], Kagoshima market. & $\begin{array}{l}\text { Opened and partly dissected. With one } \\
\text { Amphioctopus sp. ('Octopus madokai } \\
\text { sagamidako'). }\end{array}$ \\
\hline $\begin{array}{l}\text { 994.CO-38 } \\
{[056]}\end{array}$ & $\begin{array}{l}1 \text { male } \\
(107, \text { submature })\end{array}$ & $\begin{array}{l}\text { Loc. [Kyushu], Kagoshima } \\
\text { Coll. Aug., } 1903 \text { (M 36), Kagoshima Pref. Govt. } \\
\text { (donation). }\end{array}$ & $\begin{array}{l}\text { This locality listed by Sasaki, } 1929 . \text { 'Polypus } \\
\text { vulgaris Lam., Kagoshima.' 'Octopus granula- } \\
\text { tus Lam., Kagoshima.' }\end{array}$ \\
\hline
\end{tabular}


Octopus cf. vulgaris, continued

\begin{tabular}{|c|c|c|c|}
\hline Reg. No. & Specimen(s) & Collection data & Comments \\
\hline $\begin{array}{l}996.3 .4 \\
{[044]}\end{array}$ & 1 male $(59$, mature $)$. & (no clear data) & '51-52 天 9-13 入港' \\
\hline $\begin{array}{l}996.3 .6 .3 \\
{[049]}\end{array}$ & 1 female (63). & (no data) & Poorly fixed. (not Sasaki material?). \\
\hline $\begin{array}{l}\text { 001.10.1 } \\
{[031]}\end{array}$ & 1 juv. (9). & $\begin{array}{l}\text { Loc. Kagoshima Bay, Osumi, Sakurajima, in } \\
\text { front of Kurokami. Coll. 1.iv.1896 (M 29), } \\
\text { Mitsukuri and Hara. }\end{array}$ & $\begin{array}{l}\text { This locality listed by Sasaki, 1929, both as } \\
\text { 'Sakurajima' and 'Osumi.' }\end{array}$ \\
\hline $\begin{array}{l}001.10 .5 \\
{[030]}\end{array}$ & 1 juv. (29). & (no data) & 'Octopus vulgaris Lam.' \\
\hline
\end{tabular}

Ref. Sasaki, 1929: 35, text figs. 11, 12, Pl. IV fig. 1, Pl. IX figs. 1-3, Pl. XXIX fig. 1.

Unidentified Octopodidae

\begin{tabular}{|c|c|c|c|}
\hline Reg. No. & Specimen(s) & Collection data & Comments \\
\hline $\begin{array}{l}\text { 994.CO-12 } \\
{[001]}\end{array}$ & 2 males $(26,20)$. & $\begin{array}{l}\text { Loc. [Taiwan Straits], Hôkôto [Hôkô Islands, } \\
=\text { P'eng-hu Lieh-tao, = Pescadores]. } \\
\text { Coll. Jan. } 1897 \text { (M 30). }\end{array}$ & $\begin{array}{l}\text { 'Polypus macropus.' Small, globular mantle; head } \\
\text { almost as broad as mantle. ('Taiwan dako. ?Octopus } \\
\text { oshimai'). }\end{array}$ \\
\hline $\begin{array}{l}\text { 994.CO-20 } \\
{[058]}\end{array}$ & $\begin{array}{l}1 \text { female }(119) \text {, } \\
1 \text { male }(106, \\
\text { mantle only) }\end{array}$ & (no data) & $\begin{array}{l}\text { O. ? hongkongensis Hoyle sensu Gleadall, 1993: 148, } \\
\text { Table } 1 \text {, ref. \#92/2. }\end{array}$ \\
\hline $\begin{array}{l}\text { 994.CO-21 } \\
{[054]}\end{array}$ & 1 female (124) & $\begin{array}{l}\text { Loc. [Kyushu], Kagoshima. } \\
\text { Coll. Aug., } 1903 \text { (M 36). }\end{array}$ & $\begin{array}{l}\text { Gift from Kagoshima Pref. Govt. O. ? hongkongensis } \\
\text { Hoyle sensu Gleadall, 1993: 148, Table 1, ref. } \\
\# 92 / 1 \text {. }\end{array}$ \\
\hline $\begin{array}{l}\text { 994.CO-25 } \\
{[016]}\end{array}$ & 1 juv. (19). & (no data) & Several arms truncated. 'O. cuvieri young.' \\
\hline $\begin{array}{l}\text { 994.CO-31 } \\
{[022]}\end{array}$ & $\begin{array}{l}1 \text { ?male (29) } \\
1 \text { juv. (12). }\end{array}$ & $\begin{array}{l}\text { Loc. Boshu [Chiba Pref.], Kominato Coll. Apr., } \\
1885 \text { (M.18), [Ishikawa, Okada, fide Ikeda]. }\end{array}$ & $\begin{array}{l}\text { This locality listed by Sasaki, 1929, for O. vulgaris. } \\
\text { cf. Octopus octopodia lot \#8 of Ikeda, 1890a: } 481 .\end{array}$ \\
\hline $\begin{array}{l}\text { 994.CO-33 } \\
{[024]}\end{array}$ & $\begin{array}{l}1 \text { male }(29) \text {, } \\
1 \text { juv. (19). }\end{array}$ & $\begin{array}{l}\text { Loc. Suruga [Shizuoka Pref., Numazu City], } \\
\text { Enoura. Coll. Apr. } 1884 \text { (M 17). }\end{array}$ & $\begin{array}{l}\text { This locality listed by Sasaki, 1929, for O. vulgaris. } \\
\text { Octopus octopodia lot \#3 of Ikeda, 1890a: 481. cf. } \\
\text { 994.CO-12. }\end{array}$ \\
\hline $\begin{array}{l}\text { 994.CO-34 } \\
{[025]}\end{array}$ & $\begin{array}{l}1 \text { female } \\
(22, \text { submature }) \text {. }\end{array}$ & $\begin{array}{l}\text { Loc. [Kyushu], Hyuga [Miyazaki Pref.], Ariake } \\
\text { Bay, Natsu Beach. Coll. 13.iv.1896, Mitsukuri } \\
\text { and Hara. }\end{array}$ & $\begin{array}{l}\text { Jar labels include 'octopodia' [cf. Octopus vulgaris] } \\
\text { and 'Octopus granulatus'. ('Octopus kagoshimensis. } \\
\text { Sunadako'). }\end{array}$ \\
\hline $\begin{array}{l}996.3 .8 \\
{[051]}\end{array}$ & $\begin{array}{l}1 \text { female }(30) \\
\text { with small eggs. }\end{array}$ & Loc. not given 'Saghalien Collection, 1906.' & $\begin{array}{l}\text { Arms subequal. ('Octopus berenice Gray, } \\
\text { mamedako'). }\end{array}$ \\
\hline $\begin{array}{l}996.5 .42 \\
{[029]}\end{array}$ & 1 male (46). & Loc. [Hokkaido, Hakodate?]. & $\begin{array}{l}\text { 'Holotype' of sp. } 1 \text {. (unpublished). }{ }^{1} \text { Wooden tag ' } 42 \text { ' } \\
\text { (in Chinese characters). Originally with } 1 \text { female } \\
\text { A. fangsiao (997.6.1). ('Octopus hokkaidensis'). }{ }^{2}\end{array}$ \\
\hline $\begin{array}{l}996.5 .43 \\
{[078]}\end{array}$ & $\begin{array}{l}2 \text { females (both } 38 \text {, } \\
\text { immature). }\end{array}$ & $\begin{array}{l}\text { Loc. [Kyushu], Kagoshima market. } \\
\text { Coll. 29.iii.1896, Mitsukuri and Hara }\end{array}$ & 'Co-type' of sp. 2 (unpublished). ${ }^{1}$ \\
\hline $\begin{array}{l}996.5 .44 \\
{[007]}\end{array}$ & $\begin{array}{l}1 \text { female ( } 40 \text {, } \\
\text { mature with eggs } \\
7.5 \mathrm{~mm} \text { long). }\end{array}$ & Sagami [Kanagawa Pref.], Enoshima. & $\begin{array}{l}\text { 'Co-type' of sp. 2. (unpublished). }{ }^{1} \text { cf. Octopus } \\
\text { macropus lot } \# 6 \text { of Ikeda, 1890b: } 528 \text {. ('probably } \\
\text { O. minor'). }\end{array}$ \\
\hline $\begin{array}{l}000.3 .31 .5 \\
{[227]}\end{array}$ & 1 spec. $(35)$. & 20.vi.1964? & $\begin{array}{l}\text { 'W. } 1500 \mathrm{M} 20 / 6 \text { ' } 39 \text { ' '7'. ('Benthoctopus sp., } \\
\text { chihirodako'). }{ }^{3}\end{array}$ \\
\hline $\begin{array}{l}001.10 .5 \\
{[030]}\end{array}$ & 1 juv. (29). & (no data) & 'Octopus vulgaris Lam.' \\
\hline $\begin{array}{l}002.12 .3 .1 \\
{[006]}\end{array}$ & 1spec. (22) & Loc. Heda. Coll. Dec., 1936. & $\begin{array}{l}\text { ' } 8 \text { '. ('?Octopus megalops'). MW 19; head very large } \\
\text { (HW 20). cf. O. spinosus Sasaki, 1920). }\end{array}$ \\
\hline $\begin{array}{l}003.3 .11 \\
{[005]}\end{array}$ & $\begin{array}{l}1 \text { female ( } 40 \text {, } \\
\text { mature with eggs). }\end{array}$ & $\begin{array}{l}\text { Loc. Izu [Shizuoka Pref.], Kona. } \\
\text { Coll. 14.x.1899, Ôsuton [Owston]. }\end{array}$ & $\begin{array}{l}\text { 'OC-5366.' A gift from Mr. Owston. ('Octopus } \\
\text { luteus (Sasaki, 1929), same-hada tenagadako'). }{ }^{4}\end{array}$ \\
\hline 003.3.12 & $\begin{array}{l}3 \text { juv. } \\
(9.0,7.0,4.5)\end{array}$ & $\begin{array}{l}\text { Loc. Boshu [Chiba Pref.], Kominato. } \\
\text { Coll. April, } 1885 \text { (M 18). }\end{array}$ & Three very small specimens, all different species. \\
\hline $\begin{array}{l}004.8 .1 \\
{[218]}\end{array}$ & 2 males $(21,16)$ & Coll. 14.viii.1906. & Saghalien Collection. 'Chebisani.' '10.' \\
\hline
\end{tabular}

${ }^{1}$ See Introduction. ${ }^{2}$ The type of $O$. hokkaidensis (Berry) $[=$ O. glaber (Sasaki, 1920)] has no ink sac but this specimen has, so identification as 'Octopus hokkaidensis' is doubtful. ${ }^{3}$ Ink sac present. Not a species of Benthoctopus Grimpe, which is a junior synonym of Bathypolypus Grimpe (see Muus, 2002). ${ }^{4}$ This specimen has large eggs, approx. $10 \mathrm{~mm}$ long, so it cannot be 'Octopus' luteus (a macropus group species with small eggs, approx. $1 \mathrm{~mm}$ long). 


\section{Decabrachia: Sepiolida: Sepiolidae}

(Measurements of DML are to the fusion point of the dorsal mantle with the head.)

Euprymna berryi Sasaki, 1929.

\begin{tabular}{|c|c|c|c|}
\hline Reg. No. & Specimen(s) & Collection data & Comments \\
\hline $\begin{array}{l}\text { 996.CD-07 } \\
{[276]}\end{array}$ & 2 females $(33,29)$. & Loc. [Kyushu] Nagasaki. & $\begin{array}{l}\text { Syntypes. Originally with A. fangsiao } \\
994 . \text { CO- } 03 \text {. }\end{array}$ \\
\hline $\begin{array}{l}996.3 .10 \\
{[277]}\end{array}$ & $\begin{array}{l}3 \text { males }(15,13.5,8.0) \text {, } \\
4 \text { females } \\
(22,12,10,9.5) .\end{array}$ & $\begin{array}{l}\text { Loc. [Kyushu], Hyuga Prov. [Miyazaki } \\
\text { Pref.], Hososhima [Bay]. Coll. 15.iii.1899. }\end{array}$ & Syntypes. Sa 47. '12'. \\
\hline $\begin{array}{l}996.3 .11 \\
{[278]}\end{array}$ & 1 female $(25)$ & $\begin{array}{l}\text { Loc. [Japan Sea, Hyogo Pref.], Miyazu Bay. } \\
\text { Coll. 9.vii.1903. }\end{array}$ & Syntype. Sa 42. '13'. \\
\hline $\begin{array}{l}\text { 996.3.12 } \\
{[279]}\end{array}$ & 5 females (31-37). & $\begin{array}{l}\text { Shimo-osa [Chiba Pref.], Horii. } \\
\text { Coll. 2.v.1906. }\end{array}$ & Syntypes. Sa 38. '14'. \\
\hline $\begin{array}{l}\text { 996.3.13.1 } \\
{[280-1]}\end{array}$ & 6 females $(38-50)$. & $\begin{array}{l}\text { Loc. Kanagawa. } \\
\text { Coll. 12.vi.1906 (M 39). }\end{array}$ & $\begin{array}{l}\text { Syntypes. Sa } 39 \text {. ' } 15 \text { '. Originally with one } \\
\text { Amphioctopus fangsiao. }\end{array}$ \\
\hline $\begin{array}{l}002.10 .25 .3 \\
{[252]}\end{array}$ & $\begin{array}{l}1 \text { male }(24) \\
1 \text { female }(25) \text {. }\end{array}$ & $\begin{array}{l}\text { Loc. [Osaka Bay], Awaji [Island], } \\
\text { Tsuna-gun, Sumoto. (undated). }\end{array}$ & $\begin{array}{l}\text { Syntypes. Sa } 45 \text {. 'Euprymna morsei.' cf. } \\
\text { E. morsei lot \#3 of Ikeda, 1891b: 62-63. }\end{array}$ \\
\hline $\begin{array}{l}002.10 .25 .4 \\
{[253]}\end{array}$ & 2 specs. $(21,13)$. & $\begin{array}{l}\text { Loc. Bungo [Oita Pref.], Beppu. } \\
\text { Coll. 13.iii.1899 (M 32), Terasaki. }\end{array}$ & $\begin{array}{l}\text { Syntypes (1 missing). Sa } 48 . \\
\text { 'Euprymna scolopes.' }\end{array}$ \\
\hline $\begin{array}{l}002.10 .25 .5 \\
{[251]}\end{array}$ & 1 male (14). & $\begin{array}{l}\text { Loc. [Kanagawa Pref.], Misaki. } \\
\text { Coll. Dec., } 1882 .\end{array}$ & Syntype. Sa 37. \\
\hline $\begin{array}{l}997.3 .3 \\
{[247]}\end{array}$ & 1 female (21). & $\begin{array}{l}\text { Loc. Sagami Bay, } 6 \text { miles off Misaki. } \\
\text { Coll. 5.viii.1906. }\end{array}$ & $\begin{array}{l}\text { Sa 12. Originally with } 9 \text { specimens of } \\
\text { Eucleoteuthis luminosa. }\end{array}$ \\
\hline
\end{tabular}

Ref. 1929: 143, text fig. 88, Pl. XV figs. 12, 13a, b. See also E. morsei (Verrill) sensu Sasaki, 1913b: 247, text fig. 1; 1914a: 589, Pl. XI figs. 1-4.

Euprymna sp.*

\begin{tabular}{lccl}
\hline \multicolumn{1}{c}{ Reg. No. } & Specimen(s) & Collection data & \multicolumn{1}{c}{ Comments } \\
\hline $\mathbf{0 0 2 . 1 0 . 2 4 . 3}$ & 1 spec. (39) & (no data) & 'Euprymna morsei.' \\
{$\left[\begin{array}{l}\mathbf{2 2 6}] \\
\mathbf{0 0 2 . 1 0 . 2 4 . 4}\end{array}\right.$} & 14 specs. (8-19) & (no data) & 'Euprymna scolopes Berry.' Sa 49. Very \\
{$[\mathbf{2 5 5}]$} & & & poor condition, disintegrating. \\
$\mathbf{0 0 2 . 1 0 . 2 5 . 1}$ & 1 ?female (15) & (no data) & ('Euprymna berryi'). \\
{$[\mathbf{2 6 3 ]}$} & & & \\
\hline
\end{tabular}

*Note that the dimensions listed for the types of E. similis Sasaki, 1913, exclude all the specimens listed here from possible type material of E. similis.

Rossia pacifica Berry, 1911.

\begin{tabular}{llll}
\hline \multicolumn{1}{c}{ Reg. No. } & \multicolumn{1}{c}{ Specimen(s) } & \multicolumn{1}{c}{ Collection data } \\
\hline 996.3.15 & 1 male $(49)$, & Loc. [Hokkaido], Hakodate. & Poor condition. Syntypes of Rossia borealis \\
{$[\mathbf{0 5 2}]$} & 2 females $(63,46)$, & Coll. 21.i.1908 (M 41). & Sasaki, 1913c: 400 (also as a nomen nudum: \\
& 1 ? (42). & & Sasaki, 1913b: 247). Sa 57. '17'. \\
996.6.164.1 & 2 males (31, 31), & Loc. [Suruga Bay, Izu Peninsula, Shizuoka & Originally with 4 Sepiolina nipponensis. \\
{$[\mathbf{1 8 2 - 1 ]}$} & 1 ?female (42). & Pref.], Heda. Coll. Dec., 1936. & \\
\hline
\end{tabular}

Ref. Berry, 1911: 591; Sasaki, 1913c: 399; 1929: 154, text figs. 92-94, Pl. XVI figs. 3-6. Sasaki, 1929: 157, contrasted R. pacifica with $R$. borealis, concluding that the latter are aberrant forms of the same species.

Sepiola sp.

\begin{tabular}{cccc}
\hline Reg. No. & Specimen $(\mathrm{s})$ & Collection data & Comments \\
\hline $\mathbf{0 0 2 . 1 0 . 2 5 . 2}$ & 2 females $(15,15)$. & (no data) & ('Sepiola birostrata'). \\
{$[\mathbf{2 6 4}]$} & & & \\
\hline
\end{tabular}

Sepiolina nipponensis (Berry, 1911).

\begin{tabular}{clll}
\hline \multicolumn{1}{c}{ Reg. No. } & \multicolumn{1}{c}{ Specimen(s) } & \multicolumn{1}{c}{ Collection data } \\
\hline $\mathbf{9 9 6 . 6 . 1 6 4 . 2}$ & 2 males $(25,24)$, & Loc. [Shizuoka Pref.], Heda. & Originally with 3 Rossia pacifica. \\
{$[\mathbf{1 8 2 - 2}]$} & 2 females $(26,20)$. & Coll. Dec., 1936. & \\
\hline
\end{tabular}

cf. Sasaki, 1929: 149, text figs. 90, 91, Pl. XV figs. 15, 16a, b, 17, Pl. XVI figs. 1, 2. 


\section{Idiosepiidae}

Idiosepius paradoxus (Ortmann, 1888).

\begin{tabular}{|c|c|c|c|}
\hline Reg. No. & Specimen(s) & Collection data & Comments \\
\hline $\begin{array}{l}996.5 .73 \\
{[163]}\end{array}$ & $\begin{array}{l}11 \text { specs. } \\
\text { (largest } 14.5)\end{array}$ & $\begin{array}{l}\text { Loc. [Seto Sea, Hiroshima Pref.], Onomichi, } \\
\text { Koutajima. Coll. May, } 1906 \text { (M 39). }\end{array}$ & $\begin{array}{l}c f . \text { specimens listed by Sasaki, 1914a: } 600 \quad(6 \\
\text { specimens, Misaki; } 4 \text { specimens Inland [Seto] Sea). }\end{array}$ \\
\hline
\end{tabular}

cf. Sasaki, 1914a: 599; 1923: 213; 1929: 133, text figs. 78-79 (labelled Idiosepius pygmaeus), Pl. XV figs. 1-3.

\section{Sepiida: Sepiadariidae}

Sepiadarium kochii Steenstrup, 1881.

\begin{tabular}{|c|c|c|c|}
\hline Reg. No. & Specimen(s) & Collection data & Comments \\
\hline $\begin{array}{l}\text { 996.3.14 } \\
{[057]}\end{array}$ & 1 male $(15$, mature $)$ & $\begin{array}{l}\text { Loc. Suruga [Suruga Bay, Shizuoka Pref., } \\
\text { Numazu], Enoura. Coll. April } 1884 \text { (M 17)*, } \\
\text { Mitsukuri and Ishikawa. }\end{array}$ & $\begin{array}{l}\text { 'Sepiadarium sp.' Sa 51. cf. lot \#1 of Sasaki, 1914a: } \\
\text { 598. 'April, 1886'.* ‘16'. }\end{array}$ \\
\hline
\end{tabular}

002.10.24.1 3 males $(17,16,16)$ Loc. [Kyushu], Bungo [Oita Pref.], Beppu. $\quad$ Sa 53. Lot \#3 of Sasaki, $1914 a: 598$ [260] Coll. 13.iii.1899 (M 32), Terasaki.

cf. Sasaki, 1914a: 597; 1929: 152, Pl. XV figs. 18, 19. *Date incongruency: 'April, 1886' written in English on reverse of label but original Japanese script states 'Meiji 17' which was 1884.

\section{Sepiida: Sepiidae}

[Sasaki's 'DML' in sepiids excluded the rostrum in his paper of $1914 a$, p. 608, but measurements in the 1929 monograph included it]

Sepia andreana Steenstrup, 1875.

\begin{tabular}{cccc}
\hline Reg. No. & Specimen(s) & Collection data & \multicolumn{1}{c}{ Comments } \\
\hline $\mathbf{0 0 0 . 4 . 2 8 . 3 . 3}$ & 3 males $(91,86,85)$. & (no data) & From a mixed lot with 'Octopus' minor, S. cf. \\
{$[\mathbf{2 2 3}-3]$} & & & lycidas, Sepiella japonica and Loliolus japonicus. \\
\hline
\end{tabular}

cf. specimen DML 85mm of Sasaki, 1913a. Ref. Sasaki, 1913a: 75, Pl. II figs. 1-8; 1914a: 613; 1929: 196, Pl. I fig. 6, Pl. XVIII figs. 12, 13. The only species of Sepia found in Aomori Pref., northern Honshu. Compare with S. sasakii Wakiya and Ishikawa, 1921 ; see below.

Sepia appelloefi Wulker, 1910.

\begin{tabular}{llll}
\hline Reg. No. & \multicolumn{1}{c}{ Specimen(s) } & \multicolumn{1}{c}{ Collection data } & \multicolumn{1}{c}{ Comments } \\
\hline 996.5.130 & 1 male (78), & Loc. [Kyushu], Kagoshima. & $c f$. lot\#1 of Sasaki, 1913a, 1914a. Not illustrated. \\
{$[\mathbf{1 9 2}]$} & 1 female (54). & Coll. April, 1896 (M 29), Mitsukuri. & \\
\hline
\end{tabular}

Ref. Sasaki, 1913a: 82, text figs. 9, 10; 1914a: 618; 1929: 184, text fig. 106, Pl. XVII figs. 10, 11 a, b, 12.

Sepia aureomaculata Okutani and Horikawa in Okutani, Tagawa and Horikawa, 1987.

\begin{tabular}{|c|c|c|c|}
\hline Reg. No. & Specimen(s) & Collection data & Comments \\
\hline $\begin{array}{l}996.5 .131 \\
{[206]}\end{array}$ & $\begin{array}{l}1 \text { female (111, mature), } \\
1 \text { juv. (65). }\end{array}$ & $\begin{array}{l}\text { Loc. [Suruga Bay, Izu Peninsula, Shizuoka } \\
\text { Pref.], Heda. Coll. Dec., } 1936 .\end{array}$ & Ref. Okutani et al., 1987: 62, text figs. 16A-F. \\
\hline
\end{tabular}

Sepia erostrata Sasaki, 1929.

\begin{tabular}{llll}
\hline \multicolumn{1}{c}{ Reg. No. } & \multicolumn{1}{c}{ Specimen(s) } & \multicolumn{1}{c}{ Collection data } & \multicolumn{1}{c}{ Comments } \\
\hline 994.CD-05 & 1 mature male (43). & Loc. Sagami Bay, Manazuru. & Holotype by monotypy. Ref. Sasaki, 1929: 183, Pl. \\
[243] & & Coll. 23.iii.1915 (T 4), M. Sasaki. & XVII figs. 7a, b, 8, 9a-c. \\
\hline
\end{tabular}

Sepia esculenta Hoyle, 1885.

\begin{tabular}{|c|c|c|c|}
\hline Reg. No. & Specimen(s) & Collection data & Comments \\
\hline $\begin{array}{l}\text { 996.5.63.2 } \\
{[107-2]}\end{array}$ & 1 female (230). & $\begin{array}{l}\text { Loc. [Kyushu], Kagoshima. } \\
\text { Coll. Aug., } 1903 .\end{array}$ & In same lot as 3 Sepioteuthis lessoniana. \\
\hline $\begin{array}{l}996.6 .147 \\
{[208]}\end{array}$ & 1 female (148). & Loc. [Japan Sea, Kyoto Pref.], Miyazu Bay. & Sa 88. cf. lot \#2 of Sasaki, 1914a. \\
\hline $\begin{array}{l}996.6 .148 \\
{[209]}\end{array}$ & $\begin{array}{l}1 \text { male }(116), \\
1 \text { female }(108) \text {. }\end{array}$ & Loc. Yahata Bridge. & - \\
\hline $\begin{array}{l}\text { 996.6.149 } \\
{[214]}\end{array}$ & 2 males $(115,89)$. & $\begin{array}{l}\text { Loc. [Kanagawa Pref.], Misaki. } \\
\text { Coll. Dec., } 1908 .\end{array}$ & Sa 85 . \\
\hline $\begin{array}{l}996.6 .150 \\
{[202]}\end{array}$ & $\begin{array}{l}2 \text { females }(85,83), \\
3 \text { juv. }(44,41,32)\end{array}$ & (no data) & 'S1-S2 天 9-3 入港' \\
\hline
\end{tabular}


Sepia esculenta, continued

\begin{tabular}{|c|c|c|c|}
\hline Reg. No. & Specimen(s) & Collection data & Comments \\
\hline $\begin{array}{l}\text { 996.6.151 } \\
{[205]}\end{array}$ & 8 specs. $(53-83)$. & Loc. Misaki. & Sa 104. ('all young'). \\
\hline $\begin{array}{l}996.6 .152 \\
{[215]}\end{array}$ & 1 female (138). & $\begin{array}{l}\text { Loc. [Kyushu], Satsuma [Kagoshima Pref.], } \\
\text { Komenotsu. }\end{array}$ & Sa 89. \\
\hline $\begin{array}{l}996.6 .153 \\
{[203]}\end{array}$ & 2 specs. $(87,82)$. & Loc. Tokyo market. & - \\
\hline $\begin{array}{l}\text { 996.6.157 } \\
{[103]}\end{array}$ & 17 specs. $(72-170)$. & Loc. Tokyo market. & $\begin{array}{l}\text { Sa 84. cf. Sasaki, 1910: } 367 \text { and lot \#1 of Sasaki, } \\
\text { 1914a. }\end{array}$ \\
\hline
\end{tabular}

Ref. Sasaki, 1910: 367, Pl. 12 figs. 14A, B; Pl. 13 fig. 1; 1914a: 611; 1929: 175, Pl. 1 fig. 5, Pl. XVI figs. 15 a, b, $16,17$.

Sepia formosana Berry sensu Sasaki, 1929: 165.

\begin{tabular}{lccl}
\hline Reg. No. & Specimen(s) & Collection data & \multicolumn{1}{c}{ Comments } \\
\hline $\mathbf{0 0 1 . 1 0 . 4}$ & 3 ?males $(119,108,87)$. & (no data) & Regular large and small white spots; fin bars (cf. \\
{$[\mathbf{1 8 3}]$} & & & Sasaki, 1929, text fig. 165A). cf. S. subaculeata \\
& & (pars?). Loc. Taiwan?
\end{tabular}

cf. Sasaki, 1929: 165, text figs. 165A-E, 166, Pl. XXX figs. 9-11.

Sepia kobiensis andreanoides Hoyle sensu Sasaki, 1929.

\begin{tabular}{lccc}
\hline Reg. No. & Specimen(s) & Collection data & \multicolumn{1}{c}{ Comments } \\
\hline $\begin{array}{l}\text { 996.6.134 } \\
{[194]}\end{array}$ & (15 specs. (45-71). & 'hime kôika' [lesser cuttlefish]. Some specimens dissect- \\
ed. Body (sepion) rather broad $c f . S$. k. kobiensis.
\end{tabular}

cf. Sasaki, 1929: 206, text figs. 112-114, Pl. I fig. 7, Pl. XIX figs. 5, 6a, b, 7.

Sepia kobiensis beppuana Sasaki, 1929.

\begin{tabular}{|c|c|c|c|}
\hline Reg. No. & Specimen(s) & Collection data & Comments \\
\hline $\begin{array}{l}\text { 996.6.139 } \\
{[244]}\end{array}$ & $\begin{array}{l}7 \text { specs. }(57,56,54, \\
50,49,55,49) \text {. }\end{array}$ & $\begin{array}{l}\text { Loc. [Kyushu], Bungo, [Oita Pref.], Beppu. } \\
\text { Coll. 12.iii.1899 (M 32), Terasaki. }\end{array}$ & Syntypes. 2 sepions (one $79 \mathrm{~mm}$ long; other broken). \\
\hline $\begin{array}{l}002.10 .24 .2 \\
{[258]}\end{array}$ & 2 specs. $(18,16)$. & $\begin{array}{l}\text { Loc. [Kyushu], Oita [Pref.]. } \\
\text { Coll. 13.iii.1899, Terasaki. }\end{array}$ & $\begin{array}{l}\text { Syntypes. 'Sepia (Doratosepion) kobiensis.' cf. S. } \\
\text { kobiensis (juv.). 'teguri.' }\end{array}$ \\
\hline
\end{tabular}

Ref. Sasaki, 1929: 211, text fig. 116A-C, Pl. XIX figs. 13a, b, 14, 15.

Sepia kobiensis kobiensis Hoyle sensu Sasaki, 1929 (as Sepia kobiensis typicus Sasaki).

\begin{tabular}{lcll}
\hline Reg. No. & Specimen(s) & Collection data & \multicolumn{1}{c}{ Comments } \\
\hline $\begin{array}{l}\text { 996.6.137 } \\
{[196]}\end{array}$ & 15 specs. (44-64). & Loc. Tokyo market. & $\begin{array}{l}\text { Slimmer than specimens of } S . k . \text { andreanoides and } \\
\text { rostrum is more acutely pointed. }\end{array}$ \\
\hline
\end{tabular}

Ref. Sasaki, 1929: 204, text fig. 111, Pl. XIX figs. 1, 2, 3a-c, 4.

Sepia longipes Sasaki, 1913.

\begin{tabular}{llll}
\hline \multicolumn{1}{c}{ Reg. No. } & \multicolumn{1}{c}{ Specimen(s) } & \multicolumn{1}{c}{ Collection data } & \multicolumn{1}{c}{ Comments } \\
\hline $\mathbf{0 0 0 . 4 . 2 8 . 2}$ & 1 male (227). & Loc. [Chiba Pref. (Kazusa Prov.)], Chôshi. & ?Syntype. Accompanied by a small diagram. (cf. \\
[246] & & Coll. April, 1906 (M 39), Shimizu. & published date of six syntypes: 18.viii.1906; see text). \\
996.5.133 & 1 female (136). & Loc. Mikawa [Aichi Pref.], Mitani. & 43.1.25' (S 43 or M 43?). With two S. tenuipes. \\
{$\left[\begin{array}{l}\text { 207] } \\
\hline\end{array}\right.$} & & Coll. 25.i.1968? (1910?) & \\
\hline
\end{tabular}

Ref. Sasaki, 1913a: 78, Pl. 3, figs. 4, 5; 1914a: 619, Pl. XII figs. 4, 5; 1929: 188, text fig. 107, Pl. XVII fig. 13a, b.

Sepia lorigera Wülker, 1910.

\begin{tabular}{|c|c|c|c|}
\hline Reg. No. & Specimen(s) & Collection data & Comments \\
\hline $\begin{array}{l}\text { 996.6.158.1 } \\
{[104]}\end{array}$ & 1 male (204). & $\begin{array}{l}\text { Loc. 'Boshiu' [Chiba Pref.], Fuku-ura. } \\
\text { Coll. 15.x.1883. }\end{array}$ & $\begin{array}{l}\text { Labels '4659' 'near } S . \text { andreana' 'suji-ika.' Speci- } \\
\text { men is tagged '4659.' }\end{array}$ \\
\hline $\begin{array}{l}\text { 996.6.158.2 } \\
{[104]}\end{array}$ & $\begin{array}{l}2 \text { males }(200,144), \\
2 \text { females }(129,73) .\end{array}$ & Loc. Tokyo market (undated). & Sa 99. $c f$. lot \#1b of Sasaki, 1914a. \\
\hline $\begin{array}{l}996.6 .159 \\
{[105]}\end{array}$ & 3 males $(231,178,173)$ & $\begin{array}{l}\text { Loc. Sagami [Kanagawa Pref.], Misaki. } \\
\text { Coll. 7.v.1906 (M 39). }\end{array}$ & Sa 98. \\
\hline $\begin{array}{l}996.6 .160 \\
{[211]}\end{array}$ & $\begin{array}{l}1 \text { male }(158) \\
1 \text { female }(145)\end{array}$ & Loc. Tokyo market (undated). & $\begin{array}{l}\text { Sa 99. Includes a note that there are two lots labelled } \\
\text { 'No. 99.' }\end{array}$ \\
\hline
\end{tabular}

Arms 1 very long and slender in males (>2 times DML), with ends coiled and thickened. $c f$. Sasaki, 1913a: 77, text figs. 7, 8; 1914a: 619, 1929: 189, text fig. 108, Pl. XVII figs. 14a-c. 
Sepia cf. lycidas Gray, 1849.

\begin{tabular}{|c|c|c|c|}
\hline Reg. No. & Specimen(s) & Collection data & Comments \\
\hline $\begin{array}{l}000.4 .28 .3 .2 \\
{[223-2]}\end{array}$ & 2 females (both 92). & (no data) & $\begin{array}{l}\text { Originally in same lot with two 'O.' minor, four Loliolus } \\
\text { japonicus, three } S \text {. aculeata and one Sepiella japonica. }\end{array}$ \\
\hline
\end{tabular}

Arms subequal, ALO 4.3.2.1, fairly short, tapering rapidly, 4 sucker rows. Tentacle club with many very small suckers. Typical markings of S. lycidas not preserved (cf. Sepia subaculeata, below, and S. formosana, above).

Sepia madokai Adam, 1939 (nom. nov. for Sepia robsoni Sasaki, 1929; not S. robsoni Massy, 1927).

\begin{tabular}{|c|c|c|c|}
\hline Reg. No. & Specimen(s) & Collection data & Comments \\
\hline $\begin{array}{l}\text { 996.6.143 } \\
{[199]}\end{array}$ & $\begin{array}{l}2 \text { males }(\text { c. } 76,74), \\
1 \text { ?male }(40), 3 \text { ?females } \\
(80,74, \text { c. } 63), 1 \text { ? }(41)\end{array}$ & $\begin{array}{l}\text { Loc. Tokyo market. } \\
\text { Coll. Sept. (?) } 1885 \text { (sic). }\end{array}$ & $\begin{array}{l}\text { Syntypes. Sa 93. 'No. 4.' 'Sepia elliptica,' } \\
\text { 'Sepia torosa?' 'Sepia aculeata?' and (in Japa- } \\
\text { nese) 'hari-ika.' Sepions removed from all } \\
\text { specimens, but only } 5 \text { sepions now included. }\end{array}$ \\
\hline $\begin{array}{l}996.6 .140 \\
{[212]}\end{array}$ & $\begin{array}{l}2 \text { males }(90,87) \text {, } \\
2 \text { females }(73,64) \text {. }\end{array}$ & $\begin{array}{l}\text { Loc. [Kyushu], Kagoshima [Bay], Sakurajima, } \\
\text { off Kurokamicho. } \\
\text { Coll. 1.iv.1896 (M 29), Mitsukuri and Hara }\end{array}$ & $\begin{array}{l}\text { Sa 88. 'Sepia elliptica.' Loc. not listed by } \\
\text { Sasaki. }\end{array}$ \\
\hline $\begin{array}{l}996.6 .141 \\
{[198]}\end{array}$ & Sepion only (71). & Tokyo market. & 'Sepia elliptica.' (cf. specimens. in 996.6.143). \\
\hline $\begin{array}{l}996.6 .142 \\
{[213]}\end{array}$ & 1 male $(55)$ & $\begin{array}{l}\text { Loc. Sagami [Kanagawa Pref.], Misaki. Coll. } \\
\text { 14.ii.1891 (M 24), Fukuji Sakamoto [fide Ikeda]. }\end{array}$ & $\begin{array}{l}\text { Sa 97. 'Sepia elliptica.' '14/ II 24.' cf. S. ellip- } \\
\text { tica of Ikeda, 1891f: } 328 \text {. Not included by } \\
\text { Sasaki (1929). }\end{array}$ \\
\hline
\end{tabular}

Ref. Sasaki, 1929: 179, Pl. XVII figs. 2,3A-C (as Sepia robsoni); cf. Sepia elliptica Hoyle sensu Sasaki, 1914a: 612, Pl. XI figs. 11, 12; 1920: 192; not Sepia elliptica Hoyle, 1885: 189. See also Sasaki, 1910: 368, Pl. 13 figs. 4-8; Adam, 1939: 77; Khromov et al. $1998: 106$.

Sepia misakiensis Wülker, 1910.

\begin{tabular}{|c|c|c|c|}
\hline Reg. No. & Specimen(s) & Collection data & Comments \\
\hline $\begin{array}{l}\text { 996.6.145 } \\
{[178]}\end{array}$ & 1 spec. (66). & $\begin{array}{l}\text { Loc. [Kanagawa Pref.], Misaki. } \\
\text { Coll. Sasaki (no date given). }\end{array}$ & $\begin{array}{l}\text { Sa 106. } c f .67 \mathrm{~mm} \text { specimens of Sasaki, 1929: } \\
\text { 203; also, compare tentacle club in separate } \\
\text { container with Pl. XVIII fig. } 15 \text {. }\end{array}$ \\
\hline $\begin{array}{l}996.6 .146 \\
{[201]}\end{array}$ & 1 spec. (65). & $\begin{array}{l}\text { Loc. Misaki. } \\
\text { Coll. 5.viii.1902 (M 35), Mitsukuri. }\end{array}$ & $\begin{array}{l}\text { Sa 105. 'Shirasu.' Tentacles and left arm } 1 \\
\text { missing. }\end{array}$ \\
\hline
\end{tabular}

Both specimens listed by Sasaki, 1913a: 77, Pl. II fig. 16; 1914a: 617; 1929: 202, Pl. XVIII figs. 14, 15. Arms 1 decidedly longest, but still $<1 / 2$ DML. Posterior part of fins expanded into an auriculate lobe on each side. Body more slender than S. tokioensis. Tentacles very short, thicker than in S. tokioensis. Included by Khromov et al., 1998, as a junior synonym of S. tokioensis Ortmann, 1888. Identified here as two distinct species (see also Okutani et al., 1987).

Sepia pardex Sasaki, 1913.*

\begin{tabular}{llll}
\hline Reg. No. & \multicolumn{1}{c}{ Specimen(s) } & \multicolumn{1}{c}{ Collection data } & Comments \\
\hline 994.CD-02 & 1 male (231). & Loc. Boshu [Chiba Pref.], Katsuyama [Kyonan, & Holotype by monotypy [Sa] 101. This loc. listed \\
[242] & & Awakatsuyama]. Coll. 9.ii.1889. & under S. esculenta by Sasaki, 1910: 368. \\
\hline
\end{tabular}

Ref. Sasaki, 1913a: 74, Pl. III figs. 1-3. *Also identified as the junior objective synonym Sepia pardalis Sasaki, 1914a: 614, Pl. XII figs. 1-3; 1929: 187 (loc. transliterated as 'Kajiyama').

Sepia peterseni Appellöf, 1886.

\begin{tabular}{|c|c|c|c|}
\hline Reg. No. & Specimen(s) & Collection data & Comments \\
\hline $\begin{array}{l}996.6 .154 \\
{[098]}\end{array}$ & 14 males (84-104). & Loc. Tokyo market. & $\begin{array}{l}\text { Sa 70. cf. Sasaki, 1913a: } 82 \text { 'Tokyo fish market.' } \\
\text { Lot \#3 of Sasaki, 1914a. }\end{array}$ \\
\hline $\begin{array}{l}996.6 .155 \\
{[210]}\end{array}$ & 10 males (79-108). & $\begin{array}{l}\text { Loc. Mikawa [Aichi Pref.], Ise Bay. } \\
\text { Coll. 23.iv.1906. }\end{array}$ & Sa 71. Lot \#1 of Sasaki, 1914a. \\
\hline $\begin{array}{l}996.6 .156 \\
{[204]}\end{array}$ & 1 male (109). & $\begin{array}{l}\text { Loc. [Kyushu], Kagoshima market. } \\
\text { Coll. 29.iii.1906 (M 39), Mitsukuri and Hara. }\end{array}$ & Sa 72. Lot \#2 of Sasaki, 1914a. \\
\hline
\end{tabular}

Ref. Sasaki, 1914a: 618; 1929: 195, text fig. 109, Pl. XIII figs. 10, 11, Pl. XIX figs. 27a-c.

Sepia pharaonis Ehrenberg, 1831.

\begin{tabular}{llll}
\hline Reg. No. & \multicolumn{1}{c}{ Specimen(s) } & \multicolumn{1}{c}{ Collection data } \\
\hline $\mathbf{0 0 2 . 1 0 . 2 5 . 9}$ & 1 male (183). & Loc. 'Jolo' [Philippines?]. & cf. S. tigris Sasaki, 1929. 'Sepia torosa Ort.' \\
{$[\mathbf{0 9 4}]$} & & Coll. Jan., 1908 (M 41), Ijima. & \\
\hline
\end{tabular}

Compare Sasaki, 1929: 163, 165, 173. See also Adam and Rees, 1966: 22. (The Philippines specimen of $S$. torosa mentioned by Sasaki, 1929: 164, is in HUFM, catalogue ref. M-1144). 
Sepia sasakii Wakiya and Ishikawa, 1921 (S. andreana Steenstrup sensu Sasaki, 1913, by indication).

\begin{tabular}{lcll}
\hline Reg. No. & Specimen(s) & \multicolumn{1}{c}{ Collection data } & \multicolumn{1}{c}{ Comments } \\
\hline 996.6.135 & 6 males (77-91). & Loc. Boshu [Boso Peninsula, Chiba Pref.]. & Syntypes. Sa 89. 'Sepia andreana Stp.' (Col- \\
[195] & & cf. Loc. 'Awa' [Chiba Pref.]. Coll. 20.iv.1906 & lector fide Sasaki, 1913a: 75). \\
& & (M 39), [Watase].
\end{tabular}

Arms II stout and long. Sasaki (1929) did not mention this species, nor the paper of Wakiya and Ishikawa. Ref. Wakiya and Ishikawa, 1921: 290, Pl. II figs. 11a-c, indicating specimens identified as Sepia andreana Steenstrup sensu Sasaki, 1913a: 75, Pl. 2 figs 1-7 [figs 1-8?]. See also Sasaki, 1914a: 613 .

Sepia subaculeata Sasaki, 1913.

\begin{tabular}{llll}
\hline Reg. No. & \multicolumn{1}{c}{ Specimen(s) } & \multicolumn{1}{c}{ Collection data } & \multicolumn{1}{c}{ Comments } \\
\hline 997.3.1 & 2 females $(215,205)$, & Loc. Tokyo market [Nihonbashi fish market]. & Syntypes. Sa 102. cf. S. lycidas Gray. See also \\
[245] & 2 ?males $(102,93)$. & Coll. Nov., 1882 (M15). & Adam and Rees, 1966: 8, 10. \\
\hline
\end{tabular}

Ref. Sasaki, 1913a: 73, Pl. 3 figs. 6a, b; 1914a: 609, Pl. XII figs. 6a, b; 1929: 173, text fig. 104A-C, Pl. XVI figs. 13, 14a, b, Pl. XVII fig. 1.

Sepia tenuipes Sasaki, 1929 (Sepia andreanoides Hoyle sensu Sasaki, 1914).

\begin{tabular}{|c|c|c|c|}
\hline Reg. No. & Specimen(s) & Collection data & Comments \\
\hline $\begin{array}{l}\text { 996.6.136 } \\
{[181]}\end{array}$ & 2 males $(65,65)$. & Loc. Tokyo market. Coll. 'Sept., 1885.' & $\begin{array}{l}\text { ?Syntypes.* Sa 92. Fair condition-mutilated. 'Sepia } \\
\text { appellöfi.' ('Sepia longipes'). Sepions } 83,80 \mathrm{~mm} \text { long. }\end{array}$ \\
\hline $\begin{array}{l}996.5 .133 \\
{[207]}\end{array}$ & $\begin{array}{l}1 \text { female }(74) \text {, } \\
1 \text { ?male }(87) \text {. }\end{array}$ & $\begin{array}{l}\text { Loc. Mikawa [Aichi Pref.], Mitani. } \\
\text { Coll. 25.i.1910? }\end{array}$ & '43.1.25.' With a specimen of S. longipes. \\
\hline
\end{tabular}

*cf. lot \#1 of Sepia andreanoides Hoyle sensu Sasaki, 1914a: 614. Ref. Sasaki, 1929: 193, P1. XVIII figs. 1a, b, 2-8, 9a, b.

Sepia tokioensis Ortmann, 1888.

\begin{tabular}{llll}
\hline Reg. No. & \multicolumn{1}{c}{ Specimen(s) } & \multicolumn{1}{c}{ Collection data } \\
\hline 996.6.138 & 2 males $(69,67)$, & Loc. Tokyo market. & Sa 86. 'No. 7'. Incl. one dissected male. 'Sepia \\
{$[\mathbf{1 9 7 ]}$} & 1 female $(72)$, & & misakiensis figured by Sasaki, 1929' [comment \\
& 3 juv. $(45,41,32)$. & could not be verified]. \\
996.6.144 & 9 specs. (65-86). & [Tokyo Bay, Tokyo City] Gyotoku. & $\begin{array}{l}\text { Sa 87. Year fide Sasaki: Meiji 39 (first digit } \\
\text { missing on label with specimen). }\end{array}$ \\
\hline
\end{tabular}

Similar to S. misakiensis but body broader; tentacles longer and slimmer. Both lots listed by Sasaki, 1913a: 77; 1914a: 616. Ref. Sasaki, 1913a: 76, Pl. II figs. 9-15; 1929: 200, text fig. 110.

\begin{tabular}{|c|c|c|c|}
\hline Reg. No. & Specimen(s) & Collection data & Comments \\
\hline $\begin{array}{l}\text { 996.5.132 } \\
{[193]}\end{array}$ & 1 spec. (36). & $\begin{array}{l}\text { Loc. [Japan Sea, Toyama Bay, Toyama } \\
\text { Pref.], Namerikawa. Coll. 10.v.1905 (M 38). }\end{array}$ & cf. Sepia kobiensis toyamensis (too small to be a syntype). \\
\hline $\begin{array}{l}000.3 .31 .3 \\
{[221-2]}\end{array}$ & 1 set of beaks. & (no data) & 'S. acueata' (sic). With beaks of an 'Abraliopsis.' \\
\hline $\begin{array}{l}000.4 .28 .4 \\
{[224]}\end{array}$ & $\begin{array}{l}6 \text { specs. }(55,41 \text {, } \\
35,33, ?, ?) .\end{array}$ & $\begin{array}{l}\text { Loc. Tokyo market. } \\
\text { Coll. Nov., } 1885 \text { (M 18). }\end{array}$ & $\begin{array}{l}\text { 'akahari ika.' Very poor condition. Two specimens stuck } \\
\text { together (not measured). 'Sepia salcata Hoyle' 'S. } \\
\text { appellofi.' cf. S. appelloefi lot \#2 of Sasaki, 1914a: } 618 .\end{array}$ \\
\hline $\begin{array}{l}002.10 .24 .5 \\
{[257]}\end{array}$ & $\begin{array}{l}1 \text { ?female }(45) \\
2 ?(46,42)\end{array}$ & $\begin{array}{l}\text { Loc. Tokyo market. } \\
\text { Coll. Sept., 1885? (year ' } 18 \text { '). }\end{array}$ & $\begin{array}{l}\text { 'S. sulcata' 'S. kiensis' 'Sepia (Doratosepion) } \\
\text { kobiensis.' cf. S. sulcata lot \#1 of Ikeda, 1891f: } 328 .\end{array}$ \\
\hline $\begin{array}{l}002.10 .24 .6 \\
{[261]}\end{array}$ & 2 males $(59,49)$. & $\begin{array}{l}\text { Loc. [Chiba Pref.], Tateyama. } \\
\text { Coll. 28.v.1908 (M 41). }\end{array}$ & 'S. kiensis and S. kobiensis.' '41/5/28.' \\
\hline
\end{tabular}

Sepiella japonica Sasaki, 1929.

\begin{tabular}{|c|c|c|c|}
\hline Reg. No. & Specimen(s) & Collection data & Comments \\
\hline $\begin{array}{l}\text { 996.6.161 } \\
{[184]}\end{array}$ & $\begin{array}{l}1 \text { male }(142) \\
1 \text { female }(133)\end{array}$ & $\begin{array}{l}\text { Loc. [Tokyo Bay, Kanagawa Pref.,] Natagiri } \\
\text { [Yokosuka City, Natsujima-cho]. } \\
\text { Coll. 8.ii.1891 (M 24). }\end{array}$ & $\begin{array}{l}\text { Syntypes. Sa } 95 \text { '8/II 24' 'Sepiella maindroni.' cf. lot \#1 } \\
\text { of Ikeda, 1891f: } 330 \text {. }\end{array}$ \\
\hline $\begin{array}{l}996.6 .162 \\
{[185]}\end{array}$ & $\begin{array}{l}2 \text { males }(73,58), \\
3 \text { females } \\
(69,62,47)\end{array}$ & Loc. Tokyo market. Coll. 1.x.1885. & $\begin{array}{l}\text { Sa 96. 'Sepia inermis.' 'Sepiella maindroni.' cf. Ikeda lot } \\
\# 2 \text {. These specimens not mentioned or indicated by } \\
\text { Sasaki. }\end{array}$ \\
\hline $\begin{array}{l}000.4 .28 .3 .5 \\
{[223-5]}\end{array}$ & 1 female $(85)$ & (no data) & $\begin{array}{l}\text { From a mixed lot with 'Octopus' minor, three Sepia } \\
\text { andreana, two Sepia cf. lycidas and four Loliolus } \\
\text { japonicus. }\end{array}$ \\
\hline
\end{tabular}

Ref. Sasaki 1929: 219, text fig. 170, Pl. XVIII figs. 20-22, 23a, b, Pl. XIX fig. 28. See also 1913a: 85; 1914a: 621, text fig. 1 (as S. maindroni sensu Hoyle); Adam and Rees, 1966: 128, Pl. 39 figs. 228-231; cf. Sepia sinensis d'Orbigny, 1848: 289, and Sepiella maindroni sensu Hoyle, 1886: 149 (not Sepiella maindroni Rochebrune, 1884a: 89). 


\section{Decabrachia: Teuthida: Loliginidae}

(Genus group names follow Anderson, 2000).

Doryteuthis (Amerigo) pealei (Lesueur, 1821).

\begin{tabular}{llll}
\hline Reg. No. & \multicolumn{1}{c}{ Specimen(s) } & \multicolumn{1}{c}{ Collection data } & Comments \\
\hline $\begin{array}{l}\text { 996.5.107 } \\
{[129]}\end{array}$ & 1 female (165). & Loc. [Western North Atlantic, U.S.A.], & Sa 73. 'Loligo pealii.' \\
996.5.108 & 1 female (186). & Loc. [U.S.A., Massachusetts], Wood Hall & 'No. 38.' Sa 74. \\
{$[130]$} & & [Woods Hole] (undated). & \\
\hline
\end{tabular}

Heterololigo bleekeri (Keferstein, 1866).

\begin{tabular}{|c|c|c|c|}
\hline Reg. No. & Specimen(s) & Collection data & Comments \\
\hline $\begin{array}{l}\text { 996.5.120 } \\
{[131]}\end{array}$ & $\begin{array}{l}3 \text { females } \\
(181,165,146)\end{array}$ & (no data) & $\begin{array}{l}\text { Dissected, with stained viscera. Tentacles } \\
\text { very short. }\end{array}$ \\
\hline $\begin{array}{l}996.5 .123 \\
{[186]}\end{array}$ & $\begin{array}{l}1 \text { female } \\
\text { (193, immature) }\end{array}$ & Loc. [Kanagawa Pref.], Misaki. & Sa 65. Loc. listed by Sasaki, 1929: 127. \\
\hline $\begin{array}{l}996.5 .124 \\
{[187]}\end{array}$ & 1 male (155). & Loc. Tokyo market. & Sa 64. Loc. listed by Sasaki, 1929: 127. \\
\hline $\begin{array}{l}002.10 .25 .8 \\
{[265]}\end{array}$ & $\begin{array}{l}1 \text { male }(340), \\
3 \text { females } \\
(192,184,168)\end{array}$ & $\begin{array}{l}\text { Loc. [Kyushu], Satsuma, Kagoshima. } \\
\text { Coll. 30.iv.1906, Mitsukuri and Hara. }\end{array}$ & $\begin{array}{l}\text { Sa 62. Loc. \#2 of Sasaki, 1914a: } 605 . \\
\text { Includes a label 'Type specimen'(?)* }\end{array}$ \\
\hline
\end{tabular}

Ref. Sasaki, 1914a: 604; 1929: 125, text fig. 73, Pl. XIII figs. 6-8, 9a, b, 10 (as Loligo bleekeri). * These specimens have not been identified with any species other than $H$. bleekeri.

Loliolus (Nipponololigo) beka (Sasaki, 1929).

\begin{tabular}{|c|c|c|c|}
\hline Reg. No. & Specimen(s) & Collection data & Comments \\
\hline $\begin{array}{l}996.5 .105 \\
{[128]}\end{array}$ & $\begin{array}{l}16 \text { specs. } \\
(22-33, \text { juv. })\end{array}$ & $\begin{array}{l}\text { Loc. [Kyushu, Miyazaki Pref.], Ariake Sea. } \\
\text { Coll. Oct., } 1911 \text { (M 44), Tago. }\end{array}$ & $\begin{array}{l}\text { Syntypes. Sa 75; 'Loligo sumatrensis.' Two of these } \\
\text { specimens illustrated in Sasaki 1929, Pl. XIII figs. } \\
\text { 5a, b. cf. text p.122 (coll. 'Prof. Kishinoue'). }\end{array}$ \\
\hline $\begin{array}{l}996.5 .106 \\
{[125]}\end{array}$ & $\begin{array}{l}5 \text { females. (c. } 63,63 \text {, } \\
62,61,52) .\end{array}$ & Loc. Okayama market. Coll. 25.iv.1901. & $\begin{array}{l}\text { Syntypes. Sa 71; 'Loligo sumatrensis,' 'beka.' Listed } \\
\text { by Sasaki, 1914a: 603, and 1929: } 122 \text { (1 male is now } \\
\text { missing). }\end{array}$ \\
\hline $\begin{array}{l}996.5 .111 \\
{[119]}\end{array}$ & 1 female (73). & $\begin{array}{l}\text { Loc. [Kyushu, Nagasaki Pref.], Omura Bay. } \\
\text { Coll. Dec., } 1898 \text { (M 31). }\end{array}$ & Syntype. Sa 70 . \\
\hline $\begin{array}{l}996.5 .112 \\
{[236]}\end{array}$ & 2 females $(65,51)$. & $\begin{array}{l}\text { Loc. [Seto Sea, Okayama Pref.], Kojima } \\
\text { Bay. Coll. October, } 1903 \text { (M 36). }\end{array}$ & Syntypes. 'Loligo ? japonica.' \\
\hline
\end{tabular}

Ref. Sasaki, 1929: 121, text figs. 70A-C, 71, 72; Pl. XIII fig. 5a, b (as Loligo beka). Previously described as Loligo sumatrensis d'Orbigny sensu Sasaki, 1914a: 603-604 (cf. legends to text figs. 70-72 of Sasaki, 1929). See also Sasaki, 1910: 363, Pl. XII figs. 1-3.

Loliolus (Nipponololigo) japonicus (Hoyle, 1885).

\begin{tabular}{|c|c|c|c|}
\hline Reg. No. & Specimen(s) & Collection data & Comments \\
\hline $\begin{array}{l}\text { 996.5.109 } \\
{[134]}\end{array}$ & 7 specs. $(34-46)$ & Loc. Tokyo market. Coll. 1.x.1885. & $\begin{array}{l}\text { Sa 67. Sasaki, } 1914 a \text {, lot \#1. 'Loligo japonica } \\
\text { Stp.' 'Loligo sumatrensis.' cf. Ikeda, 1891a: } 28 .\end{array}$ \\
\hline $\begin{array}{l}996.5 .110 \\
{[136]}\end{array}$ & $\begin{array}{l}3 \text { females } \\
(79,72,70)\end{array}$ & $\begin{array}{l}\text { Loc. [Kanagawa Pref.], Sagami Bay. } \\
\text { Coll. 16.ii.1891. }\end{array}$ & Sa 68. Sasaki, $1914 a$, lot \#2. 'jindo ika 6' ' 8 ' \\
\hline $\begin{array}{l}996.5 .113 \\
{[132]}\end{array}$ & $\begin{array}{l}5 \text { specs. }(23,22,20 \\
18.5,18)\end{array}$ & $\begin{array}{l}\text { Loc. [Japan Sea], Tango Kuni [Kyoto Pref.], } \\
\text { Miyazu. Coll. 8.vii.1873 (M 6), Iizuka. }\end{array}$ & Sa 122. Loc. not listed by Sasaki. \\
\hline $\begin{array}{l}996.5 .126 .2 \\
{[189-2]}\end{array}$ & 2 males $(91,83)$ & (no data) & $\begin{array}{l}\text { 'S1-S2 天 9-3 入港' Originally with two } \\
\text { Uroteuthis edulis and one unidentified loliginid. }\end{array}$ \\
\hline $\begin{array}{l}000.3 .31 .4 .2 \\
{[222-2]}\end{array}$ & 1 female (68). & $\begin{array}{l}\text { Loc. [Ishikawa Pref.], Togi. } \\
\text { Coll. 24.ix.1927, Uchida. }\end{array}$ & $\begin{array}{l}\text { Specimen not seen by Sasaki. ('jindo ika') } \\
\text { Originally in lot with one A. fangsiao. }\end{array}$ \\
\hline $\begin{array}{l}000.4 .28 .3 .4 \\
{[223-4]}\end{array}$ & $\begin{array}{l}1 \text { male }(59) \\
3 \text { females } \\
(65,53,51)\end{array}$ & (no data) & $\begin{array}{l}\text { From a mixed lot with 'Octopus' minor, Sepiella } \\
\text { japonica, three Sepia andreana and two Sepia } \\
\text { cf. lycidas. }\end{array}$ \\
\hline $\begin{array}{l}000.4 .28 .5 \\
{[216]}\end{array}$ & 10 specs. $(31-51)$. & Coll. May, 1902 (M 35), Kuma. & - \\
\hline $\begin{array}{l}002.12 .4 .3 \\
{[259]}\end{array}$ & 1 female (62). & Loc. Shimane Pref. Coll. 10.xi.1906 (M 39). & Sa 69. 'Loligo japonica'. Sasaki, 1914a, lot \#3. \\
\hline
\end{tabular}

Ref. Sasaki, 1914a: 602; 1929: 112, text figs. 60A, B, 61A-C, 62, Pl. XIV figs. 1-6 (as Loligo japonica). See also Sasaki, $1909: 485$. 
Loliolus (Nipponololigo) uyii (Wakiya and Ishikawa, 1921).

\begin{tabular}{lccc}
\hline Reg. No. & Specimen(s) & Collection data & Comments \\
\hline $\mathbf{9 9 7 . 7 . 2}$ & 1 ?female (50). & (no data) & With one large nudibranch gastropod. '25' \\
{$[\mathbf{1 7 7 ]}$} & & & \\
\hline
\end{tabular}

cf. Wakiya and Ishikawa, 1921: 286, Pl. II figs. 12a-h (as Loligo uyii).

Uroteuthis edulis edulis (Hoyle, 1885) (originally as Loligo edulis communis Sasaki, 1929).

\begin{tabular}{|c|c|c|c|}
\hline Reg. No. & Specimen(s) & Collection data & Comments \\
\hline $\begin{array}{l}\text { 996.5.115 } \\
{[121]}\end{array}$ & $\begin{array}{l}11 \text { males }(62-92) \text {, } \\
7 \text { females }(55-89) \text {. }\end{array}$ & (no data) & 'Loligo edulis.' \\
\hline $\begin{array}{l}996.5 .122 \\
{[097]}\end{array}$ & $\begin{array}{l}3 \text { males }(254,218,188) \text {, } \\
5 \text { females }(111-173) .\end{array}$ & (no data) & $\begin{array}{l}\text { cf. Sasaki, 1914a, lot \#4 (L. kensaki?). Two } \\
\text { notes about catching squid, Mr. Kuma, and } \\
\text { vernacular name 'mehikari' (cf. Loligo edulis: } \\
\text { Okutani et al., 1987: 99). }\end{array}$ \\
\hline $\begin{array}{l}996.5 .125 \\
{[188]}\end{array}$ & 1 female (320). & (no data) & ('Loligo edulis, kensaki ika'). \\
\hline $\begin{array}{l}\text { 996.5.126.1 } \\
{[189-1]}\end{array}$ & $\begin{array}{l}1 \text { male }(151), \\
1 \text { female }(149) .\end{array}$ & (no data) & 'S1-S2 天 9-3 入港' \\
\hline
\end{tabular}

Ref. Sasaki, 1914a: 601-602 (incl. syntypes of Uroteuthis kensaki; see below); 1929: 107, text figs. 57, 58, Pl. XIII figs. 1, 2, 3a, b.

Uroteuthis kensaki (Wakiya and Ishikawa, 1921) (Loligo edulis Hoyle sensu Sasaki, 1914).

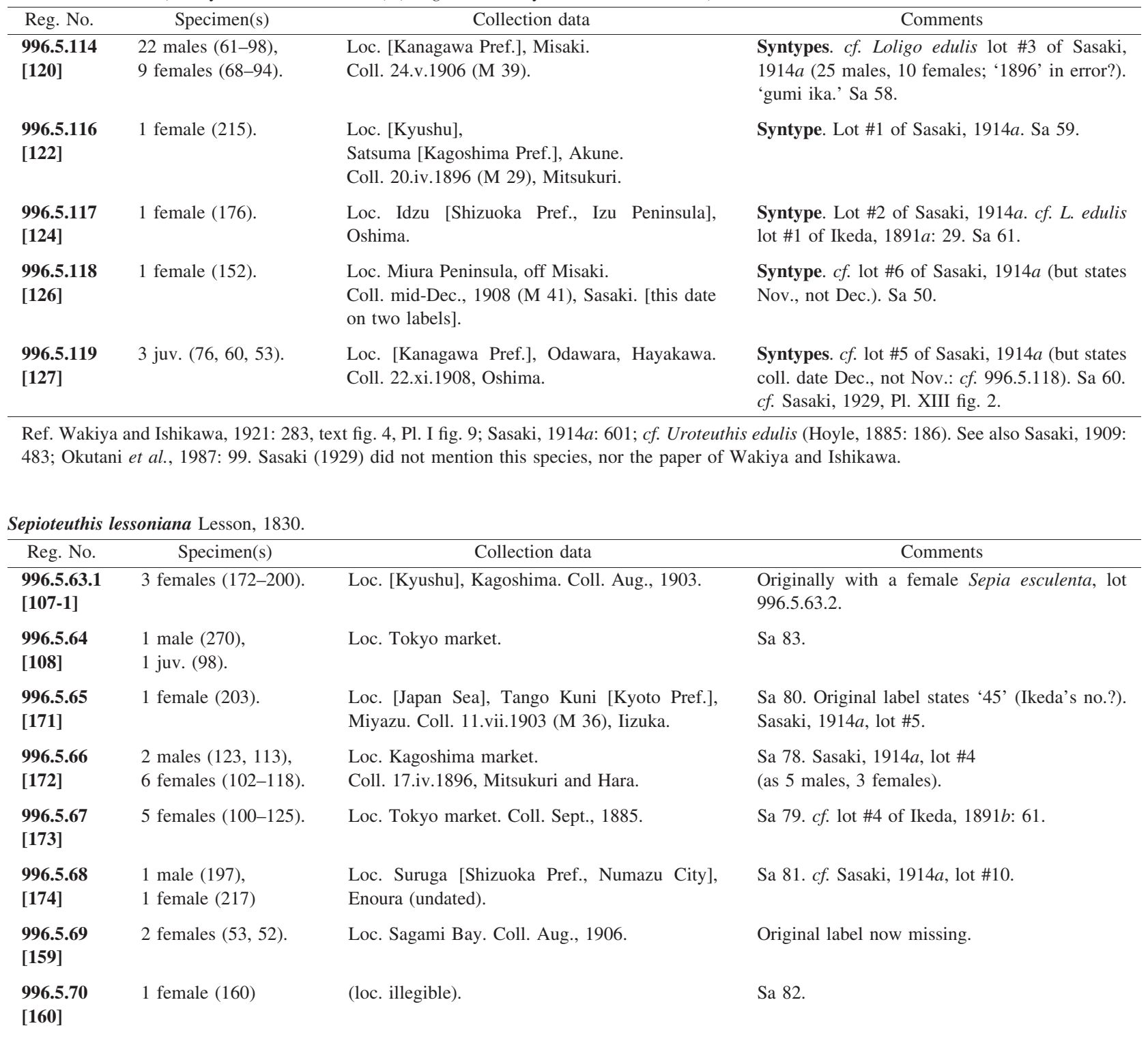


Sepioteuthis lessoniana, continued

\begin{tabular}{|c|c|c|c|}
\hline Reg. No. & Specimen(s) & Collection data & Comments \\
\hline $\begin{array}{l}996.5 .71 \\
{[161]}\end{array}$ & 1 spec. (77). & Loc. Tokyo market (undated). & Sa 100. 'aori ika 2.' \\
\hline $\begin{array}{l}996.5 .72 \\
{[162]}\end{array}$ & 1 male (107). & $\begin{array}{l}\text { Loc. Idzu [Izu, Shoto Islands], } \\
\text { Oshima. Coll. April, } 1887 \text { (M 20). }\end{array}$ & $\begin{array}{l}\text { Sa 90. } c f . \text { lot \#6 of Ikeda, } 1891 b: 62 \text { (TL 230). } \\
\text { Sasaki, } 1914 a \text {, lot \#2. }\end{array}$ \\
\hline $\begin{array}{l}996.5 .74 \\
{[164]}\end{array}$ & 1 juv. (48). & Coll. $1960 ?$ & “'60-1063” \\
\hline $\begin{array}{l}996.5 .75 \\
{[165]}\end{array}$ & 4 juv. $(42,34,33,26)$ & (no data) & - \\
\hline $\begin{array}{l}996.5 .128 \\
{[190]}\end{array}$ & 1 female $(215)$ & (no data) & - \\
\hline $\begin{array}{l}996.5 .129 \\
{[191]}\end{array}$ & 1 female (105). & (no data) & ‘S1-S2 天 9-13 入港’ \\
\hline $\begin{array}{l}996.6 .163 \\
{[112]}\end{array}$ & $\begin{array}{l}1 \text { male }(250), \\
2 \text { females }(214,185), \\
\text { all mature. }\end{array}$ & $\begin{array}{l}\text { Loc. [Kanagawa], Misaki. } \\
\text { Coll. Aug., } 1906 \text { (M 39). }\end{array}$ & Sa 76. Sasaki, $1914 a$, lot \#6. \\
\hline $\begin{array}{l}000.3 .31 .2 \\
{[220]}\end{array}$ & Beaks only. & (no data) & $\begin{array}{l}\text { Two sets of beaks 'Sepioteuthis-large' and } \\
\text { 'E. morsei-small'. }\end{array}$ \\
\hline $\begin{array}{l}000.4 .28 .1 \\
{[113]}\end{array}$ & 1 male $(350)$. & $\begin{array}{l}\text { Loc. [Japan Sea, Toyama Pref.], } \\
\text { Namerikawa. }\end{array}$ & $\begin{array}{l}\text { Sasaki, } 1914 a \text {, lot \#3. Sa 77. cf. lot \#1 of Ikeda, } \\
\text { 1891b: } 61 .\end{array}$ \\
\hline \multicolumn{4}{|c|}{ Ref. Sasaki, 1910: 365; 1914a: 606-607; 1929: 127, text figs. 74-76A, B, 77, P1. XIV figs. 15-17, P1. XXIX figs. 8, 9.} \\
\hline \multicolumn{4}{|c|}{ Unidentified loliginids. } \\
\hline Reg. No. & Specimen(s) & Collection data & Comments \\
\hline $\begin{array}{l}\text { 996.5.121 } \\
{[135]}\end{array}$ & 2 females $(97,89)$. & Loc. Taiwan, Kiirun [Chi-lung]. & '27.' (wooden tag.). ('Loligo sibogae Adam, 1954'). \\
\hline $\begin{array}{l}996.5 .126 .3 \\
{[189-3]}\end{array}$ & 1 male $(70)$. & (no data) & $\begin{array}{l}\text { 'S1-S2 天 9-3 入港' Originally with one U. edulis } \\
\text { and two L. japonicus. }\end{array}$ \\
\hline $\begin{array}{l}996.5 .127 \\
{[180]}\end{array}$ & 1 spec. (41). & (no data) & ('Loligo (Photololigo) edulis'). \\
\hline $\begin{array}{l}001.10 .2 .5 \\
{[137]}\end{array}$ & 1 male $(85)$ & (no data) & $\begin{array}{l}\text { Coll. 1960? ('Loligo edulis'). Originally with an } \\
\text { Amphioctopus cf. kagoshimensis, one M. loennbergii } \\
\text { and two chiroteuthids. }\end{array}$ \\
\hline $\begin{array}{l}001.10 .3 \\
{[133]}\end{array}$ & 1 female (101). & (no data) & cf. Uroteuthis edulis. ('Loligo sp.' [foreign origin]). \\
\hline $\begin{array}{l}002.12 .4 .2 \\
{[262]}\end{array}$ & 2 specs. $(25,23)$ & $\begin{array}{l}\text { Loc. [Kanagawa Pref.], Odawara. } \\
\text { Coll. 4.vi.1891 (M 24), Ishida. }\end{array}$ & - \\
\hline
\end{tabular}

\section{Enoploteuthidae}

Watasenia scintillans (Berry, 1911).

\begin{tabular}{|c|c|c|c|}
\hline Reg. No. & Specimen(s) & Collection data & Comments \\
\hline $\begin{array}{l}996.5 .85 \\
{[152]}\end{array}$ & $\begin{array}{l}30 \text { females (48-61, } \\
\text { mature with eggs). }\end{array}$ & $\begin{array}{l}\text { Loc. [Japan Sea] Etchu [Toyama Pref.], } \\
\text { Namerikawa. Coll. May, } 1905 \text { (M 38). }\end{array}$ & - \\
\hline $\begin{array}{l}996.5 .86 \\
{[153]}\end{array}$ & $\begin{array}{l}106 \text { specimens } \\
(36-45)\end{array}$ & $\begin{array}{l}\text { Loc. [Kanagawa Pref.], Odawara. } \\
\text { Coll. early March, } 1907 \text { (M 40). }\end{array}$ & $\begin{array}{l}\text { Sa 31. 'beni ika'* [red squid]. ' } 87 \text { females'; '17 [19] } \\
\text { females' separate within a large glass vial. Lot \#4 of } \\
\text { Sasaki, 1914b: } 88-89 \text {; lot \#4, 1916: } 95 \text {. }\end{array}$ \\
\hline $\begin{array}{l}996.5 .87 \\
{[154]}\end{array}$ & $\begin{array}{l}14 \text { females (54-61, } \\
\text { mature with eggs). }\end{array}$ & Loc. Namerikawa. Coll. May, 1905. & Arm-tip photophores removed from 4 specimens. \\
\hline $\begin{array}{l}996.5 .88 \\
{[155]}\end{array}$ & 78 specs. $(36-42)$. & $\begin{array}{l}\text { Loc. Boshu, Awa [Chiba Pref.], Shirahama, } \\
\text { Banda. Coll. 9.ii.1908 (M 41). }\end{array}$ & $\begin{array}{l}\text { Sa 33. A label by Sasaki notes that he sent three } \\
\text { specimens to a Dr. Mortensen, } 15 \text { July, 1914. Lot \#1, } \\
1914 b \text {; lot } \# 5,1916 \text {. }\end{array}$ \\
\hline $\begin{array}{l}996.5 .89 \\
{[138]}\end{array}$ & 1 ? female $(60)$ & (no data) & Arm-tip photophores removed. Very poor condition. \\
\hline $\begin{array}{l}996.5 .90 \\
{[139]}\end{array}$ & $\begin{array}{l}27 \text { females (52-61, } \\
\text { mature with eggs). }\end{array}$ & $\begin{array}{l}\text { Loc. Etchu, Namerikawa. Coll. 22.v.1905 } \\
\text { (M 38), Watase. }\end{array}$ & - \\
\hline
\end{tabular}


Watasenia scintillans, continued

\begin{tabular}{|c|c|c|c|}
\hline Reg. No. & Specimen(s) & Collection data & Comments \\
\hline $\begin{array}{l}996.5 .91 \\
{[140]}\end{array}$ & $\begin{array}{l}28 \text { females (49-63, } \\
\text { mature with eggs) }\end{array}$ & $\begin{array}{l}\text { Loc. Namerikawa. } \\
\text { Coll. 22.v.1905, Watase. }\end{array}$ & - \\
\hline $\begin{array}{l}996.5 .92 \\
{[148]}\end{array}$ & $\begin{array}{l}19 \text { females (50-61, } \\
\text { mature with eggs). }\end{array}$ & (no data) & 'pereni' (in katakana script). \\
\hline $\begin{array}{l}996.5 .93 \\
{[149]}\end{array}$ & $\begin{array}{l}24 \text { females (50-62, } \\
\text { mature with eggs). }\end{array}$ & $\begin{array}{l}\text { Loc. Namerikawa. } \\
\text { Coll. 25.v.1905, Watase. }\end{array}$ & Arm-tip photophores removed from 4 specimens. \\
\hline $\begin{array}{l}996.5 .94 \\
{[150]}\end{array}$ & $\begin{array}{l}22 \text { females (40-45, } \\
\text { mated, with maturing } \\
\text { eggs). }\end{array}$ & $\begin{array}{l}\text { Loc. [Kanagawa Pref.], Odawara. } \\
\text { Coll. mid-March (no year given). }\end{array}$ & $\begin{array}{l}\text { Sa } 35 \text {. 'mariaged female } 22 \text { ' }[\text { sic }] \text {. Arm-tip photo- } \\
\text { phores removed from } 1 \text { specimen. Lot \#5b, 1914b; } \\
\text { lot \#6, } 1916 \text {. }\end{array}$ \\
\hline $\begin{array}{l}996.5 .95 \\
{[151]}\end{array}$ & $\begin{array}{l}16 \text { specs. } \\
(35-44, \text { immature })\end{array}$ & $\begin{array}{l}\text { Loc. Boshu [Chiba Pref.], } \\
\text { NishiMisaki. Coll. 16.ii.1891 (M 24). }\end{array}$ & $\begin{array}{l}\text { Sa 32. cf. Onychoteuthis sp. of Ikeda, 1891d: } 241 . \\
\text { Sasaki W. scintillans lot \#3, 1914b; lot \#1, } 1916 .\end{array}$ \\
\hline $\begin{array}{l}996.5 .96 \\
{[156]}\end{array}$ & $\begin{array}{l}7 \text { females (49-57, } \\
\text { mature with eggs). }\end{array}$ & Loc. Taiwan, Chi-lung. & Arm-tip photophores removed from 6 specimens. \\
\hline $\begin{array}{l}996.5 .97 \\
{[157]}\end{array}$ & $\begin{array}{l}17 \text { females (52-60, } \\
\text { mature with eggs). }\end{array}$ & Loc. Namerikawa. Coll. May, 1905. & $\begin{array}{l}\text { All bar one have had most or all arm-tip photophores } \\
\text { removed (cut). The eyes of } 6 \text { specimens have been } \\
\text { dissected or removed. }\end{array}$ \\
\hline $\begin{array}{l}996.5 .98 \\
{[158]}\end{array}$ & $\begin{array}{l}20 \text { females (53-61, } \\
\text { mature with eggs). }\end{array}$ & (no data) & $\begin{array}{l}9 \text { specimens have had one or more arm-tip photo- } \\
\text { phores removed. }\end{array}$ \\
\hline $\begin{array}{l}996.5 .99 \\
{[114]}\end{array}$ & $\begin{array}{l}8 \text { females }(53-60 \\
\text { mature with eggs })\end{array}$ & (no data) & $\begin{array}{l}\text { 'Abraliopsis sp.' Arm-tip photophores removed (cut) } \\
\text { from one specimen. }\end{array}$ \\
\hline $\begin{array}{l}996.5 .100 \\
{[115]}\end{array}$ & $\begin{array}{l}2 \text { females }(59,58, \\
\text { mature with eggs })\end{array}$ & (no data) & - \\
\hline $\begin{array}{l}996.5 .101 \\
{[116]}\end{array}$ & $\begin{array}{l}10 \text { males }(35-40) \\
1 \text { female }(44)\end{array}$ & Loc. Odawara. & Sa 35. 'Lot 3.' Lot \#5a, 1914b; lot \#6, 1916. \\
\hline $\begin{array}{l}996.5 .102 \\
{[117]}\end{array}$ & $\begin{array}{l}5 \text { specs. }(41,39,37, \\
36,35 \text {, immature })\end{array}$ & $\begin{array}{l}\text { Loc. Boshu, Awa [Tochigi Pref.], } \\
\text { Tateyama Bay, Hazama Village. } \\
\text { Coll. mid-Feb., 1907, Oka. }\end{array}$ & Sa 36. Lot \#2, 1914b; lot \#3, 1916. \\
\hline $\begin{array}{l}996.5 .103 \\
{[118]}\end{array}$ & $\begin{array}{l}1 \text { female }(61 \text {, mature } \\
\text { with eggs). }\end{array}$ & (no data) & - \\
\hline $\begin{array}{l}996.5 .104 \\
{[123]}\end{array}$ & $\begin{array}{l}3 \text { females }(62,60,58, \\
\text { mature with eggs })\end{array}$ & (no data) & Arm-tip photophores removed from one specimen. \\
\hline $\begin{array}{l}002.12 .5 .1 \\
{[256]}\end{array}$ & $\begin{array}{l}1 \text { female }(51 \text {, mature } \\
\text { with eggs). }\end{array}$ & $\begin{array}{l}\text { Loc. Etchu [Toyama Pref.], Uozu. Coll. } \\
\text { 24.v.1905, Watase. }\end{array}$ & Sa 34 . \\
\hline
\end{tabular}

Ref. Sasaki, 1914b: 75, text fig. 2, Pl. I figs. 1-5, Pl. II figs. 1-12, Pl. III figs. 1-6; 1916: 94; 1929: 249, text fig. 124, Pl. XXI figs. 9, 10. *Vernacular name most commonly 'hotaru ika' [firefly squid]: 'beni ika' or 'aka ika' [red squid] refers to redness of digestive gland showing through transparent mantle of fresh specimens (Sasaki, 1914b: 81). Photophore removals and eye dissections attributable to research by Watase.

\begin{tabular}{lccc} 
?Abraliopsis sp. & & \\
\hline Reg. No. & Specimen(s) & Collection data & Comments \\
\hline $\mathbf{0 0 0 . 3 . 3 1 . 3}$ & 1 set of beaks & (no data) & Beaks only. 'Abraliopsis' 'S. acueata' (sic). \\
{$[\mathbf{2 2 1 - 1 ]}$} & & & With beaks of Sepia sp. \\
\hline
\end{tabular}

\section{Octopoteuthidae}

Octopoteuthis sicula Rüppell, 1844.

\begin{tabular}{|c|c|c|c|}
\hline Reg. No. & Specimen(s) & Collection data & Comments \\
\hline $\begin{array}{l}\text { 996.6.165 } \\
\text { [179] }\end{array}$ & 1 female $(22)$ & $\begin{array}{l}\text { Loc. [?Chiba Pref.], Tateyama. } \\
\text { [Coll. April, 1896; Prof. Oka]. }\end{array}$ & ' 85 ' The single specimen mentioned by Sasaki. \\
\hline
\end{tabular}

Ref. Sasaki, 1916: 96; 1929: 256, text fig. 125A, B, Pl. XXI figs. 17-19. 


\section{Onychoteuthidae}

Moroteuthis loennbergii Ishikawa and Wakiya, 1914.

\begin{tabular}{|c|c|c|c|}
\hline Reg. No. & Specimen(s) & Collection data & Comments \\
\hline $\begin{array}{l}\text { 996.5.61 } \\
{[169]}\end{array}$ & 1 spec. (180). & Loc. Sagami Bay, Misaki.* Coll. June, 1895. & Sa 29. \\
\hline $\begin{array}{l}\text { 996.5.61A } \\
{[170]}\end{array}$ & 1 spec. (c. 200). & Loc. Sagami Bay, Misaki.* Coll. 11.viii.1896. & Sa 30 . \\
\hline $\begin{array}{l}001.10 .2 .1 \\
{[063]}\end{array}$ & 1 spec. (166). & Coll. $1960 ?$ & $\begin{array}{l}\text { ' ' } 60-355 \text { ' (= Lot \#355, coll. 1960?). Originally } \\
\text { with one A. cf. kagoshimensis. }\end{array}$ \\
\hline
\end{tabular}

Ref. Ishikawa and Wakiya, 1914a: 445, Pl. XLV fig. 1, Pl. XLVI figs. 2a, b, 3-8; See also Sasaki, 1916: 91; Sasaki, 1929: 235, text fig. 119, Pl. XX figs. 13a, b; Kubodera et al. 1998: 283. *See Introduction.

\section{Histioteuthidae}

Histioteuthis hoylei (Goodrich, 1896).

\begin{tabular}{|c|c|c|c|}
\hline Reg. No. & Specimen $(\mathrm{s}) *$ & Collection data & Comments \\
\hline $\begin{array}{l}996.5 .54 \\
{[095]}\end{array}$ & 1 male (172). & (no data) & $\begin{array}{l}\text { cf. Histioteuthis sp. of Ikeda, 1891d: } 240 . \\
\text { cf. lot \#2 of Sasaki, } 1916 .\end{array}$ \\
\hline $\begin{array}{l}996.5 .55 \\
{[064]}\end{array}$ & 1 female (68). & Loc. Sagami Bay. & $\begin{array}{l}\text { Sa 26. 'Histioteuthis dofleini Pfeffer.' 'Calliteu- } \\
\text { this reversa' 'Histioteuthis reversa' 'Stigmato- } \\
\text { teuthis dofleini Pfeffer.' Lot \#3 of Sasaki, } 1916 .\end{array}$ \\
\hline $\begin{array}{l}996.5 .56 \\
{[067]}\end{array}$ & 1 male (140). & $\begin{array}{l}\text { Loc. } 36^{\circ} 14^{\prime} \mathrm{N} ., 142^{\circ} 18^{\prime} \text { E., off Ibaraki [Pref.]. } \\
\text { Coll. 15.vi.1904, S. Takahashi. }\end{array}$ & $\begin{array}{l}\text { 'Stigmatoteuthis dofleini Pfeffer.' Sa 24. In } \\
\text { stomach contents of a sperm whale. Lot \#1 of } \\
\text { Sasaki, } 1916 .\end{array}$ \\
\hline
\end{tabular}

Ref. Sasaki, 1916: 98; 1929: 258, text figs. 126A-F, 127, Pl. XXII figs. 1-3 (as Stigmatoteuthis dofleini); Voss et al., 1998: 315. * Sexes fide Sasaki, 1916.

Histioteuthis meleagroteuthis (Chun, 1910).

\begin{tabular}{llll}
\hline Reg. No. & Specimen(s) & \multicolumn{1}{c}{ Collection data } & \multicolumn{1}{c}{ Comments } \\
\hline 994.CD-01 & 1 spec. (31). & Loc. Sagami Bay, Miura, Misaki, Uchibata, & Holotype by monotypy of Meleagroteuthis \\
[248] & & c. $400 \mathrm{fm}$. [731.5 m]. Coll. 4.iii.1898, (M 31) Kuma. & separata Sasaki, 1915. Sa 27. \\
\hline
\end{tabular}

Ref. Sasaki, 1915: 131, text figs. 1, 2; 1916: 103; 1929: 262, Pl. XXII figs. 4, 5, 6a, b. Identified as an aberrant Histioteuthis meleagroteuthis by Voss et al., 1998: 353 (cf. Voss, 1969: 804, 809).

\section{Ommastrephidae}

Eucleoteuthis luminosa (Sasaki, 1915).

\begin{tabular}{|c|c|c|c|}
\hline Reg. No. & Specimen(s) & Collection data & Comments \\
\hline $\begin{array}{l}997.3 .2 \\
{[240]}\end{array}$ & $\begin{array}{l}1 \text { male }(139), 1 \text { ?female }(130) \text {, } \\
7 \text { females }(151,151,150,149, \\
137,119,117)\end{array}$ & $\begin{array}{l}\text { Loc. Sagami Bay, } 6 \text { miles }[11.1 \mathrm{~km}] \text { off } \\
\text { Misaki, } 700 \mathrm{fm}[1280 \mathrm{~m}] . \\
\text { Coll. 5.viii.1906, Watase. }\end{array}$ & $\begin{array}{l}\text { Syntypes. Sa 12. 'Symplectoteuthis luminosa' } \\
\text { 'suji-ika.' Originally with a specimen of } \\
\text { E. berryi: see } 997.3 .3 \text {. cf. Sasaki's '10' syntypes. }\end{array}$ \\
\hline $\begin{array}{l}996.5 .16 \\
{[086]}\end{array}$ & $\begin{array}{l}1 \text { male (148), } 1 \text { juv. }(117), \\
4 \text { dissected: } 121 \text { (female), } 135 \text {, } \\
132,123 \text {. }\end{array}$ & $\begin{array}{l}\text { Loc. [Kanagawa Pref.], Odawara. } \\
\text { Coll. Sept. }\end{array}$ & $\begin{array}{l}\text { No original label found. } 4 \text { specimens dissected } \\
\text { extensively. }\end{array}$ \\
\hline $\begin{array}{l}996.5 .17 \\
{[087]}\end{array}$ & 1 male (166). & Loc. Tokyo market (undated). & Sa 13 . \\
\hline $\begin{array}{l}996.5 .18 \\
{[088]}\end{array}$ & 3 females $(165,158,156)$ & Coll. 1906 (M 39). No other data. & $\begin{array}{l}\text { One specimen dissected; its abdominal contents } \\
\text { missing. }\end{array}$ \\
\hline $\begin{array}{l}996.5 .19 \\
{[089]}\end{array}$ & 3 females $(168,152,147)$ & Loc. Misaki. Coll. Aug., 1906. & - \\
\hline $\begin{array}{l}996.5 .20 \\
{[079]}\end{array}$ & $\begin{array}{l}3 \text { males }(161,138,129), \\
1 \text { female }(152)\end{array}$ & Loc. Misaki. Coll. Aug., 1906. & - \\
\hline $\begin{array}{l}996.5 .22 \\
{[081]}\end{array}$ & 1 male (126), 1 female (128). & $\begin{array}{l}\text { Loc. [Kanagawa Pref.], off Odawara. } \\
\text { Coll. 17.ix.1917. }\end{array}$ & 'suji ika.' \\
\hline $\begin{array}{l}996.5 .23 \\
{[068]}\end{array}$ & 1 ?male (135). & (no data) & Partially dissected abdomen and arms. \\
\hline $\begin{array}{l}996.5 .24 \\
{[069]}\end{array}$ & 1 ?female (120, immature). & $\begin{array}{l}\text { Loc. [Kanagawa Pref.], Misaki. } \\
\text { Coll. Aug., } 1906 \text { (M 39). }\end{array}$ & - \\
\hline
\end{tabular}


Eucleoteuthis luminosa, continued

\begin{tabular}{|c|c|c|c|}
\hline Reg. No. & Specimen(s) & Collection data & Comments \\
\hline $\begin{array}{l}\text { 996.5.25 } \\
{[106]}\end{array}$ & 8 specs. $(118-168)$ & Loc. Misaki Coll. Aug., 1906. & $\begin{array}{l}\text { (includes } 1 \text { specimen of L. japonicus, DML } \\
78 \mathrm{~mm}) .\end{array}$ \\
\hline $\begin{array}{l}996.5 .26 \\
{[070]}\end{array}$ & 3 males $(149,133,128)$ & $\begin{array}{l}\text { Loc. [Sagami Bay, Shizuoka Pref.], off Atami. } \\
\text { Coll. 23.vi.1905, Mr. Din [Dean]. }\end{array}$ & $\begin{array}{l}\text { All partially dissected with one or more ventral } \\
\text { arms removed. }\end{array}$ \\
\hline $\begin{array}{l}996.5 .27 \\
{[071]}\end{array}$ & $\begin{array}{l}2 \text { males }(140, ?), \\
2 \text { females }(145,116)\end{array}$ & Coll. 5.viii.1906 (Loc. not given). & $\begin{array}{l}\text { 'Towers friend' 'suji ika'. Cut into head, body } \\
\text { and tail pieces. Body of one male missing. }\end{array}$ \\
\hline $\begin{array}{l}996.5 .28 \\
{[072]}\end{array}$ & 1 male (head width 28 ). & (no data) & $\begin{array}{l}\text { Head with arms and tail (with fin) only. The eye } \\
\text { membranes have been dissected. }\end{array}$ \\
\hline $\begin{array}{l}996.5 .60 \\
{[093]}\end{array}$ & $\begin{array}{l}3 \text { males }(170,147,131) \\
6 \text { females }(128-148)\end{array}$ & Loc. Sagami Bay, Misaki. Coll. Aug., 1906. & - \\
\hline \multirow{2}{*}{\multicolumn{4}{|c|}{$\begin{array}{l}\text { Ref. Sasaki, 1915: 144, text fig. 4, Pl. IV figs. 7-13; 1916: 106; 1929: 293, text fig. 140a-d, Pl. XXIV figs. 4a, b, } 5 \text { (as Symplectoteuthis luminosa). } \\
\text { Type species of Eucleoteuthis Berry, } 1916 \text { (cf. Wormuth, 1998: 379). }\end{array}$}} \\
\hline & & & \\
\hline Reg. No. & Specimen(s) & Collection data & Comments \\
\hline $\begin{array}{l}996.5 .29 \\
{[073]}\end{array}$ & 2 specs. $(173,160)$ & Loc. U.S.A., Massachusetts. & Sa 3. \\
\hline $\begin{array}{l}996.5 .30 \\
{[074]}\end{array}$ & 3 specs. $(207,205,203)$. & $\begin{array}{l}\text { Loc. [U.S.A.], Massachusetts, Cape Cod. } \\
\text { Coll. B.H. van Vleek. }\end{array}$ & Sa 2. \\
\hline $\begin{array}{l}996.5 .62 \\
{[111]}\end{array}$ & 6 specs. $(214-220)$ & (no data) & Sa 1. \\
\hline
\end{tabular}

Ommastrephes bartramii (Lesueur, 1821).

\begin{tabular}{llll}
\hline Reg. No. & \multicolumn{1}{c}{ Specimen(s) } & \multicolumn{1}{c}{ Collection data } & \multicolumn{1}{c}{ Comments } \\
\hline $\mathbf{9 9 6 . 5 . 5 3}$ & 2 specs. (245, 240, & Loc. Sagami [Kanagawa Pref.], & Sa 11. 'Sthenoteuthis bartrami' 'baka ika' \\
[110] & immature). & Odawara. Coll. Nov., 1885. & 'medama aka ika' [red-eyed squid]. \\
\hline
\end{tabular}

Ref. Sasaki, 1916: 105; 1929: 289, text figs. 139a-h, Pl. I fig. 8, Pl. XXIV figs. 1-3, as Stenoteuthis bartrami (sic). cf. O. inisignis of Ikeda, 1891c: 146, and the lot examined by Sasaki, 1916: 106.

Ornithoteuthis volatilis (Sasaki, 1915).

\begin{tabular}{|c|c|c|c|}
\hline Reg. No. & Specimen(s) & Collection data & Comments \\
\hline $\begin{array}{l}\text { 994.CD-04 } \\
\text { [239] }\end{array}$ & $\begin{array}{l}1 \text { male }(204) \\
1 \text { female }(200)\end{array}$ & $\begin{array}{l}\text { Loc. Sagami Bay, [Shizuoka Pref.], } \\
\text { Off Atami. Coll. 24.vi.1906 (M 39), Aoki. }\end{array}$ & Syntypes. Sa 9. Lot \#1 of Sasaki, 1915. \\
\hline $\begin{array}{l}996.5 .32 \\
{[061]}\end{array}$ & 1 spec. (143). & $\begin{array}{l}\text { (Loc. not stated). } \\
\text { Coll. 29.ix.1905, Mr. Dean. }\end{array}$ & Syntype. $c f$. the male in Sasaki's lot \#2. \\
\hline $\begin{array}{l}996.5 .33 \\
{[065]}\end{array}$ & $\begin{array}{l}2 \text { females (both } 137 \text {, } \\
\text { with eggs). }\end{array}$ & Loc. off Atami. Coll. 23.ix.1905, Mr. Dean. & $\begin{array}{l}\text { Syntypes. The females of Sasaki lot \#2. Label } \\
\text { signed 'Kumakichi'. } \\
\text { Observed 'flying' at the surface. }\end{array}$ \\
\hline
\end{tabular}

Ref. Sasaki, 1915: 138, text fig. 3, Pl. IV figs. 1, 2, 3a-c, 4a, b, 5, 6. See also 1916: 105; 1929: 281, text figs. 135a-e, 136a-f, Pl. XXIII figs. 7-10, $11 \mathrm{a}, \mathrm{b}$, (as Ommastrephes volatilis). Type species of Ornithoteuthis Okada, 1927a ( $f$. Adam, 1957; Dunning, 1998). These are the original five 'type' specimens listed by Sasaki, 1915. (No holotype was designated, despite the assertions of Dunning, 1998: 429).

Sthenoteuthis oualaniensis (Lesson, 1830).

\begin{tabular}{|c|c|c|c|}
\hline Reg. No. & Specimen(s) & Collection data & Comments \\
\hline $\begin{array}{l}\text { 996.5.31 } \\
{[062]}\end{array}$ & $\begin{array}{l}5 \text { females }(149,145, \\
140,140,135)\end{array}$ & Loc. Tokyo market (undated). & $\begin{array}{l}\text { 'Ommastrephes oualaniensis' All specimens immature. } \\
\text { cf. Ommastrephes sp. lot \#2 of Ikeda, 1891c: } 147 .\end{array}$ \\
\hline $\begin{array}{l}996.5 .58 \\
{[059]}\end{array}$ & 1 juv. (50). & (no data) & - \\
\hline
\end{tabular}

cf. Sasaki, 1929: 296, text figs. 176-177, 178a-h, Pl. XXX fig. 8, based on specimens from Okinawa and Formosa [Taiwan]. See also Dunning, 1998: 425 . 
Todarodes pacificus pacificus (Steenstrup, 1880).

\begin{tabular}{|c|c|c|c|}
\hline Reg. No. & Specimen(s) & Collection data & Comments \\
\hline $\begin{array}{l}\text { 996.5.21 } \\
{[080]}\end{array}$ & $\begin{array}{l}3 \text { specs. } \\
(172,135, ?)\end{array}$ & (no data) & $\begin{array}{l}\text { One specimen head and arms only. ('Eucleoteuthis } \\
\text { luminosa'). }\end{array}$ \\
\hline $\begin{array}{l}996.5 .34 \\
{[066]}\end{array}$ & $\begin{array}{l}3 \text { females } \\
(196,190,178)\end{array}$ & (no data) & $\begin{array}{l}\text { Sa 6. 'Ommastrephes sloanei pacificus.' Contorted } \\
\text { and tied together with string. }\end{array}$ \\
\hline $\begin{array}{l}996.5 .35 \\
{[090]}\end{array}$ & 2 females $(180,172)$. & Loc. Tokyo market. Coll. Nov. '85 [1885]. & Sa 5. \\
\hline $\begin{array}{l}996.5 .36 \\
{[091]}\end{array}$ & 1 female (195). & $\begin{array}{l}\text { Loc. [Shikoku], Tosa [Kochi Pref.]. } \\
\text { Coll. } 1905 \text { (M 38), Tago. }\end{array}$ & Sa 7. 'surume ika' 'female.' \\
\hline $\begin{array}{l}996.5 .37 \\
{[082]}\end{array}$ & 1 female (188). & $\begin{array}{l}\text { Loc. Misaki. Coll. 9.viii.1897, } 130 \text { fa. } \\
\text { [238 m], Aoki. [Sasaki's label, in English]. }\end{array}$ & $\begin{array}{l}\text { Sa 8. Another label, in Japanese, states: Loc. } \\
\text { Okayama market, Coll. 9.viii.1905 (M 38), Kuma. }\end{array}$ \\
\hline $\begin{array}{l}996.5 .38 \\
{[083]}\end{array}$ & 3 specs., in pieces. & Loc. Tokyo market. Coll. Nov., 1882. & Sa 4. Heads, arms and viscera missing. \\
\hline $\begin{array}{l}996.5 .39 \\
{[084]}\end{array}$ & 1 female, head only & (no data) & - \\
\hline $\begin{array}{l}996.5 .40 \\
{[085]}\end{array}$ & 1 female $(250)$ & $\begin{array}{l}\text { Loc. Miura, Mitani [Misaki?]. } \\
\text { Coll. 25.i.1910 (M 43). }\end{array}$ & - \\
\hline $\begin{array}{l}996.5 .41 \\
{[092]}\end{array}$ & $\begin{array}{l}1 \text { male }(169) \\
1 \text { female }(178)\end{array}$ & $\begin{array}{l}\text { Loc. Misaki Mar. Exptl. Stn. } \\
\text { Coll. 28.xii.1904, Iizuka and Tsuchida. }\end{array}$ & $\begin{array}{l}\text { Another label, in Japanese, states: Tokyo market, } \\
\text { Coll. 27.iv.1904. (M 37). }\end{array}$ \\
\hline
\end{tabular}

\section{Thysanoteuthidae}

Thysanoteuthis rhombus Troschel, 1857.

\begin{tabular}{lcll}
\hline Reg. No. & Specimen(s) & \multicolumn{1}{c}{ Collection data } & \multicolumn{1}{c}{ Comments } \\
\hline 996.5.52 & 1 spec. (170). & Loc. Sagami [Kanagawa Pref.], \\
[075] & & off Atami. Coll. 23.ix.1905. & Sa 14. Lot \#1 of Sasaki, 1916. \\
\hline
\end{tabular}

Ref. Sasaki, 1916: 107; 1929: 301, text fig. 141, Pl. XXIV figs. 6-8.

\section{Chiroteuthidae}

Chiroteuthis (Chirothauma) imperator (Chun, 1908).

\begin{tabular}{|c|c|c|c|}
\hline Reg. No. & Specimen(s) & Collection data & Comments \\
\hline $\begin{array}{l}\text { 996.5.76 } \\
{[166]}\end{array}$ & 1 spec., in pieces. & $\begin{array}{l}\text { Loc. Sagami Bay, Yodomi. } \\
\text { Coll. 1.v.1899, } 350 \text { fa. }[640 \mathrm{~m}] \text {, Kuma. }\end{array}$ & $\begin{array}{l}\text { Sa 15. Includes intact tentacle club. Lot \#4 of Sasaki, } \\
1916 .\end{array}$ \\
\hline $\begin{array}{l}996.5 .77 \\
{[167]}\end{array}$ & 1 female (190). & $\begin{array}{l}\text { Loc. [Kanagawa Pref.], Misaki. } \\
\text { Coll. 24.ix.1908. }\end{array}$ & $\begin{array}{l}\text { Sa 16. Condition quite good. Includes one tentacle. } \\
\text { Sasaki lot } \# 5 \text {. }\end{array}$ \\
\hline $\begin{array}{l}996.5 .78 \\
{[141]}\end{array}$ & $\begin{array}{l}3 \text { specs. } \\
(145,140,118)\end{array}$ & $\begin{array}{l}\text { Loc. Sagami Kuni [Kanagawa Pref.], Odawara. } \\
\text { Coll. 1.v.1891 (M 24). }\end{array}$ & Sa 17. Sasaki lot \#2. \\
\hline $\begin{array}{l}996.5 .79 \\
{[142]}\end{array}$ & 2 specs. $(130,100)$ & Loc. Miura, Misaki. Coll. Sept., 1887. & $\begin{array}{l}\text { Sa 18. Sasaki lot \#1. cf. Chiroteuthis veranyi } \\
\text { specimens of Ikeda, } 1894 c: 44 .\end{array}$ \\
\hline $\begin{array}{l}996.5 .80 \\
{[143]}\end{array}$ & 2 specs. $(150,110)$ & $\begin{array}{l}\text { Loc. Soshu [Kanagawa Pref.], Misaki. } \\
\text { Coll. 27.viii.1891 (M 24). }\end{array}$ & Sa 19. Sasaki lot \#3. \\
\hline $\begin{array}{l}996.5 .81 \\
{[144]}\end{array}$ & 1 spec. (117) & (no data) & Sa 20. \\
\hline $\begin{array}{l}996.5 .82 \\
{[145]}\end{array}$ & 1 spec. (76). & (no data) & Sa 21. \\
\hline $\begin{array}{l}996.5 .83 \\
{[146]}\end{array}$ & 1 spec. $(76)$ & $\begin{array}{l}\text { Loc. Suruga Bay [Shizuoka Pref.]. Coll. } \\
\text { 28.viii.? }\end{array}$ & 'No. 1' (not Sasaki's: Sa 1 is an Illex illecebrosus). \\
\hline $\begin{array}{l}996.5 .84 \\
{[147]}\end{array}$ & 1 spec. (146). & Coll. 3.ii.1983 (?). & No original label. \\
\hline
\end{tabular}

Ref. Sasaki, 1916: 108; 1929: 305, text fig. 142A-D, Pl. XXIV figs. 9, 10M, R. 
Chiroteuthis sp.

\begin{tabular}{lccc}
\hline Reg. No. & Specimen & Collection data & \multicolumn{1}{c}{ Comments } \\
\hline $\begin{array}{l}\text { 001.10.2.3 } \\
\text { [076] }\end{array}$ & 1 spec. (122). & (no data) $60-355$ ' [Lot \#355, coll. 1960?]. Originally with an \\
001.10.2.4 & 1 female (117). & (no data) cf. kagoshimensis, a loliginid and 001.10.2.4. \\
[077] & & & (ditto, included with 001.10.2.3), 'yûrei ika.' \\
\hline
\end{tabular}

\section{Mastigoteuthidae}

Idioteuthis (Idioteuthis) latipinna Sasaki, 1916.

\begin{tabular}{lclc}
\hline Reg. No. & Specimen(s) & Collection data & Comments \\
\hline 994.CD-03 & 1 spec. (238). & $\begin{array}{l}\text { Outside of Okinose bank [Sagami Bay] from a depth } \\
\text { of about } 400 \mathrm{fa} \text { [732 m]. Coll. 20.iv.1906, K. Aoki. }\end{array}$ & Holotype by monotypy. Sa 23. \\
[233] & & & \\
\hline
\end{tabular}

Ref. Sasaki, 1916: 108, Pl. III figs. 1-5; and 1929: 312, text fig. 143A, B (as Mastigoteuthis latipinna). Type species of Idioteuthis Sasaki, 1916.

See also Salcedo-Vargas and Okutani, 1994: 124.

\section{Cranchiidae}

Cranchia scabra Leach, 1817.

\begin{tabular}{llll}
\hline Reg. No. & Specimen(s) & \multicolumn{1}{c}{ Collection data } & \multicolumn{1}{c}{ Comments } \\
\hline 994.CD-06 & 1 spec. (55). & Loc. [Kyushu, Kagoshima Pref.], Osumi, & cf. Sasaki, 1929: 331, Pl. XXVI fig. 13a, b. \\
[217] & & Kimotsuki-gun, Sata-mura. Coll. Jan., 1910 & ('same-hada hozuki ika'). \\
& & $(M$ 43), Tago. & \\
\hline
\end{tabular}

Ref. Sasaki, 1929: 329, text figs. 151A, B, 152, 153a-c, Pl. XXVI figs. 13a, b, 14, 15.

Leachia pacifica (Issel, 1908).

\begin{tabular}{lccc}
\hline Reg. No. & Specimen(s) & Collection data & \\
\hline $\mathbf{0 0 4 . 8 . 2}$ & 1 spec. (46). & Loc. Misaki. (undated) & 'Leachia pacifica Issel' (old label, from Meiji \\
{$[\mathbf{2 5 4}]$} & & & period, in ink). ('tougata ika'). \\
\hline
\end{tabular}

Ref. Sasaki, 1929: 338, text figs. 159A, B, Pl. XXVII figs. 7a, b, 8-15, 16a, b, 17 (as Pyrgopsis pacificus).

Liocranchia reinhardtii (Steenstrup, 1856).

\begin{tabular}{llll}
\hline Reg. No. & Specimen(s) & \multicolumn{1}{c}{ Collection data } & \multicolumn{1}{c}{ Comments } \\
\hline 996.5.57 & 2 specs. (23, 13). & Loc. [Kanagawa Pref.] Aburatsubo (surface). & Dated 26.xii.1894 by Sasaki, 1916. ('hôzuki \\
[060] & & Coll. 26.xii.1904 (M 37). & ika'). \\
\hline
\end{tabular}

Ref. Sasaki, 1916: 112. See also Sasaki, 1929: 332, text figs. 154, 155A, B, 156, 157A, B, 158A-C, Pl. XXVI figs. 16a, b, Pl. XXVII figs. 1a, b, $2-4$.

Teuthowenia elongata Sasaki, 1929.

\begin{tabular}{lccc}
\hline Reg. No. & Specimen(s) & Collection data & \multicolumn{1}{c}{ Comments } \\
\hline 996.5.50 & (missing) & Loc. Misaki. & Holotype by monotypy. 'Teuthowenia elongata \\
{$[\mathbf{2 3 4}]$} & & n.sp.' Empty jar. \\
\hline
\end{tabular}

Ref. Sasaki, 1929: 327, Pl. XXVI figs. 7-12.

Teuthowenia tagoi Sasaki, 1929.

\begin{tabular}{llll}
\hline Reg. No. & Specimen(s) & \multicolumn{1}{c}{ Collection data } & \multicolumn{1}{c}{ Comments } \\
\hline 996.5.51 & 1 spec. (18) & Loc. [Suruga Bay], Suruga Prov. [Shizuoka & Holotype by monotypy. Specimen badly con- \\
[235] & & Pref., Numazu City], Enoura. Coll. 3.iii.1902 & voluted. Collector's name is from old jar label: \\
& & (M 35), 'shirasu' net, Mr. Nomura. & $\begin{array}{l}\text { Sasaki stated that Tago was the collector (hence } \\
\text { the species name). }\end{array}$ \\
\hline
\end{tabular}

Ref. Sasaki, 1929: 326, text fig. 150A, B, P1. XXVI figs. 1a, b, 2-5, 6a, b.

\section{REFERENCES}

Adam, W. (1939), “Cephalopoda. Part II. II. Révision des espèces Indo-Malaises du genre Sepia Linné, 1758,” Siboga-Expeditie, 55b: 34-92.

Adam, W. (1941), "Notes sur les céphalopodes. XVI Sur une nouvelle espèce de céphalopode (Octopus robsoni sp. nov.) de la mer rouge," Bullétin du Musée Royal d'Histoire Naturelle de Belgique, 17: 1-5.

Adam, W. (1954), "Cephalopoda Part III. IV Céphalopodes à l'exclusion des genres Sepia, Sepiella et Sepioteuthis," SibogaExpeditie, 55c: $121-198$.

Adam, W. (1957), "Notes sur les céphalopodes. XXIII. Quelques espèces des Antilles," Bulletin de l'Institut Royal des Sciences Naturelles de Belgique, 33 (7): 1-10. 
Adam, W., and Rees, W. J. (1966), “A review of the cephalopod family Sepiidae," John Murray Expedition 1933-34, Scientific Reports, 11, Trustees of the British Museum (Natural History), London, pp. 1-165.

Alvarino, A., and Hunter, T. R. (1981), "New records of Alloposus mollis Verrill (Cephalopoda; Octopoda) from the Pacific Ocean," The Nautilus, 95: 26-32.

Anderson, F. E. (2000), "Phylogenetic relationships among loliginid squids (Cephalopoda: Myopsida) based on analyses of multiple data sets," Zoological Journal of the Linnean Society, 130: 603-633.

Appellöf, A. (1886), "Japanska Cephalopoder," Kungliga Svenska Vetenkaps-akademiens Handlingar, 21 (13): 1-40.

Berry, S. S. (1911), "Preliminary notices of some new Pacific cephalopods," Proceedings of the United States National Museum, 40 (1838): 589-592.

Berry, S. S. (1916), "Cephalopoda of the Kermadec Islands," Proceedings of the Academy of Natural Sciences of Philadelphia, $\mathbf{6 8}$ 45-66, Pl. VI-IX.

de Blainville, H. M. D. (1823), "Memoire sur les espèces du genre calmar (Loligo, Lamarck)," Journal de Physique, de Chimie et d'Histoire naturelle, 96: 116-135.

Chun, C. (1908), "Über Cephalopoden der Deutschen Tiefsee-Expedition,” Zoologischer Anzeiger, 33 (2): 86-89.

Chun, C. (1910), "Die Cephalopoden, I. Oegopsida," in Wissenschaftliche Ergebnisse der Deutschen Tiefsee-Expedition auf dem Dampfer 'Valdivia' 1898-1899, 18, Chun, C., Ed., Gustav Fischer, Jena, pp. 1-401, 63 plates.

Cuvier, G. L. C. (1797), Tableau élémentaire de l'histoire naturelle des animaux, Paris, p. 710, 14 plates.

Dunning, M. C. (1998), "A review of the systematics, distribution, and biology of the arrow squid genera Ommastrephes Orbigny, 1835, Sthenoteuthis Verrill, 1880, and Ornithoteuthis Okada, 1927 (Cephalopoda: Ommastrephidae)," Smithsonian Contributions to Zoology, 586: 425-433.

Ehrenberg, C. G. (1831), "Cephalopoda in mare rubro viventia." in Animalia invertebrata exclusis insectes. Symbolae physicae, seu icones et descriptiones Corparum Naturalium novorum aut minus cognitorum, quae ex itineribus per Libyam, Aegyptum, Nubiam, Dongalam, Syriam, Arabiam et Habessiniam, Pars zoologica, Hemprich, P. C., and Ehrenberg, C. G., Ed., Berlin, Vol. 4: 6 unnumbered pages.

Eydoux, J. F. T., and Souleyet, F. L. (1852), "Mollusques céphalopodes” in Zoologie, Vol. 2, Libraire de la Société de Géographie, Paris, pp. 7-36.

Gleadall, I. G. (1993), "Identification of the long-ligula octopuses of Japan: a status report," in Recent Advances in Cephalopod Fisheries Biology, Okutani, T., O’Dor, R. K., and Kubodera, T., Ed., Tokai University Press, Tokyo, pp. 145-158.

Gleadall, I. G. (1997), "Hong Kong cephalopods: a brief review of current knowledge and identification of specimens collected in 1995," in The Marine Flora and Fauna of Hong Kong and Southern China, Vol. IV, Morton, B., Ed., Hong Kong University Press, Hong Kong, pp. 503-513.

Gleadall, I. G. (2002), "The pseudophallus of the incirrate Octopoda: an organ specialized for releasing spermatophores singly," Abhandlungen der Geologischen Bundesanstalt, Wien, 57: 69-78.

Gleadall, I. G. (2003a), "A note on the Cephalopoda type specimens in the Zoology Department of Tokyo University Museum," Journal of Molluscan Studies, 69 (4): 375-380.

Gleadall, I. G. (2003b), “Asian species of the genus Amphioctopus,” Zoological Science, 20 (12): 1529.

Gleadall, I. G. (2004), "Some old and new genera of octopus," Interdisciplinary Information Sciences, 10: 99-112.

Goodrich, E. S. (1896), "Report on a collection of Cephalopoda from the Calcutta Museum," Transactions of the Linnean Society of London, Zoology (Ser. 2), 7: 1-24.

Gray, J. E. (1849), Catalogue of the Mollusca in the collection of the British Museum, Part I. Cephalopoda antepedia, British Museum, London, p. 164.

Hoyle, W. E. (1885), "Diagnoses of new species of Cephalopoda collected during the cruise of H. M. S. 'Challenger.'-Part II. The Decapoda." Annals and Magazine of Natural History, Ser. 5, 16: 181-203.

Hoyle, W. E. (1886), "Report on the Cephalopoda collected by H. M. S. Challenger during the years 1873-76," Report on the Scientific Results of the Voyage of H. M. S. Challenger during the years 1873-76, Zoology, 16: 1-245, London, Edinburgh, H.M.S.O.

Ijima, I., and Ikeda, S. (1895a), "Kikei no tako (Opisthoteuthis depressa n. sp.) ni tsuite" [On a deformed octopus, Opisthoteuthis depressa, n. sp.], Dôbutsugaku Zasshi [Zoological Magazine, Tokyo], 7: 211-222, 3 textfigs, (in Japanese; publ. 20 July, 1895).

Ijima, I., and Ikeda, S. (1895b), "Description of Opisthoteuthis depressa, n. sp.," Journal of the College of Science of Imperial University, Tokyo, 8 (2): 323-337, Pl. XXXIII figs. 1-9 (publ. 19 Dec., 1895).

Ijima, I., and Ikeda, S., (1902), "Notes on a specimen of Amphitretus obtained in the Sagami Sea," Nihon Dôbutsugaku Iho [Annotationes Zoologicae Japonenses], 4: 85-101, Pl. 2.

Ikeda, S. (1890a), "A list of Japanese Cephalopoda in the Zoological Institute of Imperial University [Tokyo]," Dôbutsugaku Zasshi [Zoological Magazine, Tokyo], 2 (25): 479-482 (in Japanese).

Ikeda, S. (1890b), "Rika daigaku hyôhon nihon tôsokurui mokuroku (dai ni kai)" [List of specimens of Japanese Cephalopoda at the Science College [Tokyo] (part 2)], Dôbutsugaku Zasshi [Zoological Magazine, Tokyo], 2 (26): 526-532 (in Japanese).

Ikeda, S. (1891a), "Rika daigaku hyôhon nihon tôsokurui mokuroku (dai san kai)" [List of specimens of Japanese Cephalopoda at the Science College [Tokyo] (part 3)], Dôbutsugaku Zasshi [Zoological Magazine, Tokyo], 3: 23-30 (in Japanese).

Ikeda, S. (1891b), "Rika daigaku hyôhon nihon tôsokurui mokuroku (dai yon kai)" [List of specimens of Japanese Cephalopoda at the Science College [Tokyo] (part 4)], Dôbutsugaku Zasshi [Zoological Magazine, Tokyo], 3: 60-63 (in Japanese).

Ikeda, S. (1891c), "Rika daigaku hyôhon nihon tôsokurui mokuroku (dai go kai)" [List of specimens of Japanese Cephalopoda at the Science College [Tokyo] (part 5)], Dôbutsugaku Zasshi [Zoological Magazine, Tokyo], 3: 144-147 (in Japanese).

Ikeda, S. (1891d), "Rika daigaku hyôhon nihon tôsokurui mokuroku (dai rokkai)" [List of specimens of Japanese Cephalopoda at the Science College [Tokyo] (part 6)], Dôbutsugaku Zasshi [Zoological Magazine, Tokyo], 3: 239-244 (in Japanese).

Ikeda, S. (1891e), "Rika daigaku hyôhon nihon tôsokurui mokuroku (dai nana kai)" [List of specimens of Japanese Cephalopoda at 
the Science College [Tokyo] (part 7)]. Dôbutsugaku Zasshi [Zoological Magazine, Tokyo], 3: 292-293 (in Japanese).

Ikeda, S. (1891f), "Rika daigaku hyôhon nihon tôsokurui mokuroku (dai hachi kai)" [List of specimens of Japanese Cephalopoda at the Science College [Tokyo] ([final] part 8)], Dôbutsugaku Zasshi [Zoological Magazine, Tokyo], 3: 324-331 (in Japanese).

International Commission on Zoological Nomenclature (1954), "Opinion 233, Suppression, under the plenary powers, of the name 'Octopodia' Schneider, 1784 (Class Cephalopoda), and of certain reputed names published by the same author in 1784," Opinions and Declarations of the International Commission on Zoological Nomenclature, 4 (23): 275-296.

International Commission on Zoological Nomenclature (1999), International Code of Zoological Nomenclature, 4th ed., International Trust for Zoological Nomenclature, London, p. 306.

Ishikawa, C., and Wakiya, Y. (1914), "On a new species of Moroteuthis from the Bay of Sagami, M. lönnbergii," Journal of the College of Agriculture, Imperial University, Tokyo, 4: 445-460, Pl. 45-46.

Isono, N. (1988), "Misaki rinkai jikkenjo o kyorai shita hitotachi: nihon ni okeru dôbutsugaku no tanjô" [The Visitors to Misaki Marine Research Station over the Years: The Birth of Zoology in Japan], Gakkai Shuppan Center, Tokyo.

Issel, R. (1908), "Diagnosi preliminari di un nuovo genre e di due nuove specie di cefalopodi appartenenti alla famiglia Cranchiidae raccolti della R. Nave 'Liguria'," Monitore Zoologico Italiano, 19: 102-104.

Keferstein, W. (1866), "Malacozoa,” Bronn's Klassen und ordnungen des Thierreiches, 157.

Khromov, D. N., Lu, C. C., Guerra, A., Dong, Zh., and Boletzky, S. v. (1998), “A synopsis of Sepiidae outside Australian waters,” Smithsonian Contributions to Zoology, 586: 77-157.

Kubodera, T., Piatkowski, U., Okutani, T., and Clarke, M. R. (1998), "Taxonomy and geography of the Family Onychoteuthidae (Cephalopoda: Oegopsida)," Smithsonian Contributions to Zoology, 586 (II): 277-291.

Leach, W. E. (1817), "Synopsis of the orders, families and genera of the class Cephalopoda," Zoological Miscellany, Vol. 3, Leach, W. E., and Nodder, R. P., Ed., London, pp. 137-141.

Lesson, R. P. (1830), "Mollusques, annélides et vers. 1. Mollusques," in Histoire naturelle des mollusques, annélides et vers recueillis dans le voyage autour du monde de la corvette de sa majesté, la 'Coquille, exécuté pendant les années 1822, 23, 24 et 1825, sous le commandement du Capitaine Duperrey, Zoologie, Vol. 2, No. 1, A. Bertrand, Paris, pp. 25-471, Pl. 10-16.

Lesueur, C. A. (1821), "Descriptions of several new species of cuttle-fish," Journal of the Academy of Natural Sciences of Philadelphia, 2: 86-101.

Lightfoot, J. (1786), A catalogue of the Portland Museum, London, p. 194.

Linnaeus, C. (1758), "Regnum animale, Mollusca" in Systema naturae, 10th ed., Vol. 1.

Lu, C. C. (1998), “A synopsis of Sepiidae in Australian waters (Cephalopoda: Sepioidea)," Smithsonian Contributions to Zoology, 586: $159-190$.

Massy, A. (1927), "The Cephalopoda of the South African Museum," Annals of the South African Museum, 25: 151-167.

Muus, B. (2002), "The Bathypolypus-Benthoctopus problem of the North Atlantic (Octopodidae, Cephalopoda)," Malacologia, 44: $175-222$.

Natsukari, Y. (1984), "Taxonomical and morphological studies on the loliginid squids-IV. Two new genera of the family Loliginidae," Venus, 43 (3): 229-239.

Norman, M. D. (1993), "Four new species of the Octopus macropus group (Cephalopoda: Octopodidae) from the Great Barrier Reef, Australia," Memoirs of the Museum of Victoria, 53 ('1992'): 267-308.

Norman, M. D., and Finn, J. (2001), "Revision of the Octopus horridus species-group, including erection of a new subgenus and description of two member species from the Great Barrier Reef, Australia," Invertebrate Taxonomy, 15: 13-35.

Norman, M. D., and Hochberg, F. G. (1994), "Shallow-water octopuses (Cephalopoda: Octopodidae) from Hong Kong's territorial waters," in The Malacofauna of Hong Kong and Southern China, Morton, B., Ed., Hong Kong University Press, Hong Kong, pp. 141-160.

Norman, M. D., Hochberg, F. G., and Lu, C. C. (1997), "Mollusca Cephalopoda: mid-depth octopuses (200-1000 m) of the Banda and Arafura Seas (Octopodidae and Alloposidae)," Mémoires du Musée National de l'Histoire Naturelle de Paris, 172: $357-383$.

Okada, Y. K. (1927a). "Contribution à l'étude des céphalopodes lumineux (notes préliminaires), IV: Ommastrephes volatilis Sasaki est une forme lumineuse; établissement d'un nouveau genre: Ornithoteuthis," Bullétin de l'Institute Océanographique de Monaco, 494: $1-7$.

Okada, Y. K. (1927b), "Céphalopodes japonais des collections du Muséum," Bullétin du Musée National de l'Histoire Naturelle de Paris, 1927: 93-98.

Okutani, T. (2002), "Raberu wa kataru—Tokyo daigaku sôgô kenkyû hakubutsukan no tôsokurui hyôhon chôsa yoroku" [The labels talk: additional comments on the survey of Cephalopoda specimens in Tokyo University Museum], Chiribotan, 33: 73-79.

Okutani, T., Tagawa, M., and Horikawa, H. (1987), Cephalopods from Continental Shelf and Slope around Japan, Japan Fisheries Resources Conservation Association, Tokyo, p. 194.

d'Orbigny, A. D. (1826), "Tableau méthodique de la classe des céphalopodes," Academie des Sciences Naturelles de Paris (Ser. 1), 7 (1): 96-169, 245-314 ('1825').

d'Orbigny, A. (1839-48), "Histoire naturelle générale et particulière des céphalopodes acétabulifères, Tome 1. Texte," in Histoire naturelle générale et particulière des céphalopodes acétabulifères vivants et fossiles, Férussac, A. E., and d'Orbigny, A., Ed., J.-B. Bailliere, Paris, pp. 1-366 ('1834-48').

Ortmann, A. (1888), "Japanische Cephalopoden,” Zoologische Jahrbucher Abt. Systematik, 3: 639-670, Pl. XX-XXV.

Rafinesque, C. S. (1814), "Classe Malacosia-les mollusques," in Précis des découvertes sémiologiques ou zoologiques et botaniques entre 1800 et 1814, Palermo.

de Rochebrune, A. T. (1884a), "Étude monographique de la famille des Sepiadae," Bullétin de la Societé Philomathique de Paris (Ser. 7), 8 74-122.

de Rochebrune, A. T. (1884b), "Étude monographique de la famille des Eledonidae," Bullétin de la Societé Philomathique de Paris (Ser. 7), 8: 152-163 ( fide Norman, 1993). 
Roper, C. F. E., and Hochberg, F. G. (1988), "Behavior and systematics of cephalopods from Lizard Island, Australia based on color and body patterns," Malacologia, 29 (1): 153-193.

Roper, C. F. E., and Sweeney, M. (1978), "A Catalogue of the type specimens of recent Cephalopoda in the National Museum of Natural History," Smithsonian Contributions to Zoology, 287: 1-18.

Rüppell, E. (1844), "Intorno ad alcuni cefalopodi del mare di Messina," Giornale del Gabinetto Letterario di Messina, 27-28: 129135 ( fide Roper and Sweeney, 1998).

Rüppell, E., and Leuckart, F. S. (1828). Atlas zu der reise im nordlichen Afrika von Eduard Rüppell, Neue wirbellose Thiere des rotten Meers, Frankfurt am Main.

Salcedo-Vargas, M. A. (1997), "Cephalopods from the Netherlands Indian Ocean Programme (NIOP)—II. Mastigoteuthid lineage and related forms," Beaufortia, 47 (3): 91-108.

Salcedo-Vargas, M. A., and Okutani, T. (1994), "New classification of the squid family Mastigoteuthidae (Cephalopoda: Oegopsida)," Venus, 53 (2): 119-127.

Sasaki, M. (1909), "Nihon ni san suru jukkyaku tôsokurui (tsudzuki)" [Native decapod cephalopods of Japan (continued)], Dôbutsugaku Zasshi [Zoological Magazine, Tokyo], 21 (253): 481-486, Pl. 10 (Figs. 1-16) [following p. 498] (in Japanese).

Sasaki, M. (1910). "Nihon ni san suru jukkyaku tôsokurui (tsudzuki)" [Native decapod cephalopods of Japan (continued)], Dôbutsugaku Zasshi, 22 (261): 363-371, Pl. 12 (Figs. 1-13, 14A, B) [following p. 406], Pl. 13 (Figs. 1-12) [following p. 436] (in Japanese).

Sasaki, M. (1913a), "Nihon ni san suru jukkyaku tôsokurui (tsudzuki)" [Native decapod cephalopods of Japan (continued)], Dôbutsugaku Zasshi, 25 (292): 73-86, 11 textfigs., Pl. 2 figs. 1-16, Pl. 3 figs. 1-5, 6A, B (in Japanese).

Sasaki, M. (1913b), "Nihon ni san suru jukkyaku tôsokurui (tsudzuki)" [Decapod cephalopods native to Japan (continued)] (Sepiolidae), Dôbutsugaku Zasshi, 25: 247-252 (in Japanese).

Sasaki, M. (1913c), "Nihon ni san suru jukkyaku tôsokurui (Sepiolidae) (tsudzuki, kan)" [Decapod cephalopods native to Japan (Sepiolidae) (continued to completion)], Dôbutsugaku Zasshi, 25: 397-403 (in Japanese).

Sasaki, M. (1914a), "Notes on the Japanese Myopsida," Nihon Dôbutsugaku Iho [Annotationes Zoologicae Japonenses], 8: 587629, Pl. XI (Figs. 1-12), XII (Figs. 1-6), 1 textfig.

Sasaki, M. (1914b), "Observations on hotaru-ika Watasenia scintillans," Journal of the College of Agriculture, Tohoku Imperial University, Sapporo, 6: 75-107, Pl. 1-3, 1 textfig.

Sasaki, M. (1915), "On three interesting new oegopsids from the Bay of Sagami," Journal of the College of Agriculture, Tohoku Imperial University, Sapporo, 6: 131-150, Pl. IV (Figs. 1-13); 4 textfigs.

Sasaki, M. (1916), "Notes on oegopsid cephalopods found in Japan," Nihon Dôbutsugaku Iho [Annotationes Zoologicae Japonenses], 9 (2): 89-120, Pl. III (Figs. 1-5).

Sasaki, M. (1917a), "Notes on the Cephalopoda. I. On the male of Amphitretus pelagicus Hoyle,” Nihon Dôbutsugaku Iho, 9: 361364.

Sasaki, M. (1917b), "Notes on the Cephalopoda. II. Diagnoses of four new species of Polypus," Nihon Dôbutsugaku Iho, 9: $364-367$.

Sasaki, M. (1920), "Report on cephalopods collected during 1906 by the United States Bureau of Fisheries steamer 'Albatross' in the northwestern Pacific," Proceedings of the United States National Museum, 57: 163-203.

Sasaki, M. (1921), "On the life history of an economic cuttlefish of Japan, Ommastrephes sloani pacificus," Transactions of the Wagner Free Institute of Science of Philadelphia, 9 (2): 1-25.

Sasaki, M. (1923), "On an adhering habit of a pygmy cuttlefish, Idiosepius pygmaeus Steenstrup," Nihon Dôbutsugaku Iho [Annotationes Zoologicae Japonenses], 10 (6): 209-213.

Sasaki, M. (1925), "Surume ika Ommastrephes sloani pacificus (Steenstrup) no kaibô" [Anatomy of the surume squid, Ommastrephes sloani pacificus (Steenstrup)], Dôbutsugaku Zasshi [Zoological Magazine, Tokyo], 37: 240-261, textfigs. 1-7 (in Japanese).

Sasaki, M. (1929), "A Monograph of the dibranchiate cephalopods of the Japanese and adjacent waters," Journal of the College of Agriculture, Tohoku Imperial University, Sapporo, 20 (suppl. 10): 1-357, Pl. I-XXX, 179 textfigs.

Sasaki, M. (1929a), "Supplementary notes on the life history of an economic cuttlefish, Ommastrephes sloani pacificus Steenstrup," Japanese Journal of Zoology, Tokyo, 2: 199-211.

Steenstrup, J. (1856), "Hectocotyldannelsen hos Octopodslaegterne Argonauta og Tremoctopus, oplyst ved, Iagttagelse af lignende Dannelser hos Bläksprutterne i Almindelighed.” Kongelige Danske Videnskabernes Selskabs Skrifter. (Series 5), 4: 185-216.

Steenstrup, J. (1859), "Prof. Stp. gav en meddelse om to nye cephalopodslægter Bolitaena og Haliphron," Videnskabelige Meddelelser fra den Naturhistoriske Forening i Kjøbenhavn, 1858: 180-183.

Steenstrup, J. (1861), "Forevisning af en afdeling af Museets seneste Forøgelser af Blæksprutteklassen med bemærkninger om de enkelte arter," Videnskabelige Meddelelser fra den Naturhistoriske Forening i Kjøbenhavn, 1860-1861: 332-333.

Steenstrup, J. (1875), "Hemisepius, en ny Släegt af Sepia-Blaeksprutternes Familie, med Bemaekninger om Sepia-Formerne i Almindelighed." Kongelige Danske Videnskabernes Selskabs Skrifter (Series 5), 10 (7): 465-482.

Steenstrup, J. (1880), "Orientering i de Ommastrephagtige Blæksprutters indbyrdes forhold," Oversift over det Kongelige Danske Videnskabernes Selskabs Forhandlinger, 1880-1881: 73-110.

Steenstrup, J. (1881), "Sepiadarium og Idiosepius to nye slägter af sepiernes familie. Med Bemaerknin ger om de to beslaegted e former Sepioloidea d'Orb. og Spirula Lmk. Det," Kongelige dansk Videnskabernes Selskabs Skrifter. (Series 6), 1: $213-242$.

Sweeney, M. J., and Roper, C. F. E. (1998), "Classification, type localities, and type repositories of recent Cephalopoda," Smithsonian Contributions to Zoology, 586: 561-599.

Sweeney, M. J., and Vecchione, M. (1998), "Generic and specific names introduced in the squid Family Loliginidae (Cephalopoda: Myopsida)," Smithsonian Contributions to Zoology, 586: 223-237.

Taki, I. (1942), “The Mollusca of the Inland Sea of Japan (1)," Venus [Japanese Journal of Malacology], 12: 64-80.

Taki, I. (1962), "On species newly added to the fauna of Japanese Cephalopoda," Dôbutsugaku Zasshi [Zoological Magazine, 
Tokyo], 71 (11-12): 397-398.

Taki, I. (1963), "On four newly known species of Octopoda from Japan,” Journal of the Faculty of Fisheries and Animal Husbandry, Hiroshima University, 5: 57-83, Pl. 1-5.

Taki, I. (1964), "On eleven new species of Cephalopoda from Japan, including two new species of Octopodinae," Journal of the Faculty of Fisheries and Animal Husbandry, Hiroshima University, 5: 277-330, Pl. 1-7.

Taki, I., and Igarashi, T. (1967), "A list of cephalopod specimens in the Fisheries Museum, Faculty of Fisheries, Hokkaido University. (Sasaki collection and specimens collected by the Marine Zoological Laboratory)," Fisheries Museum, Faculty of Fisheries, Hokkaido University, Contribution, 7: 1-26 (in Japanese).

Thore, S. (1949), "Investigations of "Dana" Octopoda. Part I. Bolitaenidae, Amphitretidae, Vitreledonellidae and Alloposidae," Dana Report, 33: 1-75, 69 figs.

Toll, R. B., and Voss, G. L. (1998), "The systematic and nomenclatural status of the Octopodinae described from the West Pacific region," Smithsonian Contributions to Zoology, 586: 489-520.

Troschel, H. (1857), "Bemerkungen über die Cephalopoden von Messina," Archiv für Naturgeschichte, 23 (Jahrg. 1): 40-76, Plate 4.

Tsuchiya, K., and Okutani, T. (1991), "Growth stages of Moroteuthis robusta (Verrill, 1881) with the re-evaluation of the genus," Bulletin of Marine Science, 49 (1-2): 137-147.

Vecchione, M., Collins, M. A., and Sweeney, M. J. (2002), "Systematics, ecology and biology of cirrate octopods: workshop report," Bulletin of Marine Science, 71: 79-96.

Voss, N. A. (1969). "A monograph of the Cephalopoda of the North Atlantic. The Family Histioteuthidae," Bulletin of Marine Science, 19: 713-867.

Voss, N. A. (1985), "Systematics, biology and biogeography of the cranchiid cephalopod genus Teuthowenia (Oegopsida)," Bulletin of Marine Science, 36: 1-85.

Voss, N. A., Nesis, K. N., and Rodhouse, P. G. (1998), "The cephalopod family Histioteuthidae (Oegopsida): systematics, biology, and biogeography," Smithsonian Contributions to Zoology, 586: 293-372.

Wakiya, Y., and Ishikawa, M. (1921), "Hôsan jukkyaku tôsokurui heiganrui ni tsuite (yon shinshu)" [On the nationally occurring decapod Cephalopoda, Myopsida (four new species)], Dôbutsugaku Zasshi [Zoological Magazine, Tokyo], 33: 279-292, Pl. 1, 2 (in Japanese).

Wormuth, J. H. (1998), "Workshop deliberations on the Ommastrephidae: a brief history of their systematics and a review of the systematics, distribution, and biology of the genera Martialia Rochebrune and Mabille, 1889, Todaropsis Girard, 1890, Dosidicus Steenstrup, 1857, Hyaloteuthis Gray, 1849, and Eucleoteuthis Berry, 1916," Smithsonian Contributions to Zoology, 586: 373 -383.

Wülker, G. (1910), “Über Japanische Cephalopoden. Beiträge zur Kenntnis der Systematik und Anatomie der Dibranchiaten. Beiträge zur Naturgeschichte Ostasiens," Abhandlungen der Mathematisch-Physikalischen Classe der Königlich Bayerischen Akademie der Wissenchaften 3 (suppl. 1, Doflein F., Ed.), pp. 1-77, figs. 1-58. Munchen. 


\section{Appendix: Annotated Summary of the Cephalopoda Specimens in the Zoological Institute of Tokyo Imperial University Listed by Ikeda $(1890 a, b, 1891 a-f)$.}

Lot numbers marked with an asterisk (*) have been matched (positively or tentatively) with specimens in the present collection (see above). Occasional spelling errors of taxa in Ikeda's original texts have been ignored, otherwise the identifications are those stated by Ikeda (for updated identifications, refer to main catalogue listed above). The only measurements recorded here are total length and/or mantle length where stated by Ikeda. In several instances Ikeda also includes a number of other measurements (including arm, tentacle, fin lengths and widths). Note that Ikeda stated all measurements in $\mathrm{cm}$ (retained here: $c f$. usual convention of using $\mathrm{mm}$ ). Numbers prefixed ' $\mathrm{M}$ ' in parentheses are the original Meiji reign years used throughout Ikeda's papers; numbers without a prefix in the 'Specimens' column are mantle lengths in $\mathrm{cm}$. Other abbreviations: ALO, arm length order; ML, mantle length (in $\mathrm{cm}$ ); Pref., Prefecture; Prov., Province; TL, total length. Comments are brief extracts from Ikeda's sometimes extensive notes on each lot.

\section{Family Octopodidae.}

1. Octopus octopodia, 'madako' [common octopus] [cf. Octopus vulgaris Cuvier sensu Sasaki, 1929] (Ikeda, 1890a: 481).

\begin{tabular}{|c|c|c|c|c|}
\hline lot\# & Specimens & Date and Collectors & Locality & Comments \\
\hline 1 & 1 spec. & Nov., 1882 (M 15). & Tokyo market & $\begin{array}{l}\text { TL } 40 \mathrm{~cm} \text {. Large, but hectocotylized } \\
\text { arm missing. }\end{array}$ \\
\hline $2^{*}$ & 1 spec. & $\begin{array}{l}\text { Summer, } 1882 \\
\text { (M 15), Mitsukuri. }\end{array}$ & Bingo [Hiroshima Pref.], Tomonotsu. & TL $10 \mathrm{~cm}$. 'Octopus kagoshimensis.' \\
\hline $3 *$ & 2 specs. & $\begin{array}{l}\text { April, } 1884 \text { (M 17), Mitsukuri, } \\
\text { Ishikawa and Okada. }\end{array}$ & $\begin{array}{l}\text { [Suruga Bay], Shunshu [Shizuoka Pref.] } \\
\text { Enoura [nr. Numazu]. }\end{array}$ & TL $10 \mathrm{~cm}, 7 \mathrm{~cm}$ \\
\hline 4 & 2 specs. & Dec., 1888 (M 21). & $\begin{array}{l}\text { Soushu [Sagami Prov.], [Kanagawa } \\
\text { Pref.] Miura, Misaki. }\end{array}$ & $\begin{array}{l}\mathrm{TL} \quad 7 \mathrm{~cm}, \quad 6 \mathrm{~cm} \text {. Compared with } \\
\text { O. globosus Appellöf and O. bermu- } \\
\text { densis Hoyle. }\end{array}$ \\
\hline 5 & 5 specs. & $\begin{array}{l}\text { April, } 1884 \text { (M 17), Mitsukuri, } \\
\text { Ishikawa and Okada. }\end{array}$ & $\begin{array}{l}\text { [Suruga Bay], Shunshu } \quad \text { [Shizuoka } \\
\text { Pref.], Enoura. }\end{array}$ & TL $2.5 \mathrm{~cm}$ \\
\hline $6^{*}$ & 1 spec. & July, 1885 (M 18), Okada. & Echizen [Fukui Pref.], Sakai harbour. & TL $12 \mathrm{~cm}$. \\
\hline 7 & 3 specs. & Sept., 1886 (M 19). & Tokyo market & TL $40 \mathrm{~cm}$ \\
\hline $8^{*}$ & 2 specs. & $\begin{array}{l}\text { April, } 1885 \text { (M 18), Ishikawa, } \\
\text { Okada. }\end{array}$ & Boshu [Chiba Pref.], Kominato. & TL $8 \mathrm{~cm}, 2.5 \mathrm{~cm}$ \\
\hline 9 & 1 spec. & Aug., 1884 (M 17), Imai. & $\begin{array}{l}\text { Sagami [Kanagawa Pref.], } \\
\text { off Kannon-zaki [Kannon Point]. }\end{array}$ & $\begin{array}{l}\text { Specimen with branched arms } \\
\text { (total of } 78 \text { arm tips). }\end{array}$ \\
\hline
\end{tabular}

2. Octopus megalocyathus Couthoy in Gould sensu Ikeda. 'yanagidako' [willow octopus] (Ikeda 1890a: 482)

[cf. Octopus conispadiceus (Sasaki, 1917)].

\begin{tabular}{|c|c|c|c|c|}
\hline lot\# & Specimens & Date & Locality & Comments \\
\hline 1 & & 32 (M 15). & va Pref.], Miura, Misaki. & TL $1 \mathrm{~m}$, arms 1 and $2>80 \mathrm{~cm}$. ALO $2=1.3 .4$ \\
\hline
\end{tabular}

3. Octopus macropus Risso sensu Ikeda. ‘shakushidako' [ladle-ligula octopus], 'ashinaga ihidako' [long-armed, boiled-rice octopus] (Ikeda, 1890b: 528-529) [cf. 'Octopus' minor (Sasaki, 1920)].

\begin{tabular}{|c|c|c|c|c|}
\hline lot\# & Specimens & Date and Collectors & Locality & Comments \\
\hline $1 *$ & 7 specs. & 1.vii.1882 (M 15). & Tokyo Bay, Haneda. & TL $40-60 \mathrm{~cm}$. \\
\hline $2 *$ & 3 specs. & 7.xii.1882 (M 15). & Tokyo market. & TL 22-55 cm. cf. O. pusillus Gould \\
\hline 3 & 2 specs. & (no data) & Naguchi (hiragana script). & $\begin{array}{l}\text { TL } 25 \mathrm{~cm} . c f \text {. Tryon, 1879: 112, Pl. } 31 \text { figs. } 32 \text {, } \\
33 .\end{array}$ \\
\hline $4^{*}$ & 1 spec. & April, 1885 (M 18). & Boshu [Chiba Pref.], Kominato. & TL $32 \mathrm{~cm} . c f$. O. januarius Steenstrup in Hoyle. \\
\hline $5^{*}$ & 2 specs. & $\begin{array}{l}\text { Summer, } 1882 \text { (M 15), } \\
\text { Mitsukuri and Kikuchi et al. }\end{array}$ & $\begin{array}{l}\text { Bitchu [Okayama Pref.] } \\
\text { Yukashima. }\end{array}$ & Both TL $22 \mathrm{~cm}$ \\
\hline 6 & 1 spec. & (no data) & [Kanagawa Pref.], Enoshima. & $\begin{array}{l}\text { TL } 28 \mathrm{~cm} \text {. Resembles Octopus pusillus: Tryon } \\
\text { or O. globosus: Appellöf. }\end{array}$ \\
\hline 7 & 2 specs. & (no data) & (no data) & $\begin{array}{l}\mathrm{TL}<6 \mathrm{~cm} . c f . \text { O. macropus or } O \text {. bermudensis } \\
\text { Hoyle. }\end{array}$ \\
\hline
\end{tabular}

Discussion of this species includes (with text excerpts): Octopus macropus Risso, O. cuvieri d'Orbigny, O. januarii Steenstrup, O. pusillus Gould and O. kagoshimensis Ortmann. The specimen in lot \#4 is the holotype of 'Octopus' pardalis (Sasaki, 1929).

4. Octopus ocellatus Gray, 1849. 'ihidako' ['iidako', boiled-rice octopus] (Ikeda, 1891a: 25) [Amphioctopus fangsiao (d'Orbigny, 1841)].

\begin{tabular}{|c|c|c|c|c|}
\hline lot\# & Specimens & Date and Collector & Locality & Comments \\
\hline $1 *$ & 2 males, 2 females. & 7.xii.1882 (M 15). & Tokyo market. & $\begin{array}{l}\text { TL } 18,15 \mathrm{~cm} \text { (males), } 13.5,7 \mathrm{~cm} \\
\text { (females). }\end{array}$ \\
\hline 2 & 1 female. & $\begin{array}{l}\text { Aug., } 1884 \text { (M 17), } \\
\text { Okada. }\end{array}$ & $\begin{array}{l}\text { [Japan Sea], Tango [Kyoto Pref.], } \\
\text { Miyazu. }\end{array}$ & $\mathrm{TL} 18 \mathrm{~cm}$ \\
\hline 3 & 1 female. & Aug., 1891 (M 24). & [Kanagawa Pref.], Misaki. & TL $14.5 \mathrm{~cm}$ \\
\hline 4 & 1 spec. & 7.xii.1882 (M 15). & Nabechi (hiragana script). & TL $56 \mathrm{~cm}$ \\
\hline
\end{tabular}




\section{Family Tremoctopodidae.}

Parasira catenulata 'Blainville' [Octopus catenulatus Philippi?] sensu Ikeda (1891a: 26). [Ocythoe tuberculata Rafinesque, 1814].

\begin{tabular}{|c|c|c|c|c|}
\hline lot\# & Specimens & Date and Collector & Locality & Comments \\
\hline $1 *$ & 1 female. & (no data) & Boshu [Chiba Pref.], Fuku-ura. & $\begin{array}{l}\text { TL } 30 \mathrm{~cm} \text {, arm \#1 } 20 \mathrm{~cm} \text {, body } 8 \mathrm{~cm} \text { long, } \\
6 \mathrm{~cm} \text { wide. }\end{array}$ \\
\hline $2 *$ & 1 female. & $\begin{array}{l}\text { Sept., } 1885 \text { (M 18), } \\
\text { Sakamoto }\end{array}$ & Boshu, Kashiwazaki. & TL $24.5 \mathrm{~cm}$ \\
\hline
\end{tabular}

Ikeda includes as a synonym "P. pictus Brock".

\section{Family Argonautidae.}

1. Argonauta hians Lightfoot, 1786, sensu Ikeda. 'takobune' [octopus boat] (Ikeda, 1891a: 27).

\begin{tabular}{ccccc}
\hline lot\# & Specimens & Date and Collector(s) & Locality & Comments \\
\hline 1 & 3 shells & (no data) & (no data) & height $9.5 \mathrm{~cm}, 7 \mathrm{~cm}, 6.3 \mathrm{~cm}$ \\
2 & 1 spec. & (no data) & (no data) & height $5.5 \mathrm{~cm}$ \\
3 & 1 shell & (no data) & (no data) & height $6.5 \mathrm{~cm}$ \\
\hline
\end{tabular}

2. Argonauta argo Linnaeus, 1758, sensu Ikeda. 'takobune' (Ikeda, 1891a: 27).

\begin{tabular}{ccccc}
\hline lot\# & Specimens & Date and Collector(s) & Locality & Comments \\
\hline 1 & 1 spec. & (no data) & (no data) & shell height $15 \mathrm{~cm}$ \\
\hline
\end{tabular}

3. Argonauta fragilis Parkinson, 1856, sensu Ikeda (1891a: 27) [cf. A. argo].

\begin{tabular}{ccccc}
\hline lot\# & Specimens & Date and Collector(s) & Locality & Comments \\
\hline 1 & 2 shells & (no data) & (no data) & $3.3 \mathrm{~cm}, 1.8 \mathrm{~cm}$ \\
\hline
\end{tabular}

4. Argonauta owenii Adams and Reeve, 1850, sensu Ikeda (1891a: 28) [cf. A. hians].

\begin{tabular}{|c|c|c|c|c|}
\hline lot\# & Specimens & Date and Collector & Locality & Comments \\
\hline 1 & 4 shells & April, 1887 (M 20), Okada. & $\begin{array}{l}\text { Izu Shichito [Izu Shoto Islands], } \\
\text { Niijima. }\end{array}$ & $\begin{array}{l}3 \text { specs. height } 5 \mathrm{~cm}, 1 \mathrm{spec} \text {. height } \\
3 \mathrm{~cm} .\end{array}$ \\
\hline
\end{tabular}

\section{Family Loliginidae.}

1. Loligo sumatrensis d'Orbigny, 1848, sensu Ikeda. 'hi-ika' (Ikeda, 1891a: 28).

\begin{tabular}{ccccc}
\hline lot\# & Specimens & Date & Locality & Comments \\
\hline $1 *$ & 3 males, 4 females. & 1.xii.1885 (M 18). & Tokyo market & TL 12-14 cm. \\
\hline
\end{tabular}

cf. Loliolus japonicus 996.5.109.

2. Loligo edulis Hoyle, 1885. 'yari-ika' [spear squid] (Ikeda, 1891a: 29) [Uroteuthis edulis].

\begin{tabular}{ccccc}
\hline lot\# & Specimens & Date and Collector & \multicolumn{1}{c}{ Locality } & \\
\hline $1^{*}$ & 1 female. & April, 1887 (M 20), Okada. & Izu [Shoto Islands group], Oshima. & TL 32 cm, ML 16 cm. \\
2 & 1 female. & (no data) & Soshu [Kanagawa Pref.], Enoshima. & TL 20 cm. \\
\hline
\end{tabular}

*cf. Uroteuthis kensaki 996.5.117.

3. Loligo bleekeri Keferstein, 1866. 'yari-ika' (Ikeda, 1891a: 30 and 1891b: 61; 1891f: 331థ).

\begin{tabular}{|c|c|c|c|c|}
\hline lot\# & Specimens & Date & Locality & Comments \\
\hline 1 & 1 male. & 7.xii.1882 (M 15). & Tokyo market. & TL $54 \mathrm{~cm}$, ML $24(34) \uparrow \mathrm{cm}$. \\
\hline 2 & 1 male, 1 female. & 1st Feb. (year unknown). & Tokyo market. & TL $22,23 \mathrm{~cm}$ \\
\hline 3 & 1 male, 2 females. & 20.xii.1885 (M 18). & Tokyo market. & ML $23 \mathrm{~cm}$ \\
\hline
\end{tabular}

- see "Corrections and omissions," below. 


\section{Genus Sepioteuthis.}

Sepioteuthis lessoniana Lesson, 1830 (Ikeda, 1891b: 61-62).

\begin{tabular}{|c|c|c|c|c|}
\hline lot\# & Specimens & Date and Collector & Locality & Comments \\
\hline $1^{*}$ & 1 male (37). & (no data) & $\begin{array}{l}\text { [Japan Sea], Etchu [Toyama Pref.], } \\
\text { Namerikawa. }\end{array}$ & TL $91 \mathrm{~cm}$. \\
\hline 2 & 1 male. & (no data) & Tokyo Bay, Haneda. & TL $75 \mathrm{~cm}$. \\
\hline 3 & 1 female. & (no data) & Tokyo market. & TL $28 \mathrm{~cm}$. \\
\hline $4 *$ & 4 males, 1 female. & Sept., 1885 (M 18). & Tokyo market. & TL $26-29 \mathrm{~cm}$. \\
\hline 5 & 1 female. & (no data) & (no data) & TL $42 \mathrm{~cm}$. \\
\hline $6^{*}$ & 1 male. & $\begin{array}{l}\text { April, } 1887 \text { (M 20), } \\
\text { Okada. }\end{array}$ & $\begin{array}{l}\text { [Sagami Bay], Izu Shichito [Izu Shoto } \\
\text { Islands], Oshima, Habuminato. }\end{array}$ & TL $23 \mathrm{~cm}$. \\
\hline 7 & 1 spec. (8). & (no data) & Tokyo market. & - \\
\hline
\end{tabular}

\section{Family Sepiolidae.}

Inioteuthis morsei Verrill, 1881, sensu Ikeda (Ikeda, 1891b: 62-63). [cf. Euprymna morsei, and E. berryi Sasaki, 1929].

\begin{tabular}{|c|c|c|c|c|}
\hline lot\# & Specimens & Date and Collectors & Locality & Comments \\
\hline 1 & 2 males, 4 females. & 1.x.1885 (M 18). & Tokyo market. & TL $5.5-9.0 \mathrm{~cm}$ \\
\hline 2 & 1 ?female. & $\begin{array}{l}\text { April, } 1884 \text { (M 17), Mitsukuri } \\
\text { and Ishikawa. }\end{array}$ & $\begin{array}{l}\text { Shunshu [Shizuoka Pref.], } \\
\text { Enoura. }\end{array}$ & TL $2.5 \mathrm{~cm}$ \\
\hline $3^{*}$ & 1 male, 1 female. & (no date), coll. Okada. & $\begin{array}{l}\text { [Osaka Bay], Awaji [Island], } \\
\text { Tsume-gun, Shumoto. }\end{array}$ & TL $6 \mathrm{~cm}$ \\
\hline 4 & 1 female, 1 juv. & (no data) & $\begin{array}{l}\text { Soshu [Kanagawa Pref.], } \\
\text { Miura-gun, Misaki. }\end{array}$ & $\mathrm{TL} 4.5 \mathrm{~cm},<2.0 \mathrm{~cm}$ \\
\hline 5 & 2 females. & (no data) & (no data) & TL c. $4 \mathrm{~cm}$. \\
\hline
\end{tabular}

Includes Sepiola bursa Pfeffer as a synonym.

\section{Family Chiroteuthidae.}

Chiroteuthis veranyi Ferussac, 1835, sensu Ikeda (1891c: 144).

\begin{tabular}{cccc}
\hline lot\# & Specimens & \multicolumn{1}{c}{ Date } & \multicolumn{1}{c}{ Locality } \\
\hline $1 *$ & 2 specs. (14) & Sept., 1887 (M 20). & Soshu [Kanagawa Pref.], \\
& & Miura-gun, Misaki, [Tokyo] & Imp. Univ. Res. Stn. \\
\hline
\end{tabular}

\section{Family Ommastrephidae.}

1. Ommastrephes pacificus Steenstrup sensu Appellöf. 'surume-ika' [Todarodes pacificus (Steenstrup, 1880)] (Ikeda, 1891c: 145).

\begin{tabular}{ccccc}
\hline lot\# & Specimens & Date & Locality & Comments \\
\hline 1 & 2 specs. & Sept., $1882($ M 15). & Tokyo market & ML 20.5 cm (both). \\
\hline
\end{tabular}

2. Ommastrephes, sp. 'surume-ika' (Ikeda, 1891c: 146).

\begin{tabular}{ccccc}
\hline lot\# & Specimens & Date & Locality & Comments \\
\hline 1 & 7 specs., incl. 2 males (21.5). & (no data) & (no data) & All similar size. \\
\hline
\end{tabular}

3. Ommastrephes insignis Gould sensu Ikeda. 'surume-ika' (Ikeda, 1891c: 146).

\begin{tabular}{|c|c|c|c|c|}
\hline lot\# & Specimens & Date & Locality & Comments \\
\hline $1 *$ & 1 female, 1 male (24). & Sept., 1885 (M 18). & Soshu [Kanagawa Pref.], Odawara. & $\begin{array}{l}\text { Both: fin } 10 \mathrm{~cm} \text {, tentacles } 38 \mathrm{~cm} \text {, } \\
\text { TL } 68 \mathrm{~cm} .\end{array}$ \\
\hline
\end{tabular}

cf. Ommastrephes bartramii 996.5 .53 (coll. Nov., 1885).

4. Ommastrephes, sp. 'surume-ika' (Ikeda, 1891c: 147).

\begin{tabular}{llcll}
\hline lot\# & \multicolumn{1}{c}{ Specimens } & Date & Locality & \\
\hline 1 & 4 specs. $(14)$ & (no data) & Tokyo market. & Fin $6 \mathrm{~cm}$, width $8.5 \mathrm{~cm}$, tentacles $24 \mathrm{~cm}$. \\
$2 *$ & 5 specs. & Sept., $1885(\mathrm{M} 18)$. & Tokyo market. & Immature? \\
\hline
\end{tabular}


Histioteuthis sp. 'kuragedako' [jellyfish octopus] (Ikeda, 1891d: 240).

\begin{tabular}{ccccc}
\hline lot\# & Specimens & Date and Collector & Locality & Comments \\
\hline 1 & 1 spec. (18). & 4.iv.1889 (M 22), Fukuji Sakamoto. & Boshu [Chiba Pref.], Yoshihama. & TL 43 cm. \\
\hline
\end{tabular}

pp. 240-241 specimen discussed in some detail.

Onychoteuthis sp. 'beni-ika' [red squid] (Ikeda, 1891d: 241) [Watasenia scintillans (Berry, 1911)].

\begin{tabular}{ccclc}
\hline lot\# & Specimens & \multicolumn{1}{c}{ Date and Collector } & \multicolumn{2}{c}{ Locality } \\
\hline $1^{*}$ & 25 specs. (4) & 16.ii.1891 (M 24), Fukuji Sakamoto. & Boshu [Chiba Pref.], Nishizaki & TL 3.5 cm. Sasaki, 1914b lot \#3; \\
& & & [Nishimisaki]. & 1916 lot \#1. \\
\hline
\end{tabular}

pp. 241-242 discusses these specimens in some detail. Ikeda states that 10 specimens were sent to England [BMNH?]. This lot now contains only 16 specimens (see main ZUMT catalogue under W. scintillans). The other nine or ten have not been located at BMNH (A. MacLellan, pers. comm.).

\section{Family Sepiidae, Genus Sepia}

1. Sepia esculenta Hoyle, 1885. 'goto-ika' [Goto's cuttlefish?] (Ikeda, 1891d: 242).

\begin{tabular}{clllc}
\hline lot\# & \multicolumn{1}{c}{ Specimens } & \multicolumn{1}{c}{ Date } & \multicolumn{1}{c}{ Locality } & \multicolumn{1}{c}{ Comments } \\
\hline 1 & 2 males $(21,17)$, & Sept., 1881 (M 14), and Sept., & Tokyo market & $c f$. Sasaki, 1914a: 611, lot \#1. \\
& 2 females $(22,13)$. & 1885 (M 18). & & \\
\hline
\end{tabular}

2. Sepia sp. 'hari-ika' [needle cuttlefish] (Ikeda, 1891d: 243).

\begin{tabular}{cclc}
\hline lot\# & \multicolumn{1}{c}{ Specimens } & \multicolumn{1}{c}{ Date } & \multicolumn{1}{c}{ Locality } \\
\hline 1 & 24 specs. (8-12). & Sept., 1881 (M 14), and Sept., & Tokyo market \\
& & & \\
& & & \\
\hline
\end{tabular}

2 (sic). Sepia peterseni Appellöf, 1886. 'kaminari-ika' [thunder squid] (Ikeda, 1891e: 292).

\begin{tabular}{|c|c|c|c|c|}
\hline lot\# & Specimens & Date and Collector & Locality & Comments \\
\hline 1 & 12 specs. (both sexes). & Oct., 1885 (M 18). & Soshu [Kanagawa Pref.], Odawara. & Largest ML $10 \mathrm{~cm}$. \\
\hline 2 & 11 specs. (both sexes). & Oct., 1885 (M 18), Fukuji Sakamoto. & Soshu, Odawara. & ML $8.5-11.0 \mathrm{~cm}$ \\
\hline
\end{tabular}

3. Sepia kobiensis Hoyle, 1885 (Ikeda, 1891f: 324).

\begin{tabular}{cccc}
\hline lot\# & Specimens & Date and Collectors & Locality \\
\hline 1 & 2 females (6). & April, 1884 (M 17), Mitsukuri and Ishikawa. & Shunshu [Shizuoka Pref.], Enoura. \\
\hline
\end{tabular}

4. Sepia andreanoides Hoyle, 1885. 'suji-ika' (Ikeda, 1891f: 325).

\begin{tabular}{ccccc}
\hline lot\# & Specimens & Date & Locality & Comments \\
\hline 1 & 4 males, 2 females. & Sept., 1885 (M 18). & Tokyo market. & Largest ML 9 cm. \\
\hline
\end{tabular}

5. Sepia sp. 'suji-ika,' 'sa-ika' (Ikeda, 1891f: 326-328).

\begin{tabular}{|c|c|c|c|c|}
\hline lot\# & Specimens & Date and Collector & Locality & Comments \\
\hline 1 & 1 male (18). & 22.x.1885 (M 18). & [Kanagawa Pref.], Enoshima. & - \\
\hline 2 & $\begin{array}{l}2 \text { males }(14.5) \\
1 \text { female }(12.5)\end{array}$ & 20.ii.1889 (M 22), Fukuji Sakamoto. & Boshu [Chiba Pref.], Kachiyama [Katsuyama]. & - \\
\hline 3 & 1 male (18.5). & 15.x.1883 (M 16). & $\begin{array}{l}\text { Boshu [Kanagawa Pref., Yokohama], Fuku- } \\
\text { ura. }\end{array}$ & - \\
\hline 4 & 1 male (12.5). & 24.ii.1891 (M24), Fukuji Sakamoto. & [Kanagawa Pref.], Miura, Misaki. & - \\
\hline 5 & 1 male $(12.5)$ & Sept., 1885 (M 18). & Tokyo market. & - \\
\hline
\end{tabular}

6. Sepia elliptica Hoyle, 1885. 'aka hari-ika' [red needle-squid] [Sepia madokai Adam, 1939] (Ikeda, 1891f: 328).

\begin{tabular}{cccc}
\hline lot\# & Specimens & Date and Collector & Locality \\
\hline $1^{*}$ & 1 male (5). & 14.ii.1891 (M 24), Fukuji Sakamoto. & Soshu [Kanagawa Pref.], Miura, Misaki. \\
\hline
\end{tabular}

7. Sepia sulcata Hoyle, 1885. 'aka hari-ika' (Ikeda, 1891f: 328-329).

\begin{tabular}{|c|c|c|c|c|}
\hline lot\# & Specimens & Date and Collector(s) & Locality & Comments \\
\hline $1 *$ & 5 males, 7 females. & Sept., 1885 (M 18). & Tokyo market. & ML $3.5-5.5 \mathrm{~cm}$ \\
\hline 2 & 3 females. & 14.ii.1891 (M 24), Fukuji Sakamoto. & Soshu [Kanagawa Pref.], Miura, Misaki. & Largest ML $6 \mathrm{~cm}$. \\
\hline
\end{tabular}


8. Sepia kiensis Hoyle, 1885. 'aka hari-ika' (Ikeda, 1891f: 329).

\begin{tabular}{|c|c|c|c|c|}
\hline lot\# & Specimens & Date & Locality & Comments \\
\hline 1 & 5 males, 11 females. & Sept., 1885 (M 18). & Tokyo market. & ML $4.0-5.5 \mathrm{~cm}$ \\
\hline 2 & 1 male (5), 1 female (3). & Feb., 1891 (M 24). & Soshu [Kanagawa Pref.], Miura, Misaki. & - \\
\hline
\end{tabular}

\section{Genus Sepiella.}

Sepiella maindroni sensu Hoyle, 1886. [Sepiella japonica Sasaki, 1929] (Ikeda, 1891f: 330).

\begin{tabular}{|c|c|c|c|c|}
\hline lot\# & Specimens & Date and Collector(s) & Locality & Comments \\
\hline $1 *$ & 2 females. & 8.ii.1891 (M 24), Fukuji Sakamoto. & $\begin{array}{l}\text { Soshu [Kanagawa Pref.], Natagiri } \\
\text { [Yokosuka City, Natsushima]. }\end{array}$ & Both ML $12.7 \mathrm{~cm}$. \\
\hline $2^{*}$ & 1 male, 3 females. & 1.x.1885 (M 18). & Tokyo market. & ML $4.0-7.5 \mathrm{~cm}$. \\
\hline
\end{tabular}

Incl. in synonymy: Sepia inermis Hasselt [in d'Orbigny], S. sinensis Gray, 1849, S. microcheirus Gray, 1849, S. affinis 'Sau.' and S. tourannensis 'Sau.' (S. affinis and S. tourannensis were described originally by Eydoux and Souleyet, 1852).

\section{Tetrabranchiata, Family Nautilidae, Genus Nautilus.}

Nautilus pompilius Linnaeus, 1758. (Ikeda, 1891f: 331).

\begin{tabular}{clll}
\hline lot\# & \multicolumn{1}{c}{ Specimens } & \multicolumn{1}{c}{ Date and Collectors } \\
\hline 1 & 1 spec. & April, 1884 (M 17), Mitsukuri and Ishikawa. & Locality \\
2 & 1 spec. & April, 1887 (M 20), Okada. & $\begin{array}{l}\text { Shunshu [Shizuoka Pref.], Enoura. } \\
\text { [Sagami Bay], Izu Shichito (Izu Shoto Islands), } \\
\text { Oshima. } \\
\text { Ryukyu [Okinawa], Yaeyama [islands near } \\
\text { Taiwan]. }\end{array}$ \\
& 1 spec. & (no data) & (no data) \\
\hline
\end{tabular}

Corrections and omissions (listed in Ikeda, 1891f: 331).

1. It is noted that the data on Loligo bleekeri were duplicated in Ikeda, 1891a: 30, and Ikeda, $1891 b: 60$ [though not exactly: for lot \#1, the ML listed is $24 \mathrm{~cm}$ in $1891 a$ but $34 \mathrm{~cm}$ in $1891 b$ ].

2. Three additional localities for Sepia esculenta from the literature are appended to Ikeda, 1891d: 242.

3. Another published locality for Sepia peterseni is appended to Ikeda, 1891e: 292. 